\title{
Piracy and P2P file-sharing networks : an economic analysis
}

Citation for published version (APA):

Yang, M. S. (2010). Piracy and P2P file-sharing networks : an economic analysis. [Doctoral Thesis, Maastricht University]. Datawyse / Universitaire Pers Maastricht. https://doi.org/10.26481/dis.20101124my

Document status and date:

Published: 01/01/2010

DOI:

10.26481/dis.20101124my

Document Version:

Publisher's PDF, also known as Version of record

\section{Please check the document version of this publication:}

- A submitted manuscript is the version of the article upon submission and before peer-review. There can be important differences between the submitted version and the official published version of record.

People interested in the research are advised to contact the author for the final version of the publication, or visit the DOI to the publisher's website.

- The final author version and the galley proof are versions of the publication after peer review.

- The final published version features the final layout of the paper including the volume, issue and page numbers.

Link to publication

\footnotetext{
General rights rights.

- You may freely distribute the URL identifying the publication in the public portal. please follow below link for the End User Agreement:

www.umlib.nl/taverne-license

Take down policy

If you believe that this document breaches copyright please contact us at:

repository@maastrichtuniversity.nl

providing details and we will investigate your claim.
}

Copyright and moral rights for the publications made accessible in the public portal are retained by the authors and/or other copyright owners and it is a condition of accessing publications that users recognise and abide by the legal requirements associated with these

- Users may download and print one copy of any publication from the public portal for the purpose of private study or research.

- You may not further distribute the material or use it for any profit-making activity or commercial gain

If the publication is distributed under the terms of Article $25 \mathrm{fa}$ of the Dutch Copyright Act, indicated by the "Taverne" license above, 


\title{
Piracy and P2P File-sharing Networks
}

\author{
An Economic Analysis
}


Piracy and P2P File-sharing Networks

(c) Copyright Michael Shuo Yang, Maastricht 2010

This book was typeset by the author using $\mathrm{AT}_{\mathrm{E}} \mathrm{X}$.

Cover design by Audrey Picard

Published by Universitaire Pers Maastricht

ISBN 9789052789972

Printed in the Netherlands by Datawyse 


\title{
Piracy and P2P File-sharing Networks
}

\author{
Proefschrift \\ ter verkrijging van de graad van doctor \\ aan de Universiteit Maastricht, \\ op gezag van Rector Magnificus, \\ Prof. dr. G.P.M.F. Mols, \\ volgens het besluit van het College van Decanen, \\ in het openbaar te verdedigen \\ op woensdag 24 november 2010 om 16:00 uur
}

door

\section{Michael Shuo Yang}

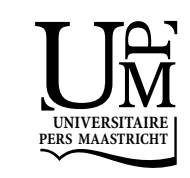


Promotor:

Prof. dr. P. J. J. Herings

\section{Copromotor:}

Dr. R. Peeters

\section{Beoordelingscommissie:}

Prof. dr. M. Carree (voorzitter)

Prof. dr. P. Belleflamme (Université catholique de Louvain)

Dr. I. Bos

Dit onderzoek werd financieel mogelijk gemaakt door Maastricht Research School of Economics of Technology and Organizations (METEOR). 


\section{Acknowledgements}

The front cover page of this book only bears my name, but the content inside benefited greatly from the invaluable contributions of many people around me during the course of my $\mathrm{PhD}$ project for more than 4 years. I therefore would like to take this opportunity to thank them for their help that has meant so much to me over the years.

First and foremost, the papers embedded in the chapters of this book would never have been up to their current standard without the direct involvement and supervision of my promoters Jean-Jacques and Ronald. Despite their busy schedules, they have always devoted ample time to meet with me and discuss the nitty-gritty details of the papers. I realize how lucky I am to have such great supervisors, and I undoubtedly have learnt a great deal from them. I cannot thank them enough for their top-notch supervision and outstanding support. I am also immensely grateful to the helpful comments the members of the assessment committee, to Ingrid and Mrs. Rohde for correcting the grammar in my Dutch Summary, to Elke, Nicole and Sylvia for their excellent administrative support, and to the METEOR research school for their financial support of this project. Furthermore, I would like to thank Ingrid and Arkadi for being my paranymphs.

People often say that doing a PhD can sometimes be quite a lonely endeavor. Luckily for me, with the great company of colleagues and friends around me, life outside working hours has in fact been a tremendous enjoyment. Among this lot, I must first thank Arkadi and Attila, who are chiefly responsible for making the past years so much fun. In the early 
years though, it was Rory who kept me good company when things started to look decidedly nerdy. During working hours, Ingrid always made sure that our office was a nice place to be where we shared our pain and joy of our $\mathrm{PhD}$ working lives. Then there was the Mensa gang with whom even the mediocre canteen food became pleasant dinners for working days, and of course the Wok gang with whom the Friday dinners were always such a nice thing to look forward to. The list of names would be too long, but I would like to thank all of these nice colleagues and friends who made my life so enjoyable.

Equally important as friends are the family. I would like to thank my parents for making all of this possible by sending me abroad to study regardless of costs, and the continuing encouragement and support when things weren't going particularly well. I am forever indebted to them. I would also like to thank my late grandmother who enlightened me and stimulated me for academic achievements from a very young age.

The person who deserves my utmost gratitude is Audrey, who has always been there, all these years, to encourage me and comfort me whenever I felt frustrated or lost. In fact, she also designed the front and back covers of this book. I dedicate this book to Audrey.

Michael Yang

Maastricht, November 2010 


\section{Contents}

$\begin{array}{ll}\text { Acknowledgements } & \text { i }\end{array}$

List of tables vii

$\begin{array}{ll}\text { List of figures } & \text { ix }\end{array}$

1 Introduction 1

1.1 P2P file-sharing networks $\ldots \ldots \ldots \ldots \ldots \ldots \ldots$

1.2 Network externalities . . . . . . . . . . . . . . . . 4

1.3 The platforms and the products $\ldots \ldots \ldots \ldots \ldots$

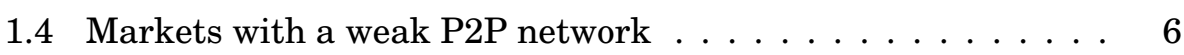

1.5 Markets with a strong P2P network $\ldots \ldots \ldots \ldots \ldots$

1.6 A dynamic point of view . . . . . . . . . . . . . . 10

2 Markets with a weak P2P network 13

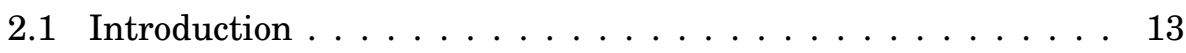

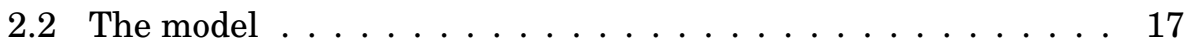

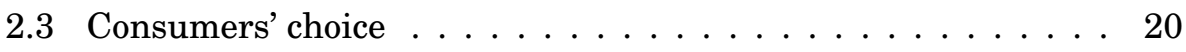

2.4 The firm's decision . . . . . . . . . . . . . . 27

2.4.1 Subgame-perfect Nash equilibria . . . . . . . . . . 27

2.4 .2 Equilibrium selection . . . . . . . . . . . . . . . 29

2.5 Comparative statics $\ldots \ldots \ldots \ldots \ldots \ldots$

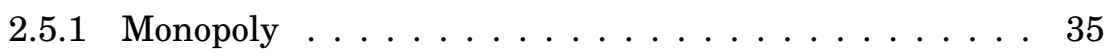

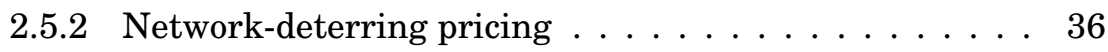




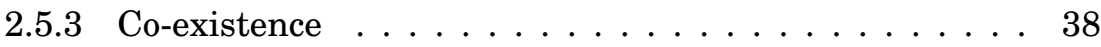

2.5.4 Comparative statics across market structures . . . . . 39

2.6 Discussion . . . . . . . . . . . . . . . . . . . . . 40

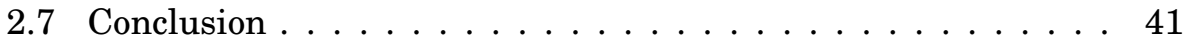

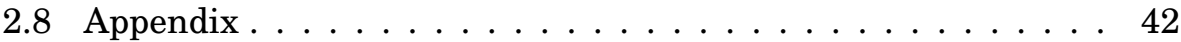

2.8.1 The fully-served multi-platformed market . . . . . . . 42

2.8.2 The partially-served multi-platformed market . . . . . 46

2.8.3 Support for Proposition 2.5.1-2.5.3 . . . . . . . . . . . . 47

3 Markets with a strong P2P network $\quad 53$

3.1 Introduction . . . . . . . . . . . . . . . 53

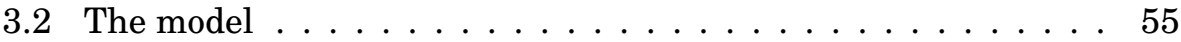

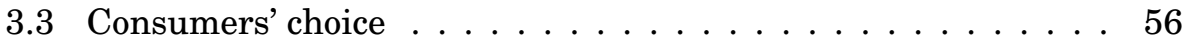

3.4 Firm's decision . . . . . . . . . . . . . . . . . . . 62

3.5 Equilibrium market structures $\ldots \ldots \ldots \ldots$. . . . . . 70

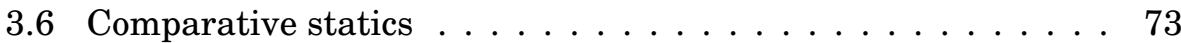

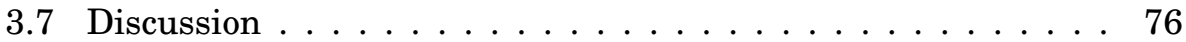

3.8 Concluding remarks $\ldots \ldots \ldots \ldots \ldots \ldots \ldots$

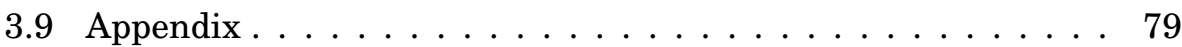

3.9.1 Solutions of the multi-platformed market structures . . 79

3.9.2 Supports of Proposition 3.3.1-3.3.3 . . . . . . . . . . . 81

4 A dynamic point of view $\quad 89$

4.1 Introduction . . . . . . . . . . . . . . . . . . . . 89

4.2 The model . . . . . . . . . . . . . . . . . . . . . 92

4.3 Numerical method . . . . . . . . . . . . . . . . . . . . 95

4.3.1 Discretization . . . . . . . . . . . . . . . . . 95

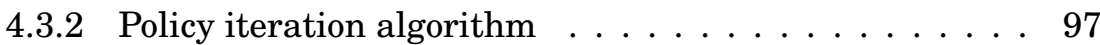

4.4 Model calibration and benchmark outcomes . . . . . . . . . . 99

4.4.1 Data and evidences . . . . . . . . . . . . . . . . . 99

4.4 .2 Parameter calibration . . . . . . . . . . . . . . . . . . 100

4.5 Model outcomes and analysis . . . . . . . . . . . . . . . . 102

4.6 Comparative statics . . . . . . . . . . . . . . . . 105

4.7 Discussion . . . . . . . . . . . . . . . . . . . 108

4.8 Concluding remarks . . . . . . . . . . . . . . . . . 110 
Contents

4.9 Appendix . . . . . . . . . . . . . . . . . . . . . . 112

4.9 .1 Tables . . . . . . . . . . . . . . . . . . . . . . 112

4.9 .2 Triangles . . . . . . . . . . . . . . . . . . . 113

$\begin{array}{ll}\text { Bibliography } & 119\end{array}$

Nederlandse samenvatting (Dutch summary) 123

$\begin{array}{ll}\text { Curriculum Vitae } & 129\end{array}$ 



\section{List of Tables}

2.1 All possible market structures in stage two. . . . . . . . . . 21

2.2 Three segments of equilibrium structures. . . . . . . . . . . 23

2.3 Possible scenarios and corresponding parameter settings. . . . 25

2.4 Monopoly pricing with fully-served market (left); Monopoly pricing with partially-served market (right). The cells display the signs of the first derivatives. . . . . . . . . . . . . . 35

2.5 Fully-served market (left); Partially-served market (right). The cells display the signs of the first derivatives. . . . . . . . . 36

2.6 Co-existence of the firm and the network with a fully-served market. The cells display the signs of the first derivatives. . . . 38

3.1 All possible market structures in stage two. . . . . . . . . . 57

3.2 Examples of parameter values for Proposition 3.4.1 (high heterogeneity scenario $\ldots \ldots \ldots \ldots$. . . . . . . . 6 65

3.3 Examples of parameter values for Proposition 3.4.2 (medium heterogeneity scenario) . . . . . . . . . . . . . 68

3.4 Examples of parameter values for Proposition 3.4.3 (low heterogeneity scenario) . . . . . . . . . . . . . . . . 69

3.5 Multi-platformed partially-served (local monopoly) market. . . 74

4.1 Calibrated parameter values. . . . . . . . . . . . . 100

4.2 Evolution of market outcomes over years. . . . . . . . . . . 104

4.3 Comparative statics of steady state outcomes without P2P. . 105 
4.4 Comparative statics of steady state outcomes with P2P. . . . 106

4.5 RIAA 2008 Year-End U.S. CD sales statistics. . . . . . . . . . . 112

4.6 Some predictions of market outcomes in the future. . . . . . . 112 


\section{List of Figures}

2.1 Fully-served market and partially-served market. . . . . . . . 21

2.2 scenario A.1 (left); scenario B.1 (right). . . . . . . . . . . . 23

2.3 Parameter settings leading to each of the scenarios. . . . . . . 25

2.4 Profit along segments; scenario A.1 (left); scenario B.1 (right). . 27

2.5 The lower envelope of the different scenarios (left: A scenarios; right: B scenarios). . . . . . . . . . . . . . . . . 28

2.6 A graphical illustration of the possible subgame-perfect Nash equilibrium market structures. . . . . . . . . . . . 33

2.7 Equilibrium price (left), profit (middle), and welfare (right) across markets structures. . . . . . . . . . . . . . . 39

3.1 Fully-served market and partially-served market. . . . . . . . 57

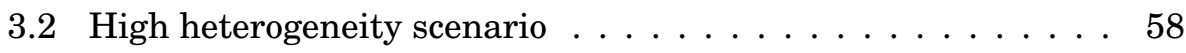

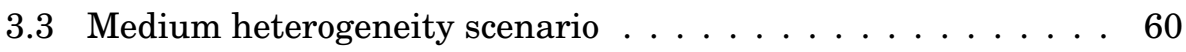

3.4 Low heterogeneity scenario. . . . . . . . . . . . . . 61

3.5 The lower envelop of the low heterogeneity scenario. . . . . . 63

3.6 Profit functions and equilibrium outcomes (high heterogeneity scenario $\ldots \ldots \ldots \ldots \ldots \ldots \ldots$

3.7 Profit functions and equilibrium outcomes (medium heterogeneity scenario) . . . . . . . . . . . . . 68

3.8 Profit functions and equilibrium outcomes (low heterogeneity scenario $\ldots \ldots \ldots \ldots \ldots \ldots \ldots$. . . . . . . . 70 
4.1 An illustration of the discretized state space and the discretized transition mapping. . . . . . . . . . . . . . . . 96

4.2 Stationary optimal pricing policy before P2P. . . . . . . . . . 113

4.3 Stationary optimal pricing policy with P2P. . . . . . . . . . . 114

4.4 Probability distribution over states in steady state just before the introduction of P2P (1999). . . . . . . . . . . . . . . 114

4.5 Probability distribution over states one year after the introduction of P2P (2000). . . . . . . . . . . . . . . . 115

4.6 Probability distribution over states two years after the introduction of P2P (2001). . . . . . . . . . . . . . . . . . . 115

4.7 Probability distribution over states three years after the introduction of P2P (2002) . . . . . . . . . . . . . . . . . 116

4.8 Probability distribution over states four years after the introduction of P2P (2003). . . . . . . . . . . . . . . . . . . 116

4.9 Probability distribution over states nine years after the introduction of P2P (2008). . . . . . . . . . . . . . . . 117

4.10 Probability distribution over states in steady state after the introduction of P2P (approximately 2020). . . . . . . . . . . 117 


\title{
Chapter 1
}

\section{Introduction}

\author{
"You wouldn't steal a car! \\ You wouldn't steal a handbag! \\ You wouldn't steal a television! \\ Downloading pirated films is stealing! \\ Piracy. It's a crime!"
}

Or is it really? You see, if you steal a car from someone, that person loses the car. The same goes for the handbag and the television. But if you download a film from the internet for free, is it still so clear who loses what? Economists call this the non-rival nature of a good, meaning that one person's consumption of it does not prevent simultaneous consumption by other consumers. But what about the forgone sales revenue of the producer, you ask? That would be extremely hard to quantify if this is a film that you would never have bought anyway. Indeed, the economic and social impact of the piracy of digital goods (such as CDs, DVDs, games and software) are far from as straightforward as suggested in the above message from the RIAA.

This thesis addresses some of the complex issues associated with the online piracy of digital goods in the first decade of the 21 st century. We look at the strategic behavior of the monopolistic producer of digital goods, facing the threat of online piracy. "How does the firm price its products optimally facing the demand and the competition from piracy?" We study the economic impacts of online piracy on consumers as well as producers. "Who are the

\footnotetext{
${ }^{1}$ Recording Industry Association of America (RIAA) anti-piracy advertisement.
} 
winners and the losers, and by how much?" "What determines that?" We analyze the impacts of the enforcement of intellectual property rights law. "Does pursuing and punishing downloaders enhance social welfare?" We investigate various conditions for the market of digital goods to exhibit certain characteristics. "When is the market served by a single or multiple active platforms and when is the market fully or partially served?" Finally, we observe the dynamic development of the battle between the digital goods producer and the pirating platform, from its infancy to its full-scale establishment, and its impact on the consumers, the producer and the society as a whole.

\subsection{P2P file-sharing networks}

Piracy has existed ever since there were intellectual properties, chiefly in the form of making and distributing of unauthorized copies in black markets. Unlike the black-market piracy which dominated the previous century, today's online piracy is mainly fostered, among other things, by a technology known as the peer-to-peer (P2P) file-sharing networks. ${ }^{2}$

P2P file-sharing networks have been around for more than 10 years. The earliest form of this technology came in existence at the turn of the century in the form of Napster, and was immediately welcomed with open arms by the internet community. Just as was warmly receipted by the users, was it furiously resented by the publishing firms. By the time it was forced by the authorities to shut down in 2001, it had gained up to around 8 million users world-wide in just two years.

The rise and the fall of Napster engendered a generation of P2P networks in various forms and sizes, Kazaa, Gnutella, FastTrack, Limewire and Edonkey, just to name a few. None of them has reached the scale of Napster in terms of market share, 78\%, according to a study done by (Ghosemajumder 2002), but together they represent a growing P2P user population. For many years now, P2P activities have been accounting for more than half of all internet traffic. ${ }^{3}$ Over the years, some P2P networks have thrived and others

\footnotetext{
${ }^{2}$ Some non-P2P file-sharing (hosting) sites such as RapidShare, have also gained tremendous popularity towards the end of the $00 \mathrm{~s}$.

${ }^{3}$ According to a study conducted by the CacheLogic, a network solution company, in 2004,
} 
perished, amid the vigourous legal attacks initiated by publishing firms and regulatory bodies alike. For instance, Kazaa, Edonkey and P2P search engines such as ShareReactor and Piratebay have all come under legal attack at some point in time. Furthermore, charges have been pressed against individual "front runners" of P2P users. In 2005, a criminal court in the Paris suburb of Pontoise fined a teacher about $€ 10,000$ in damages for counterfeiting after he was found guilty of downloading nearly 10,000 tracks, according to the Washington Post. ${ }^{4}$ The same source has revealed that in the U.K. and the U.S. industry groups and recording companies have filed thousands of law suites against individuals who allegedly shared music.

In order to survive the legal battles initiated by the publishing firms, the evolution of the P2P technology has gone through several phases, each more efficient and more difficult to track down than its predecessor. The latest incarnation that accounts for the majority of $\mathrm{P} 2 \mathrm{P}$ traffic today is known as BitTorrent. $^{5}$

We will take the BitTorrent protocol as an example to explain the working principles of modern peer-to-peer file sharing. Suppose a user wants to share a music file that he has on his computer with other users. In this case he is called a seeder. The seeder makes the music file - called the seed available for other users, known as peers, by creating a "torrent" file which contains metadata about the seed and the tracker - the server that coordinates the distribution of the seed. When new peers join and request the music file from the seeder, they only have to download the "torrent" file (which is a tiny file). After that, the BitTorrent software on the peers' computers will contact the tracker and start downloading pieces of the seed from the seeder. However, different peers receive different pieces of the seed. Once multiple peers join to download the seed from the seeder, the BitTorrent software allows peers to download different missing pieces of the seed from each other without having to download them from the seeder directly. This procedure, therefore, triggers an exponential increase in the downloading speed

P2P traffic makes up 55\% of the total internet traffic in Europe and $80 \%$ in Asia.

${ }^{4}$ French Criticize Music Download Crackdown, The Associated Press, The Washington Post, 2005.

${ }^{5}$ Technically speaking, BitTorrent is a P2P file-sharing protocol, not a real P2P network. But for the purpose of our analysis, it makes absolutely no difference. 
without unnecessarily straining the bandwidth of the seeder. When a peer successfully completes the downloading of the music file from the seeder, she will automatically become a new seeder herself. The more seeders there are, the "healthier" the shared file becomes. The tracker stores all the information about the current seeders and peers. Thus, as soon as there are more than one seeder seeding the file, the original seeder can safely go offline, knowing that the file will continue to be shared within the network.

\subsection{Network externalities}

The above phenomenon where the presence of an extra peer in the network adds to the time-efficiency of the downloading process can be classified as a form of positive direct network externalities. This effect is present in all network products such as telephone networks, fax, or even social networking websites like Facebook, where the value of a product is basically determined by the number of users in the network. An additional user, therefore, directly adds value to the product. Consider, on the other hand, a computer operating system such as Windows. The value of such an operating system is not directly determined by how many people are using it. But the more people who adopt this operating system, the more applications will be written for it by software developers due to the potential profits. This, in turn, makes the operating system itself more attractive. An extra user, therefore, adds indirectly to the value of the product. This is often referred to as a form of indirect consumptive externalities.

Although indirect consumptive externalities do exist for some products shared on the P2P network, such as software, they do not have such a profound effect on other products like music and movies. On the other hand, the direct network externalities of the P2P network exist for all products shared on the network, and therefore will constitute the building rationale for all the chapters in this thesis.

Some careful modeling of the network externalities is vital. We observe that the externalities are monotonic, i.e. an additional user always adds value to the network by potentially reducing downloading time. Notice the difference between $\mathrm{P} 2 \mathrm{P}$ and non-P2P file sharing in the sense that $\mathrm{P} 2 \mathrm{P}$ sharing is not plagued with the usual network caveat of traffic congestions. Gen- 
erally, the more peers there are who are downloading the same file, the faster it is for everyone to download it. In the literature of economics of network products, this effect is mostly modeled as linearly increasing in the number of users. ${ }^{6}$ However, we observe that in reality this effect is far from linear. An additional peer's marginal contribution to the reduction of downloading costs diminishes as the number of peers increases. In order to model this, we construct a function of downloading costs (in terms of downloading time) that is decreasing and convex in the number of peers. We believe that this is the best way to realistically reflect the working principles of P2P networks as they are.

\subsection{The platforms and the products}

In this thesis, we consider two platforms: one is a content provider which will be called the "firm" throughout the thesis; and the other is a P2P network. The firm is offering an original version of an information content product, for example a music CD or a DVD movie, for a price at which consumers can buy. The product is then "ripped" by a consumer and subsequently uploaded onto the P2P network for users to download free of charge, in the spirit of "sharing". We will refer to the first product as the physical form of the product and the latter the digital form.

The consumers can choose either one form of the two if they so wish, depending on their personal tastes and the associated costs. The cost of the physical form is obviously the price that is charged by the firm, and the cost of the digital form is the waiting time that is required before it is downloaded via the P2P network. Naturally, no one is forced to consume either form. As a result, some consumers may end up being unserved, leading to a partiallyserved market structure. Because consumers always prefer one form to the other, they will not choose to consume both forms, which is referred to as "multi-homing" in the literature when there are two fundamentally different products.

Out of the two platforms, only one is a profit-maximizing agent, namely, the firm. The P2P network itself is non-proprietary, and seeks no profit max-

\footnotetext{
${ }^{6}$ See, for instance, (Gayer and Shy 2003).
} 
imization. It is essentially an out-side market phenomenon, but one that has a significant impact on the strategic behaviors of the active parties inside the market, which ultimately affects the market structure and performance.

\subsection{Markets with a weak P2P network}

A P2P network gathers its momentum once a reasonable amount of users join. Like any network product, the size of the network determines its strength. The network size is treated as an endogenous variable, since it is the consumers that decide whether or not to join a certain network. However, there are exogenous factors that determine the inherent strength of a network. Think, for instance, about the degree of intellectual property rights protection. In a country where illegal file sharing is strictly prohibited, the P2P networks will have a tough time gathering users. Another exogenous factor equally important is the quality of the digital form of the product as a result of the prevailing technology of digital copying. In a market with low quality digital copies, the $\mathrm{P} 2 \mathrm{P}$ networks will have less potential users than in a market with high quality digital copies.

Chapter 2 is an adapted version of the paper (Herings, Peeters, and Yang 2010), which considers markets with an inherently weak P2P network. This is manifested by a parameter assumption that when a network is empty, no one will join. Hence, the network is vulnerable against a coordination failure. In particular, if all consumers expect the network not to form, it will be individually rational for every consumer not to join the network, despite the fact that some of them would have been better off had they all joined the network together. In reality, we observe weak P2P networks when there is a lack of enthusiastic users that selflessly provide the availability of the files being shared.

We model the interaction of the firm and the P2P network as a two-stage game. In the first stage, the firm sets a price for the physical form of the product. Then, in the second stage, after having observed the price set by the firm, consumers simultaneously and independently decide whether to legally buy the physical form, to download the digital form via the P2P network, or not to acquire the product at all. The consumers are heterogeneous with respect to their tastes regarding the physical and the digital form, and 
we use the standard Hotelling model to describe their preferences. In their decisions, consumers have to anticipate each others' decisions, since the resulting network size determines the actual costs of downloading. We analyze the model by studying its subgame-perfect Nash equilibria.

In the second stage of our game, consumers face the coordination problem of network forming. If they all expect that no one joins the P2P network, then it is optimal for all of them not to join. In this case, there is a critical mass (Economides 1996) such that a P2P network of that size can be supported as an equilibrium, where there is a marginal consumer that is indifferent between buying the physical form and downloading the digital form. Due to the positive downloading externalities, this critical-mass network is destabilized when slightly more or slightly less consumers join it. When slightly more consumers join the network, more and more consumers will want to join as well, to a size where a new marginal consumer is indifferent between buying and downloading, or where the network contains all consumers and everybody is better off downloading. Conversely, When slightly less consumers join, that is, if the critical mass of the network is not reached, then less and less consumers will want to stay in the network, resulting in a totally empty network in the end. Note that this is only an interpretation of the network forming process. We do not really model the process dynamically, but focus instead on the static nature of the problem which is analytically more tractable.

In chapter 2, we provide a full characterization of the subgame-perfect Nash equilibria of the model and then restrict our attention to the unique subgame-perfect Nash equilibrium where the network of maximal size forms whenever possible. We characterize the equilibrium market structures, and analyze for each structure how the firm's profit, the consumers' surplus and the total welfare are affected by different parameters such as the generic cost factor of downloading and the consumers' taste heterogeneity.

We find that there are three possible equilibrium market structures. Firstly, the firm may act as a monopoly, either partially or a fully serving the market, depending on the circumstances. In this case, the P2P network does not form. For this to occur, the intrinsic value of the physical form of the product must be substantially higher than that of the digital form. Secondly, the firm may prevent the forming of the network by means of a 
network-deterring pricing policy. Again, the firm either partially or fully serves the market. Finally, the firm and the P2P network may co-exist. In this case, the market is guaranteed to be fully served.

In the comparative statics of the chapter, we find that the larger the generic cost factor of downloading is, the less likely a P2P network will form, and the higher the firm's profit will be. Strikingly, when the two platforms co-exist, the smaller the cost factor is, the higher the price set by the firm. This is at first glance counter-intuitive. But it is in fact a very interesting form of platform competition. Recall that downloading costs decrease with the network size at a diminishing rate. This makes the first few consumers who join the network more vital in determining the costs of downloading. The firm, therefore, has an incentive to "play tough" by means of a low price when the equilibrium network size is relatively small (inherently weak). Conversely, if the cost factor is small, the equilibrium network size is large, and the network is stronger and will form more easily. In that case, the firm can do better by backing off from the competition and charging a high price to reap the most profit out of the customers that are more eager to buy the physical form of the product. This can be seen by the equilibrium profit being increasing in the cost factor, despite the decreasing price.

In both the partially-served monopolistic market with network-deterring pricing and the fully-served market with multi-platform co-existence, the total welfare decreases in the cost factor. This implies that it is welfare decreasing if authorities overemphasize the protection of intellectual property rights.

\subsection{Markets with a strong P2P network}

Chapter 3 is an adapted version of the paper (Yang 2010), which studies markets with an inherently strong P2P network. This is manifested by a parameter assumption that there exists at least one consumer who will join the P2P network even when the network is expected to be empty. The focus here is to capture the case where there are always some fanatic $\mathrm{P} 2 \mathrm{P}$ supporters who keep the network alive by seeding. This can happen, for instance, in a country where illegal file sharing is largely tolerated, where the P2P networks will gathering users easily. It can also happen in a market with 
high quality digital form of the product, where the P2P networks will have more potential users than in a market with low quality digital copies.

The model is taken from chapter 2 , with the above change in parameter settings. Moreover, we relax the assumption on the relatively small parameter value of consumers' taste heterogeneity. More specifically, we now allow consumers' taste heterogeneity to be so high that consumers with extreme preferences may not consume the least preferred version of the product even if it is costless. In so doing, we are able to capture more scenarios, including those where the market is partially served by the firm and the network together.

We discover that coordination failure in the forming of networks is not an issue once we consider a high level of taste heterogeneity and include such fanatic users in the model. Note that under the settings of this chapter, in the absence of the firm, the network will always exist. In other words, the consumers who prefer the digital form will always be served. In contrast, under the settings of Chapter 2, even in the absence of the firm, the network may not exist if the consumers fail to coordinate due to pessimistic expectations.

In total, we find five possible equilibrium market structures in this chapter. Out of the five market structures, the most prevalent is the one where the firm accommodates the network and competes in price. In this case, the firm's profit suffers and the total welfare benefits from the existence of P2P.

The firm can still deter the network, but under the circumstances where the taste heterogeneity is low, the quality of the physical form is high, and the legal enforcement is relatively strict. The firm may also want to serve the whole market, if the value of its product is significantly higher than that of the pirated version, and taste heterogeneity is low. Another special market structure is that of a market-sharing case, which happens when taste heterogeneity is just so that the firm chooses to precisely serve the consumers who prefer not to download. Effectively, the firm lets the network form to its maximum size, and then serves the residual demand. Thus, the firm and the network together serve the market fully without competing with each other. Furthermore, there is a possibility where the firm can ignore the network and act as a monopoly, when taste heterogeneity is huge. Yet in that case the firm's profit actually decreases in taste heterogeneity. So does the con- 
sumers' surplus. Total welfare, therefore, decreases in taste heterogeneity, and in the cost factor of downloading. This result is in accordance with the findings in chapter 2 .

\subsection{A dynamic point of view}

The analysis in chapter 2 and 3 are static in nature. This implies that in the presence of demand-side externalities there may be multiple equilibria, which ultimately leads to an equilibrium selection of some sort. In particular, we always focused on the maximally achievable network size. This type of analysis lacks insights on how such networks actually form, step by step, from zero to a steady-state network size; how the firm prices strategically to compete with the network every step of the way; and how society is affected during the process.

Only a dynamic model can bring such insights, which is what chapter 4 is designed to achieve. Dynamic stochastic models with network externalities have been recently studied in the IO literature, mainly using numerical methods. Chapter 4 is, therefore, an adapted version of the paper (Herings, Peeters, and Yang 2009), which adopts a dynamic stochastic model to solve for the optimal pricing policy of the music recording companies in the presence of P2P file-sharing networks eroding their CD sales.

In the model, the firm sets the price of its product every period and a continuum of consumers decide whether to purchase it, to download the digital form via the $\mathrm{P} 2 \mathrm{P}$ network, or not to acquire the product at all. The timing involves discrete periods with an infinite horizon. The firm is forward looking and strives to maximize the present value of all future profits by choosing a state-dependent pricing policy; the state being the market shares of the firm, of the P2P network, and of the unserved market. We employ a policy iteration algorithm on a discretized state space to numerically compute the optimal pricing policy. The consumers make one of the three choices based on the price and the state in order to maximize their utility. Consumers are ex ante identical, but receive random utility shocks prior to their purchasing decision every period anew. Switching costs are imposed on the consumers who switch to a product, from previously consuming the other product or not consuming at all. The parameters of the model have been calibrated in such 
a way that the model outcomes (such as prices and sales quantities) match the real-world data in the years 1999 and 2003.

An advantage of this approach is that we can study how networks develop in a dynamic process without having to make any assumptions on consumer coordination as in the conventional static models in chapter 2 and 3. We are no longer confronted with the issue of the critical mass of the network. We can also observe exactly how the firm sets its price conditioned on its market share and that of the network. Sometimes it sets the price very low in order to win vital market shares to fight against the P2P network, while other times accommodates the network by setting a high price to reap the profit from its own installed customer base.

Beside using the model to describe what might have happened in the CD market year on year since 1999 when P2P was introduced, we can also use the model to predict future sales quantities and to compute the changes in consumers' surplus and total welfare. For instance, the model shows that the sales quantity of the year 2008 should be 374.3 million copies, while the real figure is 384.7 million. It also predicts that the CD sales will have dropped to around 231.2 million copies per year around the year 2020, when according to the model estimations the long-run steady state is approximately reached. More importantly, with this model one can estimate the welfare gain over the years brought by the P2P file-sharing networks. In the year 2003, four years after P2P was introduced, while the music industry was suffering from a $\$ 1.6$ billion forgone profit per year, the total welfare was up by $\$ 13.1$ billion per year. By the year 2008, the industry profit has dropped by a further $\$ 1.4$ billion, and welfare soared by a further $\$ 12.4$ billion per year, making it $\$ 25.6$ billion more than in 1999. In the long-run steady state (around 2020 ), the industry profit will have dropped to a mere $\$ 865.5$ million per year, and total welfare are set to have improved since 1999 by a whopping $\$ 29.7$ billion per year. We are acutely aware that these estimates will be far from accurate, since the market conditions for such a ever-changing industry are impossible to predict for such a long time span. But this exercise gives a rough indication of what would happen in the future if everything were to stay as they are from now until then.

In the comparative static analysis of the model, one of our most important findings is that total welfare is negatively related to the generic cost 
factor of downloading. This result coincides with the findings from papers such as (Rob and Waldfogel 2006) and also our chapter 2 and 3, in the sense that the existence of $\mathrm{P} 2 \mathrm{P}$ actually enhances total welfare. This implies that by enforcing strict intellectual property rights law and making file-sharing more difficult for the consumers, the government is effectively curbing the society from enjoying a high level of welfare. 


\section{Chapter 2}

\section{Markets with a weak P2P network}

\subsection{Introduction}

This chapter studies the market of information goods with the presence of an inherently weak P2P (peer-to-peer) file-sharing networks. ${ }^{1}$ By a weak network, we mean that the network is vulnerable against a coordination failure. In particular, if all consumers expect the network not to form, it will be individually rational for every consumer not to join the network, despite the fact that some of them would have been better off had they all joined the network together. In reality, we observe weak P2P networks when there is a lack of enthusiastic users that selflessly provide the availability of the files being shared.

There are exogenous factors that determine the inherent strength of a network. Think, for instance, about the degree of intellectual property rights protection. In a country where illegal file sharing is strictly prohibited, the $\mathrm{P} 2 \mathrm{P}$ networks will have a tough time gathering users. Another exogenous factor equally important is the quality of the digital form of the product as a result of the prevailing technology of digital copying. In a market with low quality digital copies, the P2P networks will have less potential users than in a market with high quality digital copies.

\footnotetext{
${ }^{1}$ This chapter is an adapted version of the paper (Herings, Peeters, and Yang 2010).
} 
The classical literature related to piracy can be traced back to a couple of decades ago. Typical papers include for example (Novos and Waldman 1984), (Liebowitz 1985) and (Johnson 1985), where the authors study how a firm can react to piracy. Basically there are three possibilities, do not react at all if piracy poses no real threat, use piracy-deterring pricing, or use piracyaccommodating pricing. For a systematic analysis of the short- and long-run consequences of illicit copying of information goods, we refer to (Belleflamme 2003).

In parallel, the literature on network externalities has been developed, represented by classical papers including e.g. (Katz and Shapiro 1985), (Katz and Shapiro 1986), (Farrell and Saloner 1985) and (Farrell and Saloner 1986). (Conner and Rumelt 1991) and (Takeyama 1994) combine the two subjects and find that unauthorized reproduction of intellectual property in the presence of consumptive externalities can induce greater firm profits and lead to Pareto improvements.

More recently, since the rise and fall of the first generation peer-to-peer technology Napster and the controversy that ensued, a wave of new papers emerged to specifically tackle the P2P phenomenon. (Gayer and Shy 2003) studies how publishers of digitally-stored products can utilize P2P networks to enhance sales of their products sold in stores. This result follows from the positive consumptive externalities in their model. Another way in which firms can benefit from $\mathrm{P} 2 \mathrm{P}$ activities is in set-ups with imperfect information about product characteristics. The effects of sampling possibilities due to P2P networks are studied by (Peitz and Waelbroeck 2006b). The authors show that under sufficient taste heterogeneity and product diversity, the positive effect of downloading on sales due to sampling may compensate the negative effect. (Peitz and Waelbroeck 2006a) provides a comprehensive review of the recent literature on piracy of digital products.

While consumptive externalities are very important for software products, for the majority of illegal content shared on P2P networks (music, movies, etc.), this effect plays a role of lesser importance. We abstract from consumptive externalities, but instead focus on the network externalities caused by the working principle of the P2P file-sharing network itself. A higher number of users of a network improves the availability of files and hence decreases the standard search costs and downloading time. It is pre- 
cisely this network effect that we focus on in this study. This effect is not linear. The marginal benefit of an additional user joining the network is positive, but decreasing in the network size. Therefore, we implement a cost function of downloading that is decreasing and convex in the number of users of the P2P network.

Apart from the network size, the cost function of downloading is influenced by a parameter called the generic cost factor of downloading. It represents a collection of factors that may affect downloading costs, for instance, population computer literacy, the availability of broadband internet infrastructure, and most importantly, the degree of legal enforcement of intellectual property rights. Observe that unlike (Gayer and Shy 2003), legal buyers of the product do not benefit from the number of downloading users, which distinguishes our downloading externalities from consumptive externalities. We also abstract from other factors that tend to benefit the firm selling the product like imperfect information of product characteristics, or the possibility of indirect appropriation of rents (Liebowitz 1985). This eliminates channels through which the firm can benefit from piracy, and it is a priori unclear how total surplus is affected by piracy.

We consider a monopolistic firm that is confronted with an illegal P2P network. We refer to the legal product sold by the firm as the physical form and the $\mathrm{P} 2 \mathrm{P}$ version of it as the digital form. These two forms, although essentially providing similar contents, differ in numerous ways. The physical form of the product is sold by the firm as CDs or DVDs, usually accompanied by appealing packaging and complementary booklets. The digital form, which is shared on the internet via P2P networks, contains largely the same content, although often of discounted quality. It does not have the nice complementary features that the physical form does, and the quality of the content is often inferior.

We model the interaction of the firm and the P2P network as a two-stage game. In the first stage, the firm sets a price for the physical form of the product. Then, in the second stage, after having observed the price set by the firm, consumers simultaneously and independently decide whether to legally buy the physical form, to download the digital form via the P2P network, or not to acquire the product at all. The consumers are heterogeneous with respect to their tastes regarding the physical and the digital form, and 
we use the standard Hotelling model to describe their preferences. In their decisions, consumers have to anticipate each others' decisions, since the resulting network size determines the actual costs of downloading. This distinguishes our model from the literature on copying (Belleflamme 2003), where network effects are absent and decisions of other consumers are irrelevant. We analyze the model by studying its subgame-perfect Nash equilibria.

In the second stage of our game, consumers face a classical coordination problem. If they all expect that no one joins the P2P network, then it is optimal for all of them to buy the product legally from the firm. When the price set by the firm is sufficiently high, there is a critical mass (Economides 1996) such that a P2P network of that size can be supported as an equilibrium, where there is a marginal consumer that is indifferent between buying the physical form and downloading the digital form. Due to the positive downloading externalities, this critical-mass network is destabilized when slightly more consumers join it. Then, more and more consumers join the network up to a size where a new marginal consumer is indifferent between buying and downloading, or where the network contains all consumers and everybody is better off downloading.

We provide a full characterization of the subgame-perfect Nash equilibria of the model and then restrict our attention to the unique subgameperfect Nash equilibrium where the network of maximum size forms whenever possible. We characterize the equilibrium market structures, and analyze for each structure how the firm's profit, the consumers' surplus and the total welfare are affected by different parameters such as the degree of intellectual property law enforcement and the consumers' taste heterogeneity.

We find that there are three possible equilibrium market structures. Firstly, the firm may act as a traditional monopoly, either with a partiallyserved or a fully-served market. In this case, the P2P network does not form. For this to occur, the intrinsic value of the physical form of the product has to be substantially higher than that of the digital form. Secondly, the firm may prevent the forming of the network by means of a network-deterring pricing policy. Again, the firm either partially or fully serves the market. Finally, the firm and the P2P network may co-exist. In this case, the market is guaranteed to be fully served.

Regarding the effect of the generic cost factor of downloading, we find 
that the larger this cost factor is, the less likely a P2P network will form, and the higher the firm's profit will be. Strikingly, once the market exhibits coexistence of the two platforms, the smaller the cost factor is, the higher the price set by the firm. Despite this pricing behavior, the firm's profit unambiguously declines as the cost factor decreases. In the partially-served monopolistic market with network-deterring pricing and the fully-served market with multi-platform co-existence, the total welfare decreases in the cost factor. This implies that it is welfare decreasing if authorities overemphasize the protection of intellectual property rights for those contents whose creators are not significantly hurt by consumers' free-riding.

The remainder of this chapter is organized as follows. In Section 2.2 the two-stage model is described in detail. Next, the consumers' choices in the second stage, given the price set by the firm in the first stage, are presented in Section 2.3. Subsequently, in Section 2.4, the firm's pricing decision in the first stage, and the resulting market structures, are analyzed. In order to reduce multiplicity of equilibria, we restrict our attention to the case with coordination on the network of maximal size. The comparative statics of this equilibrium with respect to the model's parameters are provided in Section 2.5. Section 2.6 and 2.7 conclude and discuss the results.

\subsection{The model}

We consider a monopolistic firm offering an information good and facing a $\mathrm{P} 2 \mathrm{P}$ network. We model this situation by means of a two-stage game. In stage one, the firm sets a price $p$ for the physical form of the product. Next, in stage two, after having observed the price set by the firm, consumers decide simultaneously and independently whether to purchase the physical form sold by the firm $(S)$, to download the digital form via the P2P network $(N)$, or not to acquire the product at all $(\varnothing)$. We assume that there is a continuum of consumers with the mass of 1 who differ in their relative preferences of the physical form over the digital form. ${ }^{2}$ A strategy of a consumer with identity $x \in[0,1]$ is a function $d^{x}$ that maps any price $p$ into her choice

\footnotetext{
${ }^{2}$ Note that the size of the total market is therefore implicitly assumed to be 1 .
} 
set:

$$
d^{x}: \mathbb{R}_{+} \rightarrow\{S, N, \varnothing\}
$$

and we denote the profile of strategies by $d=\left(d^{x}\right)_{x \in[0,1]}$.

The price $p$ set by the firm and the profile of consumers' strategies determine the sales $s(p, d)$ by the firm and the size $n(p, d)$ of the network via

$$
s(p, d)=\mu\left(\left\{x \in[0,1] \mid d^{x}(p)=S\right\}\right) \text { and } n(p, d)=\mu\left(\left\{x \in[0,1] \mid d^{x}(p)=N\right\}\right),
$$

where $\mu$ denotes the Lebesgue measure. ${ }^{3}$

We assume, for simplicity, that the firm has zero costs in production and aims to maximize its profit, given by

$$
\pi(p, d)=p \cdot s(p, d)
$$

The utility of a consumer with identity $x \in[0,1]$ is given by

$$
U^{x}(p, d)= \begin{cases}\beta-\tau x-p & \text { if } d^{x}(p)=S \\ \gamma-\tau(1-x)-C(n(p, d))) & \text { if } d^{x}(p)=N \\ 0 & \text { if } d^{x}(p)=\varnothing\end{cases}
$$

where $\beta>0$ and $\gamma>0$ represent the basic utility of the physical and the digital form respectively. The identity $x \in[0,1]$ reflects the consumer's relative preference over the two forms. The consumer with identity $x=0$ has a strong preference for the physical form, whereas the consumer with identity $x=1$ has a strong preference for the digital form. For consumers $x \in(0,1)$, the acquisition of one of the forms generates a disutility that depends on the identity $x$ and the parameter $\tau>0$. The parameter $\tau$ captures the amount of heterogeneity in consumers' tastes. Finally, $C(n)$ represents the costs of downloading when the resulting network is of size $n \in[0,1]$.

We focus on the network externalities caused by the working principle of the P2P file-sharing network itself. A higher number of users of a network improves the availability of files and hence decreases the standard search costs and downloading time. The marginal benefit of an additional user joining the network is positive, but decreasing in the network size. Therefore,

\footnotetext{
${ }^{3}$ In equilibrium, the sets of consumers buying from the firm and going to the network, respectively, will be measurable.
} 
we implement a cost function of downloading that is decreasing and convex in the number of users of the P2P network, ${ }^{4}$

$$
C(n)=\sigma \cdot(1-n)^{2},
$$

where $\sigma>0$ represents the generic cost factor of downloading, incorporating a collection of factors that may affect downloading costs, for instance, population computer literacy, the availability of broadband internet infrastructure, and most importantly, the degree of legal enforcement of intellectual property rights. Note that $\sigma$ is identical for every consumer and is independent of the network size.

We impose some assumptions on the relevant parameters.

Assumption 2.2.1. $\beta>\gamma$.

Personal preferences aside, the objective product quality of the original physical form is higher than that of the digital form (which is "ripped" from the original).

Assumption 2.2.2. $\beta>\tau$ and $\gamma>\tau$.

Every consumer prefers a free physical product and a costless download to not acquiring the product.

Assumption 2.2.3. $\sigma>\gamma$.

The generic cost factor of downloading is sufficiently high such that when the network size is zero, no consumer would like to join the network.

Although irrelevant for the economic implications resulting from the model, we impose some assumptions on the consumers' behavior in case of indifference. When a consumer is indifferent between buying from the firm and not acquiring the product or between the firm and the network, she chooses the firm. When she is indifferent between the network and not acquiring the product, she chooses the network. Thus, without loss of generality, we assume a linear order of priority of the firm $(S)$ above the network $(N)$, and the network $(N)$ above not acquiring the product $(\varnothing)$.

\footnotetext{
${ }^{4}$ The quadratic specification of the cost function for downloading satisfies the qualitative properties just stated, while it preserves analytical tractability of the model.
} 
Finally, we restrict our attention to the case where the firm's price is bounded from above by the maximum that a consumer is willing to pay for the product; i.e., $0 \leq p \leq \beta$. If $p>\beta$, all consumers would have negative utility level from buying from the firm, hence the firm's sales and profit would be zero. The firm can always do better, in equilibrium, by lowering the price to the interval $[0, \beta]$. Hence, this assumption can be made without loss of generality and does not impose any restrictions on the results.

In the following sections we present the subgame-perfect Nash equilibria of the model. As usual, we start the analysis with determining the possible Nash equilibria for each of the subgames. That is, we determine the Nash equilibria of the games in the second stage that result from each possible first-stage price. Then, we consider the firm's pricing behavior in the first stage and find the subgame-perfect Nash equilibria.

\subsection{Consumers' choice}

Firstly, we observe that if a consumer with identity $x$ chooses to buy from the firm, in equilibrium all consumers with identity less than $x$ choose to buy from the firm. Secondly, if a consumer with identity $x$ chooses to use the P2P network, in equilibrium all consumers with identity larger than $x$ choose to do so. This implies that in equilibrium only a few market structures can arise, where market structures can differ in two dimensions: the degree to which consumers are served and the platforms that are actively used. Regarding the first dimension, the market can be fully served or partially served as is displayed in Figure 2.1 for a multi-platformed market. ${ }^{5}$ Regarding the second dimension we can have a multi-platformed market or a single-platformed market with either only the firm or only the P2P network. For the second stage, we can therefore restrict our attention to the six market structures depicted in Table 2.1. Observe that the situation in the graph on the left of Figure 2.1 is denoted by $[S / N]$ and the one on the right by $[S / \phi / N]$.

\footnotetext{
${ }^{5}$ Notice that the vertical intercept of the utility level from the network is endogenously determined by the resulting network size. The quantities $z, z^{S}$ and $z^{N}$ are used for the analysis in the appendices.
} 

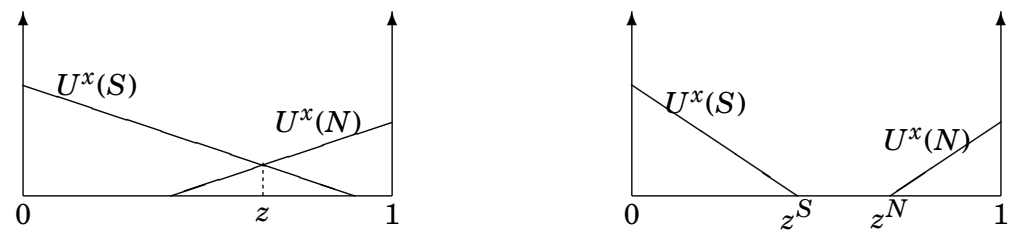

Figure 2.1: Fully-served market and partially-served market.

\begin{tabular}{lccc}
\hline & \multicolumn{2}{c}{ single-platformed } & \\
\cline { 2 - 3 } & firm & network & multi-platformed \\
\hline fully-served & {$[S]$} & {$[N]$} & {$[S / N]$} \\
partially-served & {$[S / \varnothing]$} & {$[\varnothing / N]$} & {$[S / \varnothing / N]$} \\
\hline
\end{tabular}

Table 2.1: All possible market structures in stage two.

Our assumption that $\sigma>\gamma$ implies that the generic cost factor of downloading is sufficiently high such that when the network size is zero, no consumer can join the network. This implies that if everybody expects that nobody will join the network, we have a second-stage equilibrium without network.

Now consider the case where the price set by the firm in stage one is high enough to be consistent with the formation of a P2P network. The size of the smallest network that can be supported as a second-stage equilibrium given some price set by the firm is called the critical-mass network and denoted by c.m. If all the consumers expect that the network is like this, such expectations are self-enforcing. There is one consumer who is indifferent between buying and downloading and whose choice is irrelevant since we have a continuum of consumers. All consumers with higher utility from downloading are strictly better off joining the network and all the other consumers are strictly better off buying from the firm.

Since we have positive downloading externalities, the critical-mass network is destabilized when slightly more consumers, located closest to the indifferent consumer, join it. If all consumers expect that the network is like this, the consumer with the highest value of $x$ buying at the firm will strictly prefer to download, and these expectations are not compatible with 
a second-stage equilibrium. In that case, there will be a maximum network, denoted by $m . n$. that can be supported as a second-stage equilibrium, where the maximal network may be the one with all consumers in it.

The next proposition describes the possible second-stage equilibrium market structures conditional on the first-stage price set by the firm.

Proposition 2.3.1. Given the first-stage price $p$, the possible equilibrium structures that can arise in the second stage are

$[S]$

$$
\text { if } 0 \leq p \leq \beta-\tau \text {, }
$$

$[S / \varnothing]$

if $\beta-\tau<p \leq \beta$,

$[S / N]_{c . m .}$

if $\beta-\gamma+\tau-\frac{\tau^{2}}{\sigma} \leq p \leq \beta-\frac{\tau^{2}}{2 \sigma}\left(1+\sqrt{1+4 \frac{\sigma}{\tau^{2}}(\gamma-\tau)}\right)$,

$[S / \varnothing / N]_{c . m}$.

if $\beta-\frac{\tau^{2}}{2 \sigma}\left(1+\sqrt{1+4 \frac{\sigma}{\tau^{2}}(\gamma-\tau)}\right)<p \leq \beta$,

$[S / N]_{m . n .}$

if $\beta-\gamma+\tau-\frac{\tau^{2}}{\sigma} \leq p \leq \beta-\gamma+\tau$, and

$[N]$

if $\beta-\gamma+\tau<p \leq \beta$.

Proof.

$[\varnothing]$ and $[\varnothing / N]$. Observe that for these structures to occur, the consumer with identity 0 would get a negative utility from buying from the firm, which implies $p>\beta$. This case has been excluded.

[S]. Since $\sigma>\gamma$, this structure is consistent with an equilibrium if and only if the consumer with identity 1 is indifferent between not acquiring the product and buying from the firm, so $\beta-\tau-p \geq 0$, which is the case if and only if $0 \leq p \leq \beta-\tau$.

$[S / \varnothing]$. Since $\sigma>\gamma$, for this structure to occur, the consumer with identity 1 should get a negative utility from buying from the firm, which is the case if and only if $p>\beta-\tau$.

$[S / N]$. See Appendix 2.8.1.

$[S / \varnothing / N]$. See Appendix 2.8.2.

$[N]$. Note that for all Nash equilibria in this structure $n(p, d)=1$, so the costs of downloading are zero. Since $\gamma>\tau$, this structure appears as an equilibrium if and only if the consumer with identity 0 prefers the network over the firm, which is the case if and only if $p>\beta-\gamma+\tau$. 
A closer look at Proposition 2.3.1 reveals that the second-stage equilibrium manifold consists of three segments of logically connected equilibrium structures. They are: (a) the no-network segment: $[S]-[S / \varnothing]$, (b) the criticalmass-network segment: $[S / N]_{c . m .}-[S / \varnothing / N]_{c . m .}$, and (c) the maximum-network segment: $[S / N]_{m . n .}-[N]$. A graphical representation of these segments is provided in Figure 2.2. As we will explain later, the parameters give rise to five possible scenarios, two of which are illustrated in Figure 2.2.

(a) $[S]$

$[S / \varnothing]$

(b) $[S / N]_{\text {c.m. }}$.

(b) $[S / \varnothing / N]_{c . m}$

(c) $[S / N]_{m . n}$.
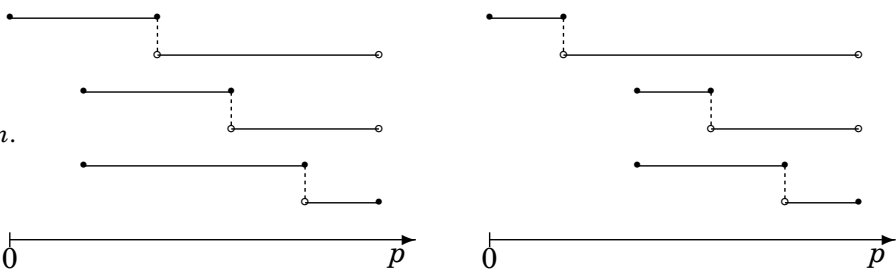

Figure 2.2: scenario A.1 (left); scenario B.1 (right).

The three segments share the common property that they begin at a certain price with one of the equilibrium structures, switch to the other equilibrium structure at a higher price and end with that second structure at an even higher price. Table 2.2 summarizes, for each of the three segments, the starting, switching and ending prices, which are denoted by $p^{\vdash}, p^{\times}$and $p^{-1}$ respectively.

\begin{tabular}{llll}
\hline segment & starting price $\left(p^{\vdash}\right)$ & switching price $\left(p^{\times}\right)$ & ending price $\left(p^{\dashv}\right)$ \\
\hline (a) & 0 & $\beta-\tau$ & $\beta$ \\
(b) & $\beta-\gamma+\tau-\frac{\tau^{2}}{\sigma}$ & $\beta-\frac{\tau^{2}}{2 \sigma}\left(1+\sqrt{1+4 \frac{\sigma}{\tau^{2}}(\gamma-\tau)}\right)$ & $\beta$ \\
(c) & $\beta-\gamma+\tau-\frac{\tau^{2}}{\sigma}$ & $\beta-\gamma+\tau$ & $\beta$ \\
\hline
\end{tabular}

Table 2.2: Three segments of equilibrium structures.

Notice that up to a price of $p_{(b, c)}^{\vdash}=\beta-\gamma+\tau-\tau^{2} / \sigma$ there is a unique equilibrium where all consumers choose to buy from the firm, and that for higher prices there are three equilibria-one for each segment. At the price $p_{(b, c)}^{\vdash}$ there are two equilibria since the one corresponding to the critical-mass net- 
work coincides with the one of the maximum network. It is easily verified that for parameter settings satisfying Assumptions 2.2.1-2.2.3, for each segment, the switching point is strictly between the starting point and the ending point. Moreover, the switching point $p_{(b)}^{\times}$of the critical-mass-network segment exceeds the switching point $p_{(a)}^{\times}$of the no-network segment.

At the no-network segment only one platform is active, the firm. The switching point indicates the price level at which the market switches from being fully served to partially served. At the critical-mass-network segment, the two platforms co-exist. Here, again, the switching point indicates a switch from a fully-served to a partially-served market. For the maximum-network segment, the switching point indicates a switch from a multi-platformed to a single-platformed market. Along this whole segment the market is fully served.

Different parameter values of $\beta, \gamma, \sigma$ and $\tau$ can lead to five scenarios, depending on how the starting point $p_{(b, c)}^{\vdash}$ of both network segments is related to the switching point $p_{(a)}^{\times}$of the no-network segment and how the switching point $p_{(c)}^{\times}$of the maximum-network segment is positioned relative to the switching point of the other two segments. In the three A scenarios, the starting point $p_{(b, c)}^{\vdash}$ of both network segments is less than or equal to the switching point $p_{(a)}^{\times}$of the no-network segment, and the reverse holds for the two B scenarios. The A scenarios result when $\gamma \geq 2 \tau-\tau^{2} / \sigma$ and the B scenarios do when $\gamma<2 \tau-\tau^{2} / \sigma$.

Since the switching point $p_{(b)}^{\times}$of the critical-mass-network segment exceeds the switching point $p_{(a)}^{\times}$of the no-network segment, the switching point $p_{(c)}^{\times}$of the maximum-network segment can be greater than or equal to $p_{(b)}^{\times}$, which is the case if and only if $\gamma \leq 2 \tau$ and $\left(\gamma \leq \tau^{2} / 2 \sigma+\tau\right.$ or $\left.\gamma \geq 2 \tau^{2} / \sigma+\tau\right)$, the switching point $p_{(c)}^{\times}$can belong to the interval $\left[p_{(a)}^{\times}, p_{(b)}^{\times}\right]$, which is the case if and only if $\gamma \leq 2 \tau$ and $\tau^{2} / 2 \sigma+\tau \leq \gamma \leq 2 \tau^{2} / \sigma+\tau$, and the switching point $p_{(c)}^{\times}$can be less than or equal to $p_{(a)}^{\times}$, which is the case if and only if $\gamma \geq 2 \tau$. In this way we obtain three subcases for each of the two scenarios, resulting in scenarios A.1, A.2, A.3, and in scenarios B.1, B.2, and B.3, where the set of parameter values giving rise to B.3 is void. Table 2.3 presents the parameter values that give rise to the five possible scenarios, where we have eliminated redundant constraints. Figure 2.2 illustrates scenarios A.1 and B.1. 


\begin{tabular}{cllll}
\hline scenario & \multicolumn{2}{l}{ location of switching points } & parameter values \\
\hline A.1 & $p_{(b, c)}^{\vdash} \leq p_{(a)}^{\times}$ & $p_{(c)}^{\times} \geq p_{(b)}^{\times}$ & $\gamma \geq 2 \tau-\frac{\tau^{2}}{\sigma}$ & $\gamma \leq 2 \tau \quad \gamma \geq \frac{2 \tau^{2}}{\sigma}+\tau$ \\
A.1 & & & $\gamma \geq 2 \tau-\frac{\tau^{2}}{\sigma}$ & $\gamma \leq \frac{\tau^{2}}{2 \sigma}+\tau$ \\
A.2 & $p_{(b, c)}^{\vdash} \leq p_{(a)}^{\times}$ & $p_{(c)}^{\times} \in\left[p_{(a)}^{\times}, p_{(b)}^{\times}\right]$ & $\gamma \geq 2 \tau-\frac{\tau^{2}}{\sigma}$ & $\frac{\tau^{2}}{2 \sigma}+\tau \leq \gamma \leq \frac{2 \tau^{2}}{\sigma}+\tau$ \\
A.3 & $p_{(b, c)}^{\vdash} \leq p_{(a)}^{\times}$ & $p_{(c)}^{\times} \leq p_{(a)}^{\times}$ & $\gamma \geq 2 \tau$ & \\
B.1 & $p_{(b, c)}^{\vdash}>p_{(a)}^{\times}$ & $p_{(c)}^{\times} \geq p_{(b)}^{\times}$ & $\gamma<2 \tau-\frac{\tau^{2}}{\sigma}$ & $\gamma \geq \frac{2 \tau^{2}}{\sigma}+\tau$ \\
B.1 & & & $\gamma<2 \tau-\frac{\tau^{2}}{\sigma}$ & $\gamma \leq \frac{\tau^{2}}{2 \sigma}+\tau$ \\
B.2 & $p_{(b, c)}^{\vdash}>p_{(a)}^{\times}$ & $p_{(c)}^{\times} \in\left[p_{(a)}^{\times}, p_{(b)}^{\times}\right]$ & $\gamma<2 \tau-\frac{\tau^{2}}{\sigma}$ & $\frac{\tau^{2}}{2 \sigma}+\tau \leq \gamma \leq \frac{2 \tau^{2}}{\sigma}+\tau$ \\
\hline
\end{tabular}

Table 2.3: Possible scenarios and corresponding parameter settings.

Notice that all inequalities in Table 2.3 only involve the parameters $\gamma, \tau$ and $\sigma$, and are independent of $\beta$. Moreover, notice that all inequalities are homogeneous such that we can assume $\sigma=1$ without loss of generality. That is, whenever $(\gamma, \tau, \sigma)$ satisfies the conditions of a particular scenario, so does $\left(\frac{\gamma}{\sigma}, \frac{\tau}{\sigma}, 1\right)$. For $\sigma=1$, Figure 2.3 displays, in $(\tau, \gamma)$-space, how the scenarios are related to one another.

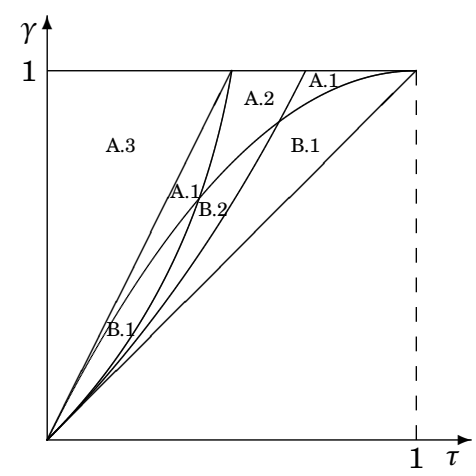

Figure 2.3: Parameter settings leading to each of the scenarios.

In Figure 2.3, the concave-shaped curve separates the A scenarios, with $p_{(b, c)}^{\vdash} \leq p_{(a)}^{\times}$, from the B scenarios, where $p_{(b, c)}^{\vdash}>p_{(a)}^{\times}$. Roughly speaking, $\gamma$ and $\tau$ have to be relatively close for the B scenarios to occur, and conversely $\gamma$ has to be sufficiently larger than $\tau$ for the A scenarios to occur. Intuitively, when $\gamma$ is large compared to $\tau$, the desirability of the digital form dominates 
the costs caused by taste heterogeneity, which makes people located closer to the physical form more prone to downloading. This leads to the A scenarios, where the firm perceives a lot of pressure from the network, i.e. even when the firm sets a low price, inducing a fully-served market, a network may form.

Finally, before moving on to the analysis of stage one, the following proposition specifies (independent of the scenarios) the behavior of the firm's profit along each of the segments. Its proof is straightforward when using the expressions for the consumer that is indifferent between her first-best - buying from the firm - and her second-best choice, as derived in Appendices 2.8.1 and 2.8.2.

\section{Proposition 2.3.2.}

- Along each segment the profit of the firm is continuous in price.

- For each price, the profit on the no-network segment is larger than the profit on the critical-mass-network segment, which is in turn larger than the profit on maximum-network segment.

- At the ending point the profit is zero on each segment.

- Along the no-network segment the profit is equal to the price until the switching point and is concave afterwards.

- The profit at the starting point on both network segments is equal to $\frac{\tau}{\sigma}$ times the price.

- Along the maximum-network segment the profit is zero after the switching point.

Intuitively, for a given price with multiple equilibria in the second stage, the firm always has a higher profit if the network is not formed. At the ending point of each segment the price is $\beta$, which will result in no sales, and hence zero profit. Before the switching point on the no-network segment, the sales quantity is exactly one (the full market), therefore the profit is equal to the price. After the switching point, the sales quantity decreases in price and the profit is therefore concave in price. At the starting point 
of both network segments, the network size is identical for the critical-mass network and the maximum network, and the sales quantity is less than one. After this starting point, the size of the critical-mass network starts to decrease and the size of the maximum network starts to increase. The profit along the maximum-network segment after the switching point is zero because there are no sales. The profits along the no-network segment (a), the critical-mass-network segment (b), and the maximum-network segment (c) are illustrated in Figure 2.4. Note that in Figure 2.4, the profit on the nonetwork segment (a) at a price equal to $p_{(b, c)}^{\vdash}$ is not guaranteed to exceed the global maximum of the profit on the maximum-network segment (c). Moreover, in the B scenarios it is possible that the global maximum of the profit on the no-network segment (a) is attained at a price below $p_{(b, c)}^{\vdash}$.
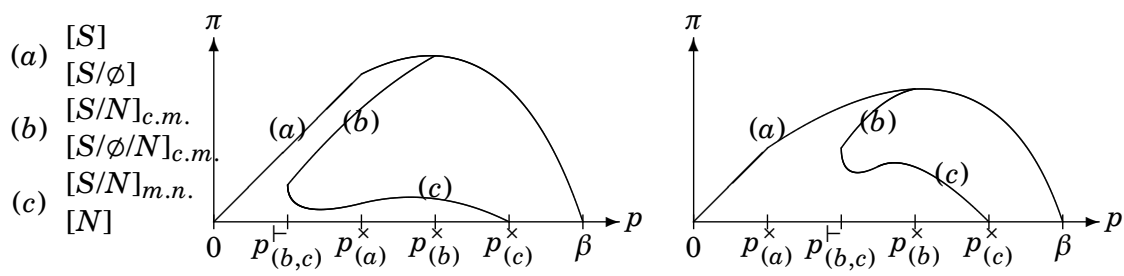

Figure 2.4: Profit along segments; scenario A.1 (left); scenario B.1 (right).

\subsection{The firm's decision}

\subsubsection{Subgame-perfect Nash equilibria}

Using the definition of a subgame-perfect Nash equilibrium and the results of the previous section, the following result is immediate.

Proposition 2.4.1. The strategy profile $\left(p^{*}, d^{*}\right)$ is a subgame-perfect Nash equilibrium if and only if, for every $p \in[0, \beta], d^{*}(p)$ is consistent with one of the second-stage equilibrium structures of Proposition 2.3.1 and $\pi\left(p^{*}, d^{*}\left(p^{*}\right)\right)$ $\geq \pi\left(p, d^{*}(p)\right)$.

In order to analyze which pairs of prices and consumer decisions $(p, d(p))$ can be supported as a subgame-perfect equilibrium outcome, it is convenient to define the firm-worst response of the consumers. Let therefore 
$d_{\mathrm{LE}}$ be the mapping that assigns to each price the equilibrium structure that generates the lowest profit for the firm, except at the starting price $p_{(b, c)}^{\vdash}=\beta-\gamma+\tau-\tau^{2} / \sigma$ of the network segments, where for later convenience we define $d_{\mathrm{LE}}(p)$ as the no-network equilibrium structure, so $d_{\mathrm{LE}}^{x}(p)=S$, $x \in[0,1]$. LE stands for "lower envelope". For the five scenarios depicted in Table 2.3, the corresponding lower-envelope mappings are depicted in Figure 2.5. Notice that the lower envelope of the three A scenarios has
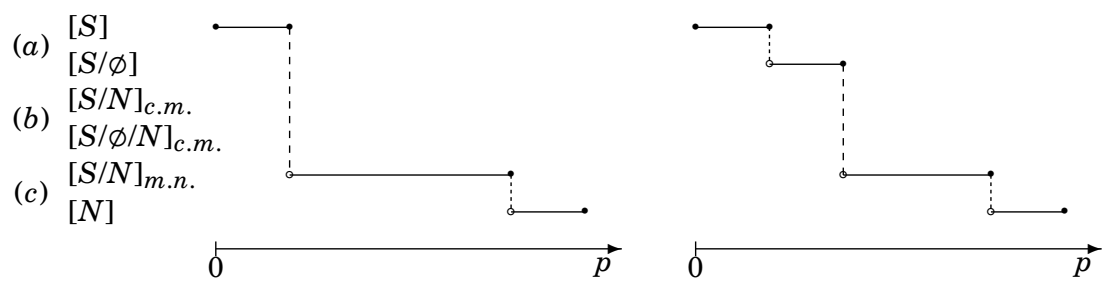

Figure 2.5: The lower envelope of the different scenarios (left: A scenarios; right: B scenarios).

the structure as displayed in the left panel and the two B scenarios as displayed in the right panel. Moreover, the lower envelope only involves the no-network segment and the maximum-network segment, but not the critical-mass-network segment. We define the maximum profit along the lower envelope by $\pi_{\mathrm{LE}}^{*}=\max _{p} \pi\left(p, d_{\mathrm{LE}}(p)\right)$. Proposition 2.3 .2 and the definition of the lower envelope guarantees that the maximum exists, even though $\pi\left(p, d_{\mathrm{LE}}(p)\right)$ is not everywhere continuous in $p$. We have the following characterization of subgame-perfect equilibrium outcomes.

Proposition 2.4.2. The pair $\left(p^{*}, d^{*}\left(p^{*}\right)\right)$ is a subgame-perfect Nash equilibrium outcome if and only if $d^{*}\left(p^{*}\right)$ is consistent with one of the second-stage equilibrium structures of Proposition 2.3.1 and $\pi\left(p^{*}, d^{*}\left(p^{*}\right)\right) \geq \pi_{\mathrm{LE}}^{*}$

Proof.

$(\Rightarrow)$

Let $\left(p^{*}, d^{*}\right)$ be a subgame-perfect Nash equilibrium with equilibrium outcome $\left(p^{*}, d^{*}\left(p^{*}\right)\right)$. Proposition 2.4.1 implies that $d^{*}\left(p^{*}\right)$ is consistent with one of the second-stage equilibrium structures of Proposition 2.3.1. Since, given $d^{*}$ the firm has no incentive to deviate from $p^{*}$, we have, for every 
$p \in[0, \beta]$,

$$
\pi\left(p^{*}, d^{*}\left(p^{*}\right)\right) \geq \pi\left(p, d^{*}(p)\right) .
$$

For $p \in[0, \beta] \backslash\left\{p_{(b, c)}^{\vdash}\right\}$, it holds that $\pi\left(p, d^{*}(p)\right) \geq \pi\left(p, d_{\mathrm{LE}}(p)\right)$, so

$$
\begin{aligned}
\pi\left(p^{*}, d\left(p^{*}\right)\right) & \geq \sup _{p \in[0, \beta] \backslash\left\{p_{(b, c)}^{\vdash}\right\}} \pi\left(p, d_{\mathrm{LE}}(p)\right) \\
& =\sup _{p \in[0, \beta]} \pi\left(p, d_{\mathrm{LE}}(p)\right) \\
& =\pi_{\mathrm{LE}}^{*},
\end{aligned}
$$

where the first inequality uses that $\pi\left(p, d_{\mathrm{LE}}(p)\right)$ is continuous from the left at $p_{(b, c)}^{\vdash}$.

$(\Leftarrow)$

We define the consumers' strategy profile $\widetilde{d}_{\mathrm{LE}}$ by

$$
\widetilde{d}_{\mathrm{LE}}\left(p^{*}\right)=d^{*}\left(p^{*}\right) \text { and } \widetilde{d}_{\mathrm{LE}}(p)=d_{\mathrm{LE}}(p) \text { for } p \neq p^{*}
$$

By Proposition 2.4.1, $\left(p^{*}, \widetilde{d}_{\mathrm{LE}}\right)$ is a subgame-perfect Nash equilibrium, and therefore $\left(p^{*}, d^{*}\left(p^{*}\right)\right)$ a subgame-perfect Nash equilibrium outcome, if

$$
\pi\left(p^{*}, \widetilde{d}_{\mathrm{LE}}\left(p^{*}\right)\right) \geq \pi\left(p, \widetilde{d}_{\mathrm{LE}}(p)\right) \quad \text { for every } \quad p \in[0, \beta] .
$$

This inequality holds since, by assumption, $\pi\left(p^{*}, \widetilde{d}_{\mathrm{LE}}\left(p^{*}\right)\right) \geq \pi_{\mathrm{LE}}^{*}$, and $\pi_{\mathrm{LE}}^{*} \geq$ $\pi\left(p, \widetilde{d}_{\mathrm{LE}}(p)\right)$ for $p \neq p^{*}$ by definition of $\pi_{\mathrm{LE}}^{*}$.

\subsubsection{Equilibrium selection}

To deal with the multiplicity of subgame-perfect equilibria, we follow the convention by supposing that, once the price is known, consumers coordinate on the equilibrium continuation that they prefer, which is the one with the largest network size. ${ }^{6}$ To avoid continuity problems, we assume coordination on the firm for the knife-edge case when the price equals $p_{(b, c)}^{\vdash}$. This corresponds to the firm-worst response of the consumers, which is captured by our lower envelope. In this subsection, we therefore study the firm's optimal pricing behavior along the lower envelope. Guided by Figure 2.5, we treat the A scenarios and the B scenarios separately, and refer to them as scenario A and scenario B, respectively.

\footnotetext{
${ }^{6}$ See, for instance, (Katz and Shapiro 1986) or (Fudenberg and Tirole 2000).
} 
For the sake of convenience in notation, we define $\delta \equiv 4 \tau^{2}-3 \sigma(\beta-\gamma+\tau)$. Roughly speaking, $\delta$ represents the attractiveness of the network relative to the firm, as it is increasing in $\gamma-\beta$ and decreasing in $\sigma$. Without loss of generality, we assume that when different first-stage prices lead to identical second-stage profit levels, the firm selects the price that results in the smallest network.

Scenario A. In scenario A, the firm's profit along the lower envelope is given by:

$$
\pi\left(p, d_{\mathrm{LE}}(p)\right)= \begin{cases}p & \text { if } 0 \leq p \leq p_{(b, c)}^{\vdash} \\ p \cdot \frac{\tau-\sqrt{\sigma(p+\gamma-\beta-\tau)+\tau^{2}}}{\sigma} & \text { if } p_{(b, c)}^{\vdash}<p \leq p_{(c)}^{\times} \\ 0 & \text { if } p_{(c)}^{\times}<p \leq \beta .\end{cases}
$$

The profit in the interval $[N]$ is zero. The profit in the interval $[S]$ reaches its maximum at the right boundary point $p_{(b, c)}^{\vdash}$, which we denote by $\pi_{[S]}^{*}=$ $\beta-\gamma+\tau-\tau^{2} / \sigma$. The profit in the interval $[S / N]_{m . n}$. starts at $\frac{\tau}{\sigma}\left(\beta-\gamma+\tau-\tau^{2} / \sigma\right)$, which is a share $\frac{\tau}{\sigma}$ of $\pi_{[S]}^{*}$. At this point it decreases with a slope of $-\infty$, and ends up equal to zero at the price $p_{(c)}^{\times}=\beta-\gamma+\tau$. However, before it reaches zero, it may increase and then subsequently decrease, achieving a local maximum at the price

$$
p_{[S / N]_{m . n .}}^{*}=\frac{2}{9 \sigma}(2 \tau-\sqrt{\delta})(\tau+\sqrt{\delta}) .
$$

This expression is only valid if $\delta$ is non-negative, which incidentally also guarantees that the price is in the respective interval, i.e. $\delta \geq 0$ implies $p_{(b, c)}^{\vdash}<p_{[S / N]_{m . n .}}^{*}<p_{(c)}^{\times}$. In that case, the profit at this local maximum is

$$
\pi_{[S / N]_{m . n .}}^{*}=\frac{2}{27 \sigma^{2}}(2 \tau-\sqrt{\delta})^{2}(\tau+\sqrt{\delta}) .
$$

Thus, the firm sets a price of $p_{[S / N]_{m . n .}}^{*}$ if and only if $\delta \geq 0$ and $\pi_{[S / N]_{m . n}}^{*}>\pi_{[S]}^{*}$. This leads to a multi-platformed fully-served market with co-existence of the firm and the network. If $\delta<0$, or in case $\delta \geq 0$ but $\pi_{[S / N]_{m . n .}}^{*} \leq \pi_{[S]}^{*}$, the firm sets a network deterring price equal to $p_{(b, c)}^{\vdash}$, which leads to a singleplatformed fully-served market with network-deterring pricing. In this case the firm selects the network-deterring price rather than the co-existence price when both prices lead to the same profit level, due to the assumption mentioned above. 
Scenario B. In scenario B, the firm's profit along the lower envelope is given by:

$$
\pi\left(p, d_{\mathrm{LE}}(p)\right)= \begin{cases}p & \text { if } 0 \leq p \leq p_{(a)}^{\times} \\ p \cdot \frac{\beta-p}{\tau} & \text { if } p \times(a)<p \leq p_{(b, c)}^{\vdash} \\ p \cdot \frac{\tau-\sqrt{\sigma(p+\gamma-\beta-\tau)+\tau^{2}}}{\sigma} & \text { if } p_{(b, c)}^{\vdash}<p \leq p_{(c)}^{\times} \\ 0 & \text { if } p_{(c)}^{\times}<p \leq \beta\end{cases}
$$

Again, the profit in the interval $[N]$ is zero. In the interval $[S]$, the maximum profit is achieved at a price equal to $p_{(a)}^{\times}$. This is a global maximum on the whole lower envelope if and only if $\beta \geq 2 \tau$. In this case, the firm optimally serves the whole market. Next, in the interval $[S / \varnothing]$, the maximum profit is achieved at the price $p=\beta / 2$. This is the global maximum on the lower envelope if and only if $2\left(\gamma-\tau+\tau^{2} / \sigma\right)<\beta<2 \tau$. In that case, the firm optimally serves the market partially as a monopoly, and there will be no network.

If $\beta \leq 2\left(\gamma-\tau+\tau^{2} / \sigma\right)$, the situation is similar to scenario $\mathrm{A}$. The maximum profit in the interval $[S / \varnothing]$ is achieved at the price $p_{(b, c)}^{\vdash}=\beta-\gamma+\tau-\tau^{2} / \sigma$, yielding $\pi_{[S / \varnothing]}^{*}=\left(\beta-\gamma+\tau-\tau^{2} / \sigma\right)(\gamma / \tau-1+\tau / \sigma)$. Thus, the firm sets the price at $p=2(2 \tau-\sqrt{\delta})(\tau+\sqrt{\delta}) / 9 \sigma$ if and only if $\delta \geq 0$ (which guarantees $p_{(b, c)}^{\vdash}<$ $\left.p_{[S / N]_{m . n .}}^{*}<p_{(c)}^{\times}\right)$and $\pi_{[S / N]_{m . n .}}^{*}>\pi_{[S / \phi]}^{*}$. This leads to a multi-platformed fullyserved market with the co-existence of the firm and the network. Otherwise the firm sets a network-deterring price equal to $p_{(b, c)}^{\vdash}$, which leads to a single-platformed partially-served market with network-deterring pricing.

Observe that, in scenario $\mathrm{B}$, on top of the network-deterring pricing and co-existence cases like in scenario A, we have two cases of monopoly pricing, under the condition that $\beta$ is sufficiently high when compared to $\tau$. Notice also that the network-deterring pricing in scenario B occurs in a partiallyserved market as opposed to a fully-served market in scenario A.

We summarize the findings from our studies of the scenarios above in the following propositions.

Proposition 2.4.3. The firm acts as a monopolist if and only if

$$
\gamma<2 \tau-\frac{\tau^{2}}{\sigma} \text { and } \beta>2\left(\gamma-\tau+\frac{\tau^{2}}{\sigma}\right) .
$$

If in addition $\beta \geq 2 \tau$, the market is fully served; otherwise it is partially served. 
The first inequality in the proposition ensures that we are in scenario B.

The proposition indicates that when the quality of the physical form of the product is sufficiently high and the one of the digital form is sufficiently low, the firm can monopolize the market. In case the gross value of the product exceeds by far the transportation costs caused by taste heterogeneity, the firm is willing to serve the whole market. When this is not the case, the firm does not attempt to sell to the consumers with strong preferences for the digital form. Notice that, here, in its pricing the firm disregards the network. This is not the case in the next proposition.

Proposition 2.4.4. There are parameter constellations such that, in equilibrium, the firm applies network-deterring pricing. This is the case with a fully-served market if and only if

$$
\gamma \geq 2 \tau-\frac{\tau^{2}}{\sigma} \quad \text { and } \quad\left(\delta<0 \text { or } \pi_{[S / N]_{m . n .}}^{*} \leq \pi_{[S]}^{*}\right),
$$

and the case with a partially-served market if and only if

$$
\gamma<2 \tau-\frac{\tau^{2}}{\sigma}, \quad \beta \leq 2\left(\gamma-\tau+\frac{\tau^{2}}{\sigma}\right), \quad \text { and } \quad\left(\delta<0 \text { or } \pi_{[S / N]_{m . n .}}^{*} \leq \pi_{[S / \phi]}^{*}\right) .
$$

When the digital form is above a certain quality level, or when the physical form is below a certain quality level, the network has sufficient potential to form and the firm is no longer able to apply monopoly pricing. Stated differently, the firm is disciplined by the threat of the network forming. However, when the generic cost factor of downloading $\sigma$ is sufficiently large (such that $\delta$ is negative), the firm is able to price as such to deter the network from developing.

Proposition 2.4.5. The firm and the network co-exist if and only if the conditions in the previous two propositions are not met. This is the case if and only if

$$
\gamma \geq 2 \tau-\frac{\tau^{2}}{\sigma}, \quad \delta \geq 0, \quad \text { and } \quad \pi_{[S / N]_{m . n .}}^{*}>\pi_{[S]}^{*}
$$

or

$$
\gamma<2 \tau-\frac{\tau^{2}}{\sigma}, \quad \delta \geq 0, \quad \text { and } \quad \pi_{[S / N]_{m . n .}}^{*}>\pi_{[S / \phi]}^{*} .
$$

In such a case, the market is fully served. 
Proof. It holds that the conditions in Proposition 2.4.3 and 2.4.4 are not met if and only if

$$
\gamma \geq 2 \tau-\frac{\tau^{2}}{\sigma}, \quad \delta \geq 0, \quad \text { and } \quad \pi_{[S / N]_{m . n .}}^{*}>\pi_{[S]}^{*}
$$

or

$$
\gamma<2 \tau-\frac{\tau^{2}}{\sigma}, \quad \beta \leq 2\left(\gamma-\tau+\frac{\tau^{2}}{\sigma}\right), \quad \delta \geq 0, \quad \text { and } \quad \pi_{[S / N]_{m . n .}}^{*}>\pi_{[S / \varnothing]}^{*} .
$$

Now $\delta \geq 0$ implies

$$
\beta \leq \frac{4 \tau^{2}}{3 \sigma}+\gamma-\tau<\frac{2 \tau^{2}}{\sigma}+2(\gamma-\tau),
$$

so we can omit the condition on $\beta$.

The three propositions above indicate that the parameter $\delta=4 \tau^{2}-3 \sigma(\beta-$ $\gamma+\tau$ ) plays an important role in determining market outcomes. Recall that $\delta$ corresponds to the attractiveness of the network relative to the firm. For the conditions in Proposition 2.4.5 to be satisfied, $\delta$ must be positive. Thus, for the two platforms to co-exist, the two forms of the product should not be too distinct in quality and the generic cost factor of downloading should be sufficiently low. It can be shown that the inequalities in Proposition 2.4.3, where monopoly is the equilibrium market outcome, require $\delta$ to be negative.

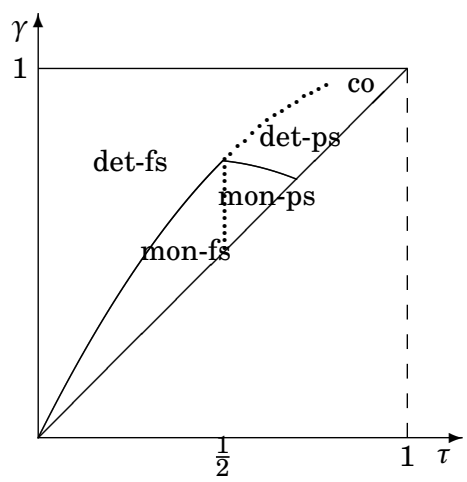

Figure 2.6: A graphical illustration of the possible subgame-perfect Nash equilibrium market structures.

We close this section with a graphical illustration of how the different equilibrium market structures are located in the parameter space. For the case with both $\beta$ and $\sigma$ equal to 1 , Figure 2.6 presents the areas described 
in the propositions in ( $\tau, \gamma)$-space, where 'mon', 'det' and 'co' refer to monopoly (Proposition 2.4.3), network-deterring pricing (Proposition 2.4.4) and co-existence (Proposition 2.4.5) respectively, and ' $\mathrm{fs}$ ' and 'ps' refer to a fully-served and partially-served market respectively.

The concave curve is identical to the one in Figure 2.3, and separates scenario A (to the left of the curve) from scenario B (to the right of the curve). A monopoly can only exist in scenario B, either if $\tau \leq \frac{1}{2}$ (fully-served market), or if $\tau>\frac{1}{2}$ and $2\left(\gamma-\tau+\tau^{2}\right)<1$ (partially-served market). This suggests that for the monopoly to exist, $\gamma$ should not be too high when compared to $\beta$, so the quality of the physical form of the product should be sufficiently superior to the one of the digital form. Furthermore, the ratio $\frac{\gamma}{\tau}$ should not be too high, which means that sufficient taste heterogeneity among the consumers is necessary. Co-existence, on the other hand, occurs both in scenario A and $\mathrm{B}$, but only in the far upper-right corner. This requires $\tau$ to be sufficiently large, which intuitively suggests that when taste heterogeneity is large, it does not pay off for the firm to deter the network from developing. Hence, the firm will accommodate the existence of the network. Everywhere else we find monopoly with network-deterring pricing in either a fully-served (in scenario A) or partially-served market (in scenario B). This shows that there is quite some room for the firm to manipulate the price and deter the development of the network.

\subsection{Comparative statics}

In this section, we conduct some comparative-static analysis on the equilibrium outcomes of the subgame-perfect Nash equilibria discussed in the previous section. We will treat them separately according to their market structures. Recall that $p^{*}, s^{*}, n^{*}$, and $\pi^{*}$ are the equilibrium price, the sales quantity of the firm, the network size, and the firm's profit respectively. $C S^{*}$ and $W^{*}$ denote the equilibrium consumers' surplus and the total welfare respectively. 


\subsubsection{Monopoly}

Proposition 2.5.1. The signs of the first-order partial derivatives of the equilibrium values with respect to the parameters in the case of the monopoly market are as shown in Table 2.4. ${ }^{7}$

\begin{tabular}{lcccc}
\hline & $\beta$ & $\gamma$ & $\tau$ & $\sigma$ \\
\hline$p^{*}$ & + & 0 & - & 0 \\
$s^{*}$ & 0 & 0 & 0 & 0 \\
$n^{*}$ & 0 & 0 & 0 & 0 \\
$\pi^{*}$ & + & 0 & - & 0 \\
$C S^{*}$ & 0 & 0 & + & 0 \\
$W^{*}$ & + & 0 & - & 0 \\
\hline
\end{tabular}

\begin{tabular}{lcccc}
\hline & $\beta$ & $\gamma$ & $\tau$ & $\sigma$ \\
\hline$p^{*}$ & + & 0 & 0 & 0 \\
$s^{*}$ & + & 0 & - & 0 \\
$n^{*}$ & 0 & 0 & 0 & 0 \\
$\pi^{*}$ & + & 0 & - & 0 \\
$C S^{*}$ & + & 0 & - & 0 \\
$W^{*}$ & + & 0 & - & 0 \\
\hline
\end{tabular}

Table 2.4: Monopoly pricing with fully-served market (left); Monopoly pricing with partially-served market (right). The cells display the signs of the first derivatives.

The findings here are intuitive. For example, in a fully-served monopolistic market, the consumers' surplus does not increase when the basic utility of the physical form of the product $\beta$ increases, because the price increases by the same amount. The firm's profit increases and hence total welfare increases. An increase in taste heterogeneity $\tau$ decreases the price by exactly the same amount since the firm must keep the last consumer (whose transportation cost is exactly $\tau$ ) on board. This results in a lower profit for the firm, while consumers benefit from the lower price. In fact, for all but the last consumer the increase in taste heterogeneity is less than the decrease in price. Therefore, the total consumers' surplus increases in taste heterogeneity. Total welfare, however, decreases due to the larger loss in profit. The basic utility of the digital form $\gamma$ and the generic cost factor of downloading $\sigma$ do not influence any of the endogenous variables, since small variations in it do not lead to the establishment of the network.

In the partially-served monopolistic market, a larger basic utility $\beta$ leads to more sales, higher price, higher profit, and higher welfare; whereas a larger taste heterogeneity $\tau$ leads to lower sales, lower profit, and lower

\footnotetext{
${ }^{7}$ The proofs of the signs in the tables in Propositions 2.5.1, 2.5.2 and 2.5.3 are provided in Appendix 2.8.3.
} 
welfare. Unlike in the fully-served monopoly market, the basic utility of the digital form $\gamma$ and the generic cost factor of downloading $\sigma$ do not influence any of the endogenous variables.

\subsubsection{Network-deterring pricing}

Proposition 2.5.2. The comparative statics on the network-deterring pricing market structure are as shown in Table 2.5.

\begin{tabular}{lcccc}
\hline & $\beta$ & $\gamma$ & $\tau$ & $\sigma$ \\
\hline$p^{*}$ & + & - & $+/-$ & + \\
$s^{*}$ & 0 & 0 & 0 & 0 \\
$n^{*}$ & 0 & 0 & 0 & 0 \\
$\pi^{*}$ & + & - & $+/-$ & + \\
$C S^{*}$ & 0 & + & $+/-$ & - \\
$W^{*}$ & + & 0 & - & 0 \\
\hline
\end{tabular}

\begin{tabular}{lcccc}
\hline & $\beta$ & $\gamma$ & $\tau$ & $\sigma$ \\
\hline$p^{*}$ & + & - & $+/-$ & + \\
$s^{*}$ & 0 & + & - & - \\
$n^{*}$ & 0 & 0 & 0 & 0 \\
$\pi^{*}$ & + & - & - & + \\
$C S^{*}$ & 0 & + & $+/-$ & - \\
$W^{*}$ & + & + & - & - \\
\hline
\end{tabular}

Table 2.5: Fully-served market (left); Partially-served market (right). The cells display the signs of the first derivatives.

In case the market is fully served by the firm, the price is the same as the profit. They both increase in the quality of the physical form $\beta$ and decrease in that of the 'rival' digital form $\gamma$, because a lower price is needed to deter the network from forming. Moreover, for the same reason, they both increase in the generic cost factor of downloading $\sigma$. As a result, consumers' surplus increases in $\gamma$, thanks to the lower price charged for the product, but decreases in $\sigma$, due to the higher price. Total welfare increases in $\beta$, thanks to the higher profit, but does not depend on $\gamma$ or $\sigma$. The reason is that in the fully-served market with network-deterring pricing, $\gamma$ and $\sigma$ only have an effect via the price of the product, and therefore only affect the distribution of surplus between the consumers and the firm. An increase in the taste heterogeneity $\tau$ makes consumers less likely to switch platforms, and may lead to either an increase or a decrease in price. When $\sigma$ is relatively low, in particular if $\sigma<2 \tau$, an increase in $\tau$ leads to a lower price, and consequently a lower profit. However, for larger values of $\sigma$, the price and the profit increase when $\tau$ increases. The intuition is that when the taste 
heterogeneity increases, the digital form becomes less attractive for the consumers, an effect which is further amplified via a smaller expected network size. For larger values of the cost factor, high heterogeneity increases the expected costs of downloading sufficiently in order to shade the competition that the firm is facing from the network and allows the firm to increase the price without losing any sales. Although the effect on the consumers' surplus can be positive or negative, total welfare suffers from higher $\tau$.

A difference we notice when the market is only partially served is that the effects of $\gamma$ and $\sigma$ on the the firm' sales quantity change. A decrease in $\gamma$ and an increase in $\sigma$ makes the network less competitive, and triggers an increase in price $p^{*}$, and leads to less sales $s^{*}$. When it comes to the profit, $\pi^{*}$, the price effect dominates the quantity effect. Thus, the comparative statics of prices and profits go in the same direction, similar to the case where the market is fully served. The total welfare $W^{*}$ depends on $\gamma$ and $\sigma$ as well. It goes the opposite direction as the firm's price $p^{*}$. With a lower $\gamma$ or a higher $\sigma$, leading to a higher price, the gain in profit in the partiallyserved market is less than that in the fully-served market, due to a lower sales quantity. The effect on the consumers' surplus being the same, total welfare decreases, which explains the positive sign for $\gamma$ and the negative one for $\sigma$. An increase in $\tau$ also affects sales negatively, but the consequences for the price are again ambiguous. As with the fully-served market, the price decreases if $\sigma<2 \tau$ and increases if $\sigma>2 \tau .^{8}$ An increase in $\tau$ may now lead to either higher or lower consumers' surplus, depending on the quantitative significance of the decrease in sales, the increase in transportation cost, and the change in price. The change in total welfare is unambiguously negative when $\tau$ increases.

Even when the network does not form, its attractiveness (represented by a high $\gamma$ and a low $\sigma$ ) does have a negative impact on the firm's profit and a positive impact on the total welfare. This has some implications for intellectual property rights policy, which we will discuss later.

\footnotetext{
${ }^{8}$ Both inequalities on parameter values are consistent with the conditions of Proposition 2.4.4.
} 


\subsubsection{Co-existence}

Proposition 2.5.3. The comparative statics of the co-existence of the firm and the network in a fully-served market are as shown in Table 2.6.

\begin{tabular}{lcccc}
\hline & $\beta$ & $\gamma$ & $\tau$ & $\sigma$ \\
\hline$p^{*}$ & + & - & + & - \\
$s^{*}$ & + & - & - & + \\
$n^{*}$ & - & + & + & - \\
$\pi^{*}$ & + & - & + & + \\
$C S^{*}$ & - & + & - & - \\
$W^{*}$ & + & + & - & - \\
\hline
\end{tabular}

Table 2.6: Co-existence of the firm and the network with a fully-served market. The cells display the signs of the first derivatives.

One can argue that nowadays the co-existence case prevails in reality, probably most prominently so in the music industry. Here we find some very interesting results. An increase in $\beta$ leads to a higher price, sales, profit, and a lower network size. Surprisingly, consumer surplus is affected negatively by an increase in $\beta$. The negative effects of a higher price and a lower network size outweigh the increase in quality $\beta$. Total welfare varies positively with $\beta$.

The comparative statics with respect to $\tau$ confirm the usual intuitions. An increase in $\tau$ leads to a higher price and profit, lower sales, and a bigger network. The consumers' surplus and total welfare are affected negatively.

The equilibrium price $p^{*}$ decreases in the generic cost factor of downloading $\sigma$, despite the fact that the equilibrium network size $n^{*}$ decreases in $\sigma$. This implies that, in equilibrium, the higher the cost factor $\sigma$, the lower the firm sets its price: a counter-intuitive result, since a higher $\sigma$ makes the network less strong as a competitor of the firm. The negative correlation is caused by the shape of the cost function of downloading $C(n)$, which is convex and decreasing in the network size $n$. In other words, since downloading costs decrease with the network size at a diminishing rate, it makes the first few consumers who join the network more vital in determining the costs (or the price, effectively) of downloading. The firm, therefore, has an incentive 
to "play tough" by means of a low price when the equilibrium network size is relatively small (large $\sigma$ ). Conversely, if $\sigma$ is small, the equilibrium network size is large, and the network is stronger and will form more easily. In that case, the firm can do better by backing off from the competition and charging a high price to reap the most profit out of the customers that are more eager to buy the physical form of the product. This can be seen by the equilibrium profit $\pi^{*}$ being increasing in $\sigma$, despite the decreasing price. What we observe here, therefore, is a very interesting form of platform competition. Moreover, similar to the partially-served market network-deterring pricing case, total welfare $W^{*}$ decreases in $\sigma$. This implies that it is welfare enhancing if the internet infrastructure of the country is improved, and legal enforcements of intellectual property rights are relaxed.

\subsubsection{Comparative statics across market structures}

In the above subsections we have shown comparative statics in the three different market structures separately. In this subsection we analyze how equilibrium values change across the market structures as a function of the most important parameter in the model, $\sigma$. We show graphically how equilibrium price, profit and total welfare change in relation to $\sigma$. In order to make a clear sketch, we display the limit case where $\beta, \gamma$ and $\tau$ are equal to 1 . We allow $\sigma$ to vary on the horizontal axis and put the equilibrium values on the vertical axis. Figure 2.7 shows the equilibrium price, profit and welfare responding to changes in $\sigma$.
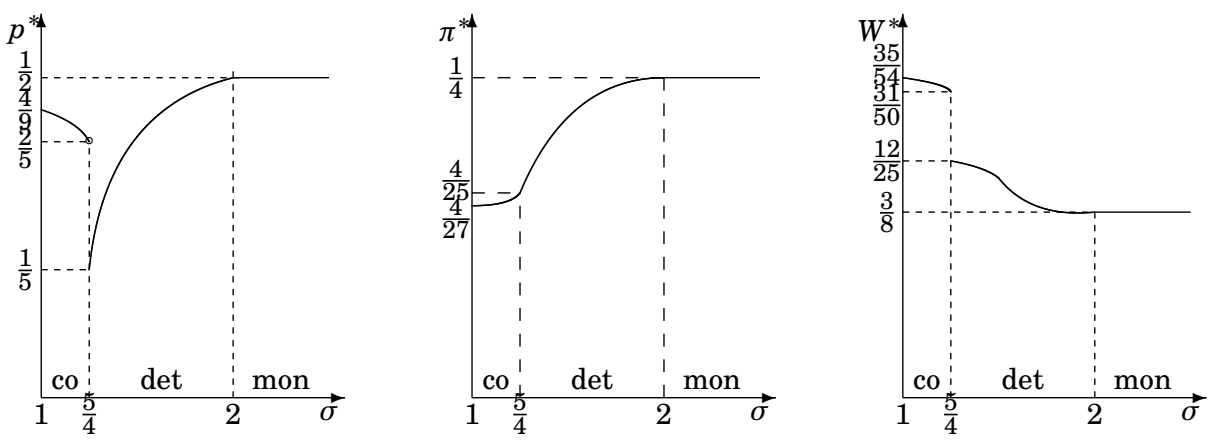

Figure 2.7: Equilibrium price (left), profit (middle), and welfare (right) across markets structures. 
When the generic cost factor of downloading $\sigma$ is very high, the traditional monopoly setting results, as one would expect. As this cost factor decreases, the price, the profit and total welfare remain unaffected as long as the market structure remains unchanged. Once $\sigma$ becomes sufficiently small (less than 2), the firm has to adapt its pricing strategy, because the network becomes a vital enemy to the firm. As long as $\sigma$ is not too small (larger than $\frac{5}{4}$ ), the firm optimally deters the forming of the network. In this region, the price and the profit are decreasing for decreasing values of $\sigma$. The profit is decreasing at an increasing rate. Total welfare, however, increases. In case the cost factor becomes very small $\left(\sigma<\frac{5}{4}\right)$, the firm optimally tolerates the network and refrains from further deterring behavior. This results in a discontinuous increase in price and welfare as the market tips from a single-platformed structure towards a multi-platformed one. From here, the equilibrium price and welfare gradually increase as $\sigma$ decreases. The profit continues to decrease, but only decreases at a diminishing rate.

The comparative statics with a decreasing $\sigma$ can be thought of as what happened in the last decade with the arrival of the P2P networks. We conclude that, although the society as a whole unambiguously benefits from a decrease in the generic cost factor of downloading, the firm unambiguously suffers from it despite a non-monotonic optimal pricing policy. One can, therefore, only expect every attempt possible from the firm to make file-sharing and downloading very difficult and costly indeed.

\subsection{Discussion}

The results of this chapter have a bearing on intellectual property rights policy. We have shown that the total welfare unambiguously increases when the generic cost factor of downloading decreases, in particular, when there is less protection of intellectual property. To give further underpinnings for this conclusion, one would like to extend the model with a part that involves the creation of new content, and analyze how quality and quantity of new content is affected by intellectual property rights. As shown in (Johnson 1985) and (Bae and Choi 2006), these effects are complicated and can depend on various factors, including the nature of piracy costs and the value consumers place on product variety. 
The model and its results presented in this chapter can also be applied outside the context of P2P file-sharing or piracy. In fact, with some modifications, it can be applied to any situation where a monopoly is faced with a free outside option that exhibits positive network externalities. An example would be the competition between commercial software against freely distributed open-source software, for instance the market for operating systems, with Microsoft representing the firm and Linux representing the digital form. The externalities would in this case be related to the amount of support users of a particular platform can expect, as well as the availability of software that is compatible with the operating system chosen. Similar to the case studied in this chapter, the marginal contribution of an additional user to the other users of the platform is diminishing in the size of the network. However, in this application, both platforms would create externalities for its users, which calls for an interesting extension of our model.

Another interesting application would involve an extension of the model of trading platforms as analyzed in (Nocke, Peitz, and Stahl 2007). The authors study the effects of different platform ownership structures on the platform size, as well as on consumer and total surplus. In their model, buyers can either visit the trading platform or take the outside option. Regarding the outside option it is assumed that no externalities are present. Allowing for positive externalities among buyers taking the outside option, would affect the outcome of the trading platform, and it is natural to expect that these externalities interact with the platform ownership structure.

\subsection{Conclusion}

This chapter investigates the competition between a monopolistic information content provider offering the physical form of the product and a P2P file-sharing network (offering the digital form), where consumers that opt for the P2P network benefit from the presence of other consumers due to decreased search costs and downloading time and increased availability. The model has four parameters that capture the quality of the physical form of the product, the quality of the digital form, the level of consumers' taste heterogeneity, and the generic cost factor of downloading. This latter factor incorporates elements such as population computer literacy, the availability 
of broadband internet infrastructure, and in particular, the degree of legal enforcement of intellectual property rights.

We give a complete characterization of the subgame-perfect Nash equilibria of the model as well as of the subgame-perfect Nash equilibrium outcomes. We show that, depending on the parameter values, several market structures may result. These market structures differ not only in the number of platforms being active (one or two) and the level at which the market is served (fully or partially), but also in the firm's pricing policy. A market structure without any network is compatible both with monopoly pricing by the firm as well as with a network-deterring pricing policy. Given fixed values for the other parameters, as we gradually decrease the generic cost factor of downloading, the market experiences first the single platform (the firm) with monopoly pricing, then the single platform (the firm) with network-deterring pricing, and finally co-existence of multiple competing platforms (the firm and the network). In addition, we find that the equilibrium price moves in the opposite direction of the cost factor of downloading in case the two platforms co-exist. Although, in general, the price behaves non-monotonically when the cost factor of downloading becomes smaller, the profit unambiguously decreases. The total welfare, on the other hand, unambiguously increases.

\subsection{Appendix}

\subsubsection{The fully-served multi-platformed market}

The consumer $z \in(0,1)$ that separates the consumers choosing for the firm from those choosing for the network, is herself indifferent between the two options. Moreover this consumer should weakly prefer the firm to the option not to acquire the product. So, for a second-stage equilibrium $(p, d)$ to generate the structure $[S / N]$, it should hold that

$0 \leq U^{z}\left(p, d^{-z}, S\right)=U^{z}\left(p, d^{-z}, N\right) \Longleftrightarrow 0 \leq \beta-\tau z-p=\gamma-\tau(1-z)-\sigma(1-n(p, d))^{2}$,

where $n(p, d)=1-z$, since the market is fully-served. Solving the inequality for $z$ gives us two solutions:

$$
z_{c . m .}(p)=\frac{\tau+\sqrt{\sigma(p+\gamma-\beta-\tau)+\tau^{2}}}{\sigma} \quad \text { and } \quad z_{m . n .}(p)=\frac{\tau-\sqrt{\sigma(p+\gamma-\beta-\tau)+\tau^{2}}}{\sigma},
$$


where we call the solution corresponding to the smaller network size $\left(z_{c . m .}\right)$ the critical-mass network, and the solution corresponding to the larger network size $\left(z_{m . n .}\right)$ the maximum network. In order to guarantee the solution to be real, we need

$$
p \geq \beta-\gamma+\tau-\frac{\tau^{2}}{\sigma} .
$$

For the market structure $[S / N]_{*}$ to exist, the other conditions needed are:

$$
0 \leq z_{*}(p)<1 \quad \text { and } \quad \beta-\tau z_{*}(p)-p \geq 0 .
$$

The next two subsections deal with the maximum network and the criticalmass network, respectively.

\section{The maximum network}

Assume Inequality (2.1) holds. The maximum network solution exists for the multi-platformed and fully-served market if and only if the following two conditions are satisfied

$$
0 \leq \frac{\tau-\sqrt{\sigma(p+\gamma-\beta-\tau)+\tau^{2}}}{\sigma}<1 \quad \text { and } \quad \beta-\tau \frac{\tau-\sqrt{\sigma(p+\gamma-\beta-\tau)+\tau^{2}}}{\sigma}-p \geq 0 .
$$

The first condition is equivalent to

$$
p \leq \beta-\gamma+\tau \quad \text { and } \quad \sqrt{\sigma(p+\gamma-\beta-\tau)+\tau^{2}}>\tau-\sigma,
$$

where the second inequality is trivially satisfied owing to the assumption that $\sigma>\tau$ and using Inequality (2.1). The second condition is equivalent to

$$
\sqrt{\sigma(p+\gamma-\beta-\tau)+\tau^{2}} \geq \tau-\frac{\sigma}{\tau}(\beta-p) .
$$

We will argue next that the second condition is always satisfied. In case the right-hand side of the inequality above is negative, i.e. in case $p<\beta-$ $\tau^{2} / \sigma$, the inequality is trivially satisfied. Otherwise, $p \geq \beta-\tau^{2} / \sigma$, and the inequality above implies

$$
\frac{\sigma}{\tau^{2}}(\beta-p)^{2}-(\beta-p)-(\gamma-\tau) \leq 0,
$$

which is equivalent to

$$
\beta-\frac{\tau^{2}}{2 \sigma}\left(1+\sqrt{1+\frac{4 \sigma}{\tau^{2}}(\gamma-\tau)}\right) \leq p \leq \beta-\frac{\tau^{2}}{2 \sigma}\left(1-\sqrt{1+\frac{4 \sigma}{\tau^{2}}(\gamma-\tau)}\right) .
$$


Because $\gamma>\tau$, the lower bound on $p$ is less than $\beta-\tau^{2} / \sigma$ and therefore satisfied since we are considering the case $p \geq \beta-\tau^{2} / \sigma$. The upper bound is larger than $\beta$. Hence the second condition does not impose a further restriction on $p$.

Hence, Inequalities (2.1) and (2.2) boil down to

$$
\beta-\gamma+\tau-\frac{\tau^{2}}{\sigma} \leq p \leq \beta-\gamma+\tau .
$$

\section{The critical-mass network}

Assume Inequality (2.1) holds. The critical-mass network solution exists for the multi-platformed and fully-served market if and only if the following two conditions are satisfied

$$
0 \leq \frac{\tau+\sqrt{\sigma(p+\gamma-\beta-\tau)+\tau^{2}}}{\sigma}<1 \quad \text { and } \quad \beta-\tau \frac{\tau+\sqrt{\sigma(p+\gamma-\beta-\tau)+\tau^{2}}}{\sigma}-p \geq 0,
$$

Notice that the first inequality of the first condition is trivially satisfied and that the second inequality of this condition can be simplified to

$$
p<\beta-\gamma-\tau+\sigma
$$

The second inequality is equivalent to

$$
\sqrt{\sigma(p+\gamma-\beta-\tau)+\tau^{2}} \leq \frac{\sigma}{\tau}(\beta-p)-\tau .
$$

This condition holds only if the right-hand side of this inequality is nonnegative, that is $p \leq \beta-\tau^{2} / \sigma$, and

$$
\frac{\sigma}{\tau^{2}}(\beta-p)^{2}-(\beta-p)-(\gamma-\tau) \geq 0,
$$

where the latter inequality is equivalent to

$$
p \leq \beta-\frac{\tau^{2}}{2 \sigma}\left(1+\sqrt{1+\frac{4 \sigma}{\tau^{2}}(\gamma-\tau)}\right) \quad \text { or } \quad p \geq \beta-\frac{\tau^{2}}{2 \sigma}\left(1-\sqrt{1+\frac{4 \sigma}{\tau^{2}}(\gamma-\tau)}\right) .
$$

Since $\gamma>\tau$, the right-hand side of the second inequality exceeds $\beta$ and hence cannot be satisfied-leaving the first inequality to be satisfied. Moreover, the right-hand side of the first inequality is less than $\beta-\tau^{2} / \sigma$, hence the second condition is satisfied if and only if

$$
p \leq \beta-\frac{\tau^{2}}{2 \sigma}\left(1+\sqrt{1+\frac{4 \sigma}{\tau^{2}}(\gamma-\tau)}\right) .
$$


Next we show that the second condition is more restrictive than the first condition. Suppose the opposite holds true. That is, suppose that

$$
\beta-\frac{\tau^{2}}{2 \sigma}\left(1+\sqrt{1+\frac{4 \sigma}{\tau^{2}}(\gamma-\tau)}\right)>\beta-\gamma-\tau+\sigma .
$$

We will derive a contradiction. The supposition implies

$$
\sqrt{1+\frac{4 \sigma}{\tau^{2}}(\gamma-\tau)}<\frac{2 \sigma}{\tau^{2}}(\gamma+\tau-\sigma)-1
$$

For Inequality (2.4) to be satisfied, the right-hand side has to be positive, which is the case when

$$
2 \sigma(\gamma+\tau-\sigma)-\tau^{2}>0
$$

Inequality (2.4) then implies that

$$
\frac{\sigma}{\tau^{2}}(\gamma+\tau-\sigma)^{2}>2 \gamma-\sigma,
$$

which in turn implies that

$$
2 \tau^{2}+\sigma(\sigma-\gamma)-2 \tau \sigma>0
$$

We argue that Inequality (2.5) and (2.6) cannot be satisfied simultaneously. By adding Inequality (2.5) to twice Inequality (2.6) we find that $\tau>\frac{2}{3} \sigma$. Since we deal with two homogenous polynomial inequalities, we can set one of the parameters to any positive number. We set $\gamma=1$ and realize that this induces $\tau<1<\sigma$. Moreover, $\tau>\frac{2}{3} \sigma$ implies that $\sigma<\frac{3}{2}$. If we solve Inequality (2.6) for $\tau$, we find

$$
\tau<\frac{\sigma-\sqrt{2 \sigma-\sigma^{2}}}{2} \quad \text { or } \quad \tau>\frac{\sigma+\sqrt{2 \sigma-\sigma^{2}}}{2} .
$$

The first of these inequalities is in direct conflict with $\tau>\frac{2}{3} \sigma$, which leaves the second inequality as the only feasible option. However, for this inequality to hold true, $\left(\sigma+\sqrt{2 \sigma-\sigma^{2}}\right) / 2$ has to be less than 1 , so $\sigma$ has to be less than 1 or to be larger than 2 , which contradicts $\sigma \in\left(1, \frac{3}{2}\right)$. We have obtained a contradiction to the supposition. Hence,

$$
\beta-\frac{\tau^{2}}{2 \sigma}\left(1+\sqrt{1+\frac{4 \sigma}{\tau^{2}}(\gamma-\tau)}\right) \leq \beta-\gamma-\tau+\sigma .
$$

Hence, Inequalities (2.1) and (2.3) boil down to

$$
\beta-\gamma+\tau-\frac{\tau^{2}}{\sigma} \leq p \leq \beta-\frac{\tau^{2}}{2 \sigma}\left(1+\sqrt{1+\frac{4 \sigma}{\tau^{2}}(\gamma-\tau)}\right) .
$$




\subsubsection{The partially-served multi-platformed market}

The consumer $z^{S} \in(0,1)\left(z^{N} \in(0,1)\right)$ that separates the consumers choosing for the firm (network) from those choosing not to acquire any product, is herself indifferent between the two options. Moreover, this consumer prefers both the firm (network) and no acquisition to the option to acquire via the network (firm). We have a partially-served market if there is an interval of consumers that prefers no acquisition to acquisition, meaning that $z^{S}$ is less than $z^{N}$. So, for a second-stage equilibrium $(p, d)$ to generate the structure $[S / \varnothing / N]$, it should hold that

$$
U^{z^{S}}\left(p, d^{-z^{S}}, S\right)=U^{z^{S}}\left(p, d^{-z^{S}}, \varnothing\right) \text { and } U^{z^{N}}\left(p, d^{-z^{N}}, N\right)=U^{z^{N}}\left(p, d^{-z^{N}}, \varnothing\right)
$$

and

$$
0 \leq z^{S}(p)<z^{N}(p) \leq 1 .
$$

The first two equations are equivalent to

$$
\beta-\tau z^{S}-p=0 \quad \text { and } \quad \gamma-\tau\left(1-z^{N}\right)-\sigma(1-n(p, d))^{2}=0 .
$$

where $n(p, d)=1-z^{N}$. Solving the first equation for $z^{S}$ and the latter for $z^{N}$ gives us one solution for $z^{S}$ :

$$
z^{S}(p)=\frac{\beta-p}{\tau},
$$

and two solutions for $z^{N}$ :

$$
z_{c . m .}^{N}=\frac{\tau+\sqrt{\tau^{2}+4 \sigma(\gamma-\tau)}}{2 \sigma} \quad \text { and } \quad z_{m . n .}^{N}=\frac{\tau-\sqrt{\tau^{2}+4 \sigma(\gamma-\tau)}}{2 \sigma} .
$$

\section{The maximum network}

It is easily seen that $z_{m . n}^{N}$. is negative, so the maximum network solution cannot co-exist with a partially-served multi-platformed market.

\section{The critical-mass network}

The critical-mass network solution exists for the multi-platformed, partiallyserved market if and only if

$$
0 \leq \frac{\beta-p}{\tau}<\frac{\tau+\sqrt{\tau^{2}+4 \sigma(\gamma-\tau)}}{2 \sigma} \leq 1 .
$$


The first inequality is satisfied, since $p \leq \beta$. Owing to the assumption that $\sigma>\gamma$, the third inequality is satisfied too. Indeed,

$$
\frac{\tau+\sqrt{\tau^{2}+4 \sigma(\gamma-\tau)}}{2 \sigma}<1 \Longleftrightarrow \sqrt{\tau^{2}+4 \sigma(\gamma-\tau)}<2 \sigma-\tau \quad \Longleftrightarrow \quad \gamma<\sigma .
$$

The second inequality is equivalent to

$$
p>\beta-\frac{\tau^{2}}{2 \sigma}\left(1+\sqrt{1+\frac{4 \sigma}{\tau^{2}}(\gamma-\tau)}\right) .
$$

Hence the conditions in Inequality (2.7) are satisfied if and only if

$$
\beta-\frac{\tau^{2}}{2 \sigma}\left(1+\sqrt{1+\frac{4 \sigma}{\tau^{2}}(\gamma-\tau)}\right)<p .
$$

\subsubsection{Support for Proposition 2.5.1-2.5.3}

In this appendix we support our comparative statics results in Section 2.5. For each of the five different scenarios we provide the respective equilibrium values. Once a sign of the derivative of these values with respect to one of the model's parameters is not straightforward (that is, if the factor appears in multiple terms in possibly opposite directions), we provide the precise derivations.

Monopoly pricing \& fully-served market

\begin{tabular}{llllll}
\hline & & $\beta$ & $\gamma$ & $\tau$ & $\sigma$ \\
\hline$p^{*}$ & $=\beta-\tau$ & + & 0 & - & 0 \\
$s^{*}$ & $=1$ & 0 & 0 & 0 & 0 \\
$n^{*}$ & $=0$ & 0 & 0 & 0 & 0 \\
$\pi^{*}$ & $=\beta-\tau$ & + & 0 & - & 0 \\
$C S^{*}$ & $=\frac{1}{2} \tau$ & 0 & 0 & + & 0 \\
$W^{*}$ & $=\beta-\frac{1}{2} \tau$ & + & 0 & - & 0 \\
\hline
\end{tabular}

Monopoly pricing \& partially-served market

\begin{tabular}{llllll}
\hline & & $\beta$ & $\gamma$ & $\tau$ & $\sigma$ \\
\hline$p^{*}$ & $=\frac{1}{2} \beta$ & + & 0 & 0 & 0 \\
$s^{*}$ & $=\frac{1}{2 \tau} \beta$ & + & 0 & - & 0 \\
$n^{*}$ & $=0$ & 0 & 0 & 0 & 0 \\
$\pi^{*}$ & $=\frac{1}{4 \tau} \beta^{2}$ & + & 0 & - & 0 \\
$C S^{*}$ & $=\frac{1}{8 \tau} \beta^{2}$ & + & 0 & - & 0 \\
$W^{*}$ & $=\frac{3}{8 \tau} \beta^{2}$ & + & 0 & - & 0 \\
\hline
\end{tabular}


Network-deterring pricing \& fully-served market

\begin{tabular}{lllccc}
\hline & & $\beta$ & $\gamma$ & $\tau$ & $\sigma$ \\
\hline$p^{*}$ & $=\beta-\gamma+\tau-\frac{\tau^{2}}{\sigma}$ & + & - & $+/-^{1}$ & + \\
$s^{*}$ & $=1$ & 0 & 0 & 0 & 0 \\
$n^{*}$ & $=0$ & 0 & 0 & 0 & 0 \\
$\pi^{*}$ & $=\beta-\gamma+\tau-\frac{\tau^{2}}{\sigma}$ & + & - & $+/-^{1}$ & + \\
$C S^{*}$ & $=\gamma-\frac{3}{2} \tau+\frac{\tau^{2}}{\sigma}$ & 0 & + & $+/-^{2}$ & - \\
$W^{*}$ & $=\beta-\frac{1}{2} \tau$ & + & 0 & - & 0 \\
\hline
\end{tabular}

1. $\operatorname{sign}\left(\frac{\partial p^{*}}{\partial \tau}\right)=\operatorname{sign}\left(\frac{\partial \pi^{*}}{\partial \tau}\right)=\operatorname{sign}\left(1-\frac{2 \tau}{\sigma}\right)=\operatorname{sign}(\sigma-2 \tau)$. We have found parameter values compatible with the conditions in Proposition 2.4.4 that support a positive sign as well as a negative one.

2. $\operatorname{sign}\left(\frac{\partial C S^{*}}{\partial \tau}\right)=\operatorname{sign}\left(-\frac{3}{2}+\frac{2 \tau}{\sigma}\right)=\operatorname{sign}\left(\frac{4}{3} \tau-\sigma\right)$. As in 1 ., both positive and negative signs are found.

Network-deterring pricing \& partially-served market

\begin{tabular}{lllccc}
\hline & & $\beta$ & $\gamma$ & $\tau$ & $\sigma$ \\
\hline$p^{*}$ & $=\beta-\gamma+\tau-\frac{\tau^{2}}{\sigma}$ & + & - & $+/-^{1}$ & + \\
$s^{*}$ & $=\frac{1}{\tau}\left(\gamma-\tau+\frac{\tau^{2}}{\sigma}\right)$ & 0 & + & -2 & - \\
$n^{*}$ & $=0$ & 0 & 0 & 0 & 0 \\
$\pi^{*}$ & $=\left(\beta-\gamma+\tau-\frac{\tau^{2}}{\sigma}\right) \frac{1}{\tau}\left(\gamma-\tau+\frac{\tau^{2}}{\sigma}\right)$ & + & -3 & -4 & $+^{5}$ \\
$C S^{*}$ & $=\frac{1}{2 \tau}\left(\gamma-\tau+\frac{\tau^{2}}{\sigma}\right)^{2}$ & 0 & + & $+/-^{6}$ & - \\
$W^{*}$ & $=\left(\beta-\frac{1}{2}\left(\gamma-\tau+\frac{\tau^{2}}{\sigma}\right)\right) \frac{1}{\tau}\left(\gamma-\tau+\frac{\tau^{2}}{\sigma}\right)$ & + & $+^{7}$ & -8 & $-^{9}$ \\
\hline
\end{tabular}

1. $\operatorname{sign}\left(\frac{\partial p^{*}}{\partial \tau}\right)=\operatorname{sign}\left(1-\frac{2 \tau}{\sigma}\right)=\operatorname{sign}(\sigma-2 \tau)$. We have found parameter values compatible with the conditions in Proposition 2.4.4 that support a positive sign as well as a negative one.

2. $\frac{\partial s^{*}}{\partial \tau}=-\frac{\gamma}{\tau^{2}}+\frac{1}{\sigma}<0$, since $\gamma \sigma>\tau^{2}$.

3. $\frac{\partial \pi^{*}}{\partial \gamma}=\frac{1}{\tau}\left(\beta-2\left(\gamma-\tau+\frac{\tau^{2}}{\sigma}\right)\right)<0$, since one of the conditions in Proposition 2.4.4 is $\beta \leq 2\left(\gamma-\tau+\frac{\tau^{2}}{\sigma}\right)$.

4. $\frac{\partial \pi^{*}}{\partial \tau}=\ldots=-\frac{1}{\tau^{2}}\left(\beta-\gamma+\frac{\tau^{2}}{\sigma}\right)\left(\gamma-\tau+\frac{\tau^{2}}{\sigma}\right)+\frac{1}{\tau^{2}}\left(\beta-\gamma+\tau-\frac{\tau^{2}}{\sigma}\right)\left(-\tau+2 \frac{\tau^{2}}{\sigma}\right)=\ldots=$ $-\left\{\frac{1}{\tau^{2}}(\beta-\gamma) \gamma+\frac{1}{\sigma}\left[2\left(\gamma-\tau+\frac{\tau^{2}}{\sigma}\right)-\beta\right]+\left(\frac{\tau}{\sigma}-1\right)^{2}\right\}<0$, since $\beta>\gamma$ and $\beta<$ $2\left(\gamma-\tau+\frac{\tau^{2}}{\sigma}\right)$. 
5. $\frac{\partial \pi^{*}}{\partial \sigma}=\frac{\tau}{\sigma^{2}}\left(2\left(\gamma-\tau+\frac{\tau^{2}}{\sigma}\right)-\beta\right)>0$, again, using the condition in Proposition 2.4.4.

6. We have found parameter values compatible with the conditions in Proposition 2.4.4 that support a positive sign as well as a negative one.

7. $\frac{\partial W^{*}}{\partial \gamma}=\frac{1}{\tau}\left(\beta-\gamma+\tau-\frac{\tau^{2}}{\sigma}\right)>0$.

8. As a result of an increase in $\tau$, the demand curve shifts inward. Moreover, the number of consumers decreases as a result of the increase in $\tau$. Both effects induce a decrease in welfare. In formulas,

$$
W=\int_{x=0}^{\frac{1}{\tau}\left(\gamma-\tau+\frac{\tau^{2}}{\sigma}\right)}(\beta-\tau x) d x .
$$

As $\tau$ increases, $\frac{1}{\tau}\left(\gamma-\tau+\frac{\tau^{2}}{\sigma}\right)$ decreases, and for each $x, \beta-\tau x$ decreases. Since for each $x$ in the domain of integration $\beta-\tau x$ is positive, it follows that $W$ decreases as $\tau$ increases.

9. $\frac{\partial W^{*}}{\partial \sigma}=-\frac{\tau}{\sigma^{2}}\left(\beta-\gamma+\tau-\frac{\tau^{2}}{\sigma}\right)<0$.

\section{Co-existence}

\begin{tabular}{|c|c|c|c|c|c|}
\hline & & $\beta$ & $\gamma$ & $\tau$ & $\sigma$ \\
\hline$p^{*}$ & $=\frac{2}{9 \sigma}(2 \tau-\sqrt{\delta})(\tau+\sqrt{\delta})$ & $+^{1}$ & -2 & $t^{3}$ & $-{ }^{4}$ \\
\hline$s^{*}$ & $=\frac{1}{3 \sigma}(2 \tau-\sqrt{\delta})$ & + & - & -5 & $+^{6}$ \\
\hline$n^{*}$ & $=1-\frac{1}{3 \sigma}(2 \tau-\sqrt{\delta})$ & - & + & + & - \\
\hline$\pi^{*}$ & $=\frac{2}{27 \sigma^{2}}(2 \tau-\sqrt{\delta})^{2}(\tau+\sqrt{\delta})$ & $+{ }^{7}$ & -8 & $++^{9}$ & $++^{10}$ \\
\hline$C S^{*}$ & $=\gamma-\frac{1}{2} \tau-\frac{1}{9 \sigma^{2}}(2 \tau-\sqrt{\delta})^{2}(\sigma-\tau)$ & - & + & -11 & -12 \\
\hline$W^{*}$ & $=\gamma-\frac{1}{2} \tau-\frac{1}{27 \sigma^{2}}(2 \tau-\sqrt{\delta})^{2}(3 \sigma-5 \tau-2 \sqrt{\delta})$ & $++^{13}$ & $++^{14}$ & -15 & -16 \\
\hline
\end{tabular}

Notice that $C S^{*}=\left[\beta-\frac{1}{2} \tau s^{*}-p^{*}\right] s^{*}+\left[\gamma-\frac{1}{2} \tau n^{*}-\sigma\left(1-n^{*}\right)^{2}\right] n^{*}$.

Claim. The conditions in Proposition 2.4.5 imply $\sigma \in\left(\tau, \frac{4}{3} \tau\right)$ and $\sqrt{\delta} \in\left(\frac{1}{2} \tau, \tau\right)$.

Proof. The properties $\sigma \in\left(\tau, \frac{4}{3} \tau\right)$ and $\sqrt{\delta}<\tau$ follow directly from $\sigma, \beta>\gamma>\tau$ and $\delta \geq 0$. Left to be shown is $\sqrt{\delta}>\frac{1}{2} \tau$. First notice that

$$
\pi_{[S]}^{*}=\frac{1}{3 \sigma}(\tau+\sqrt{\delta})(\tau-\sqrt{\delta}) \text { and } \pi_{[S / \varnothing]}^{*}=\frac{1}{3 \sigma}(\tau+\sqrt{\delta})(\tau-\sqrt{\delta}) \frac{1}{\tau}\left(\gamma-\tau+\frac{\tau^{2}}{\sigma}\right) .
$$


Consider the case $\gamma \geq 2 \tau-\frac{\tau^{2}}{\sigma}$. Since $\pi^{*}>\pi_{[S]}^{*}$ we have

$$
2 \delta+(9 \sigma-8 \tau) \sqrt{\delta}-(9 \sigma-8 \tau) \tau>0
$$

Since this inequality is quadratic in $\sqrt{\delta}$ it is easily shown that it is satisfied if and only if

$$
\sqrt{\delta}>-\frac{1}{4}(9 \sigma-8 \tau)+\frac{1}{4} \sqrt{9 \sigma(9 \sigma-8 \tau)} .
$$

Since

$$
-\frac{1}{4}(9 \sigma-8 \tau)+\frac{1}{4} \sqrt{9 \sigma(9 \sigma-8 \tau)} \geq \frac{1}{2} \tau \Longleftrightarrow \sqrt{\sigma(9 \sigma-8 \tau)} \geq 3 \sigma-2 \tau \Longleftrightarrow \sigma \geq \tau
$$

we have shown that $\sqrt{\delta}>\frac{1}{2} \tau$.

Next, consider the case $\gamma<2 \tau-\frac{\tau^{2}}{\sigma}$. Since $\pi^{*}>\pi_{[S / \varnothing]}^{*}$ we have

$$
2 \delta+\frac{1}{\tau}\left(9 \sigma \gamma-9 \sigma \tau+\tau^{2}\right) \sqrt{\delta}-\left(9 \sigma \gamma-9 \sigma \tau+\tau^{2}\right)>0
$$

This inequality is satisfied if and only if

$$
\sqrt{\delta}>-\frac{1}{4 \tau}\left(9 \sigma \gamma-9 \sigma \tau+\tau^{2}\right)+\frac{1}{4 \tau} \sqrt{9\left(\sigma \gamma-\sigma \tau+\tau^{2}\right)\left(9 \sigma \gamma-9 \sigma \tau+\tau^{2}\right)}
$$

Since

$$
-\frac{1}{4 \tau}\left(9 \sigma \gamma-9 \sigma \tau+\tau^{2}\right)+\frac{1}{4 \tau} \sqrt{9\left(\sigma \gamma-\sigma \tau+\tau^{2}\right)\left(9 \sigma \gamma-9 \sigma \tau+\tau^{2}\right)} \geq \frac{1}{2} \tau
$$

if and only if

$$
\sqrt{\left(\sigma \gamma-\sigma \tau+\tau^{2}\right)\left(9 \sigma \gamma-9 \sigma \tau+\tau^{2}\right)} \geq 3 \sigma \gamma-3 \sigma \tau+\tau^{2}
$$

which is the case if and only if $\gamma \geq \tau$, we have shown that $\sqrt{\delta}>\frac{1}{2} \tau$.

1. $\frac{\partial p^{*}}{\partial \beta}=\frac{2}{9 \sigma}(2 \tau-\sqrt{\delta}) \frac{d \sqrt{\delta}}{d \beta}-\frac{2}{9 \sigma}(\tau+\sqrt{\delta}) \frac{d \sqrt{\delta}}{d \beta}=\frac{2}{9 \sigma}(\tau-2 \sqrt{\delta}) \frac{1}{2 \sqrt{\delta}}(-3 \sigma)=\frac{1}{3 \sqrt{\delta}}(2 \sqrt{\delta}-$ $\tau)>0$.

2. $\frac{\partial p^{*}}{\partial \gamma}=-\frac{\partial p^{*}}{\partial \beta}<0$.

3. $\frac{\partial p^{*}}{\partial \tau}=\frac{2}{9 \sigma}(2 \tau-\sqrt{\delta})\left(1+\frac{d \sqrt{\delta}}{d \tau}\right)+\frac{2}{9 \sigma}\left(2-\frac{d \sqrt{\delta}}{d \tau}\right)(\tau+\sqrt{\delta})=\frac{2}{9 \sigma}\left\{4 \tau+\sqrt{\delta}+\frac{d \sqrt{\delta}}{d \tau}(\tau-\right.$ $2 \sqrt{\delta})\}=\frac{2}{9 \sigma \sqrt{\delta}} \cdot\left\{4 \tau \sqrt{\delta}+\delta+\frac{1}{2}(8 \tau-3 \sigma)(\tau-2 \sqrt{\delta})\right\}=\frac{2}{9 \sigma} \frac{1}{\sqrt{\delta}}\{\delta+\sqrt{\delta}(3 \sigma-4 \tau)+$ $\left.\left(4 \tau^{2}-\frac{3}{2} \sigma \tau\right)\right\}>\frac{2}{9 \sigma} \frac{1}{\sqrt{\delta}}\left\{\delta-\tau^{2}+2 \tau^{2}\right\}>0$. 
4. Notice that $\frac{d \sqrt{\delta}}{d \sigma}=\frac{1}{2} \frac{1}{\sqrt{\delta}}[-3(\beta-\gamma+\tau)]=-\frac{1}{2} \frac{1}{\sigma} \frac{1}{\sqrt{\delta}}\left[4 \tau^{2}-\delta\right]=-\frac{1}{2} \frac{1}{\sigma} \frac{1}{\sqrt{\delta}}(2 \tau-$ $\sqrt{\delta})(2 \tau+\sqrt{\delta})$. Then $\frac{\partial p^{*}}{\partial \sigma}=\ldots=-\frac{2}{9 \sigma^{2}}(2 \tau-\sqrt{\delta})(\tau+\sqrt{\delta})-\frac{2}{9 \sigma^{2}}(\tau-2 \sqrt{\delta}) \frac{1}{2 \sqrt{\delta}}$. $(2 \tau-\sqrt{\delta})(2 \tau+\sqrt{\delta})=\ldots=-\frac{1}{9 \sigma^{2}}(2 \tau-\sqrt{\delta})^{2} \frac{1}{\sqrt{\delta}} \tau<0$.

5. $\frac{\partial s^{*}}{\partial \tau}=\frac{1}{3 \sigma} \frac{1}{\sqrt{\delta}}\left(2 \sqrt{\delta}-4 \tau+\frac{3}{2} \sigma\right)<\frac{1}{3 \sigma} \frac{1}{\sqrt{\delta}}\left(2 \sqrt{\delta}-4 \tau+\frac{3}{2} \frac{4}{3} \tau\right)=\frac{1}{3 \sigma} \frac{1}{\sqrt{\delta}} 2(\sqrt{\delta}-\tau)<0$.

6. $\frac{\partial s^{*}}{\partial \sigma}=\ldots=-\frac{1}{3 \sigma^{2}}(2 \tau-\sqrt{\delta})+\frac{1}{6 \sigma^{2}} \frac{1}{\sqrt{\delta}}(2 \tau-\sqrt{\delta})(2 \tau+\sqrt{\delta})=\frac{1}{6 \sigma^{2}} \frac{1}{\sqrt{\delta}}(2 \tau-\sqrt{\delta})^{2}>0$.

7. $\frac{\partial \pi^{*}}{\partial \beta}=\frac{2}{27 \sigma^{2}}\left\{2(2 \tau-\sqrt{\delta})\left(-\frac{d \sqrt{\delta}}{d \beta}\right)(\tau+\sqrt{\delta})+(2 \tau-\sqrt{\delta})^{2} \frac{d \sqrt{\delta}}{d \beta}\right\}=-\frac{2}{9 \sigma^{2}}(2 \tau-\sqrt{\delta}) \sqrt{\delta}$. $\frac{d \sqrt{\delta}}{d \beta}=\frac{1}{3 \sigma}(2 \tau-\sqrt{\delta})>0$.

8. $\frac{\partial \pi^{*}}{\partial \gamma}=-\frac{\partial \pi^{*}}{\partial \beta}<0$.

9. $\frac{\partial \pi^{*}}{\partial \tau}=\frac{2}{27 \sigma^{2}}(2 \tau-\sqrt{\delta})\left\{2(\tau+\sqrt{\delta})\left[2-\frac{d \sqrt{\delta}}{d \tau}\right]+(2 \tau-\sqrt{\delta})\left[1+\frac{d \sqrt{\delta}}{d \tau}\right]\right\}=\ldots=\frac{2}{9 \sigma^{2}}(2 \tau-$ $\sqrt{\delta})\left\{\frac{3}{2}(\sigma-\tau)+\frac{1}{2}(2 \sqrt{\delta}-\tau)\right\}>0$.

10. $\frac{\partial \pi^{*}}{\partial \sigma}=\frac{2}{27 \sigma^{2}}(2 \tau-\sqrt{\delta})\left\{-\frac{2}{\sigma}(2 \tau-\sqrt{\delta})(\tau+\sqrt{\delta})-2(\tau+\sqrt{\delta}) \frac{d \sqrt{\delta}}{d \sigma}+(2 \tau-\sqrt{\delta}) \frac{d \sqrt{\delta}}{d \sigma}\right\}=$ $\ldots($ see 4.$) \ldots=\frac{2}{27 \sigma^{2}}(2 \tau-\sqrt{\delta})\left\{-\frac{2}{\sigma}(2 \tau-\sqrt{\delta})(\tau+\sqrt{\delta})+\frac{3}{2 \sigma}(2 \tau-\sqrt{\delta})(2 \tau+\sqrt{\delta})\right\}=$ $\ldots=\frac{1}{27 \sigma^{3}}(2 \tau-\sqrt{\delta})^{3}>0$.

11. $\frac{\partial C S^{*}}{\partial \tau}=\frac{\partial W^{*}}{\partial \tau}-\frac{\partial \pi^{*}}{\partial \tau}<0$ (using 9. and 15.).

12. $\frac{\partial C S^{*}}{\partial \sigma}=\frac{\partial W^{*}}{\partial \sigma}-\frac{\partial \pi^{*}}{\partial \sigma}<0$ (using 10. and 16.).

13. $\frac{\partial W^{*}}{\partial \beta}=-\frac{1}{27 \sigma^{2}}(2 \tau-\sqrt{\delta})\left\{2\left(-\frac{d \sqrt{\delta}}{d \beta}\right)(3 \sigma-5 \tau-2 \sqrt{\delta})+(2 \tau-\sqrt{\delta})\left(-2 \frac{d \sqrt{\delta}}{d \beta}\right)\right\}=\ldots=$ $\frac{1}{3 \sigma}(2 \tau-\sqrt{\delta}) \frac{1}{\sqrt{\delta}}(\tau+\sqrt{\delta}-\sigma)>\frac{1}{3 \sigma}(2 \tau-\sqrt{\delta}) \frac{1}{\sqrt{\delta}} \frac{1}{6} \tau>0$.

14. $\frac{\partial W^{*}}{\partial \gamma}=1-\frac{1}{27 \sigma^{2}}(2 \tau-\sqrt{\delta})\left\{2\left(-\frac{d \sqrt{\delta}}{d \gamma}\right)(3 \sigma-5 \tau-2 \sqrt{\delta})+(2 \tau-\sqrt{\delta})\left(-2 \frac{d \sqrt{\delta}}{d \gamma}\right)\right\}=$ $\ldots=1-\frac{1}{3 \sigma}(2 \tau-\sqrt{\delta}) \frac{1}{\sqrt{\delta}}(\tau+\sqrt{\delta}-\sigma)=\frac{1}{3 \sigma} \frac{1}{\sqrt{\delta}}(\tau+\sqrt{\delta})[2(\sigma-\tau)+\sqrt{\delta}]>0$.

15. $\frac{\partial W^{*}}{\partial \tau}=\ldots=-\frac{1}{2}-\frac{1}{9}(2 \tau-\sqrt{\delta})\left\{\frac{1}{\sigma}-\frac{2 \tau}{\sigma^{2}}-\frac{\sqrt{\delta}}{\sigma^{2}}+\frac{8 \tau^{2}}{\sigma^{2} \sqrt{\delta}}-\frac{11 \tau}{\sigma \sqrt{\delta}}+\frac{3}{\sqrt{\delta}}\right\}$. First, let $\sigma=a \tau$ for $a \in\left(1, \frac{4}{3}\right)$. Then $\frac{\partial W^{*}}{\partial \tau}=-\frac{1}{2}-\frac{1}{9}(2 \tau-\sqrt{\delta})\left\{\frac{1}{a \tau}-\frac{2}{a^{2} \tau}-\frac{\sqrt{\delta}}{a^{2} \tau^{2}}+\frac{1}{\sqrt{\delta}}\left(\frac{8}{a^{2}}-\frac{11}{a}+3\right)\right\}$. Next, define $\alpha \equiv \frac{1}{a \tau}-\frac{2}{a^{2} \tau}-\frac{\sqrt{\delta}}{a^{2} \tau^{2}}+\frac{1}{\sqrt{\delta}}\left(\frac{8}{a^{2}}-\frac{11}{a}+3\right)$. If $\alpha \geq 0$ then clearly $\frac{\partial W^{*}}{\partial \tau}<0$. Suppose $\alpha<0$. Then $\alpha>\frac{1}{a \tau}-\frac{2}{a^{2} \tau}-\frac{\tau}{a^{2} \tau^{2}}+\frac{1}{\frac{1}{2} \tau}\left(\frac{8}{a^{2}}-\frac{11}{a}+3\right)=$ $\frac{1}{\tau}\left\{\frac{13}{a^{2}}-\frac{21}{a}+6\right\}>\frac{1}{\tau} \min _{a}\left\{\frac{13}{a^{2}}-\frac{21}{a}+6\right\}=-\frac{129}{52 \tau}$, where for the first inequality it has to be noted that $\frac{8}{a^{2}}-\frac{11}{a}+3<0$ for $a \in\left(1, \frac{4}{3}\right)$. Hence $\frac{\partial W^{*}}{\partial \tau}=-\frac{1}{2}-$ $\frac{1}{9}(2 \tau-\sqrt{\delta}) \cdot \alpha<-\frac{1}{2}-\frac{1}{9} \frac{3}{2} \tau \cdot\left(-\frac{129}{52 \tau}\right)=-\frac{9}{104}<0$. 
16. $\frac{\partial W^{*}}{\partial \sigma}=\frac{2}{27 \sigma^{3}}(2 \tau-\sqrt{\delta})^{2}(3 \sigma-5 \tau-2 \sqrt{\delta})-\frac{2}{27 \sigma^{2}}(2 \tau-\sqrt{\delta})\left(-\frac{d \sqrt{\delta}}{d \sigma}\right)(3 \sigma-5 \tau-$ $2 \sqrt{\delta})-\frac{1}{27 \sigma^{2}}(2 \tau-\sqrt{\delta})^{2}\left[3-2 \frac{d \sqrt{\delta}}{d \sigma}\right]=\ldots=\frac{1}{27 \sigma^{3}}(2 \tau-\sqrt{\delta})^{2} \frac{1}{\sqrt{\delta}}\{2 \sqrt{\delta}(3 \sigma-5 \tau-$ $2 \sqrt{\delta})-(2 \tau+\sqrt{\delta})(3 \sigma-5 \tau-2 \sqrt{\delta})-3 \sigma \sqrt{\delta}-(2 \tau-\sqrt{\delta})(2 \tau+\sqrt{\delta})\}=\ldots=-\frac{1}{27 \sigma^{3}}$. $(2 \tau-\sqrt{\delta})^{2} \frac{1}{\sqrt{\delta}}\{6 \tau(\sigma-\tau)+\sqrt{\delta}(\tau+\sqrt{\delta})\}<0$. 


\section{Chapter 3}

\section{Markets with a strong P2P network}

\subsection{Introduction}

This chapter ${ }^{1}$ studies a traditional monopolistic market of information goods in the presence of an inherently strong peer-to-peer file-sharing network. The focus here is to capture the case where there are always a few fanatic users who selflessly contribute to the sharing of files by uploading their own files (e.g. music, movies, software, etc.) for other's to download. This can happen, for instance, in a country where illegal file sharing is largely tolerated, where the P2P networks will gather users easily. It can also happen in a market with high quality digital form of the product, where the P2P networks will have more potential users than in a market with low quality digital copies. A review of relevant literature has been presented in Chapter 2 .

We adopt the same model as the one in the previous chapter from (Herings, Peeters, and Yang 2010), but with one crucial difference on the parameter assumptions. In Chapter 2, there is an assumption on two parameters that leads to the situation where no consumer will join a network if the size of such a network is expected to be 0 . We reverse the relation between these two parameters so that now the consumer who prefers the digital version the

\footnotetext{
${ }^{1}$ This chapter is an adapted version of the paper (Yang 2010).
} 
most will join a $\mathrm{P} 2 \mathrm{P}$ network even if the network is expected to be empty.

Furthermore, we relax the assumption on the relatively small parameter value of the consumers' taste heterogeneity. More specifically, we now allow consumers' taste heterogeneity to be so high that consumers with extreme preferences may not consume the least preferred version of the product even if it is costless. In so doing, we are able to capture more scenarios, including those where the market is partially served by the firm and the network together. We discover that coordination failure in the forming of networks is not an issue once we consider a high level of taste heterogeneity and include such fanatic users in the model.

In total, we find five possible equilibrium market structures in this chapter. Out of the five market structures, the most prevalent is the one where the firm accommodates the network and competes in price. In this case, the firm's profit suffers and the total welfare benefits from the existence of P2P. In the settings of this chapter, the firm can still deter the network, but under very limited circumstances when the taste heterogeneity is low, the quality of the physical form is high, and the legal enforcement is relatively strict. Think of the newspaper market as an example of this. The firm can also find itself in the position to want to optimally serve the whole market, if the value of its product is significantly higher than that of the pirated version, and taste heterogeneity is low. Another special market structure is that of a market-sharing case, which happens when taste heterogeneity is just so that the firm chooses to precisely serve the consumers who prefer not to download. Effectively, the firm lets the network form to its maximum size, and then serves the residual demand. Thus, the firm and the network together serve the market fully without competing with each other. Furthermore, there is a possibility where the firm can ignore the network and act as a monopoly, when taste heterogeneity is huge. Yet the firm's profit actually decreases in taste heterogeneity. So does the consumers' surplus. Total welfare, therefore, decreases in taste heterogeneity, and in the cost factor of downloading.

The remainder of the chapter is organized as follows. In Section 3.2 the two-stage model is described in detail. Next, the consumers' choices in the second stage, given the price set by the firm in the first stage, are presented in Section 3.3. Subsequently, in Section 3.4, the firm's pricing decision in 
the first stage and the resulting market structures are analyzed. Thereafter, Section 3.5 gives an overview of the equilibrium outcomes analyzed in Section 3.4 and provides economic intuitions. Section 3.6 discusses the comparative statics of the monopoly equilibrium outcome. Finally, Section 3.7 and 3.8 discuss the results and conclude.

\subsection{The model}

The model is taken from (Herings, Peeters, and Yang 2010), and it is a game with two stages. In stage one, the firm sets a price $p$ for the physical form of the product. Next, in stage two, after having observed the price set by the firm, consumers decide simultaneously and independently whether to purchase the physical form sold by the firm $(S)$, to download the digital form via the P2P network $(N)$, or not to acquire the product at all $(\varnothing)$. There is a continuum of consumers who differ in their relative preference of the physical form over the digital form.

The price $p$ set by the firm and the profile of consumers' choices determine the sales by the firm, $s \in[0,1]$, total quantity of consumers buying the physical form, and the size of the network $n \in[0,1]$, total quantity of consumers who choose to download the digital form from the network. The firm has zero costs in production and aims to maximize its profit, given by

$$
\pi=p \cdot s(p) .
$$

The utility of a consumer with identity $x \in[0,1]$ is given by

$$
U^{x}(p)= \begin{cases}\beta-\tau x-p & \text { if } S \\ \gamma-\tau(1-x)-C(n(p)) & \text { if } N \\ 0 & \text { if } \varnothing,\end{cases}
$$

where $\beta>0$ and $\gamma>0$ represent the basic utility of the physical and the digital form respectively. The identity $x \in[0,1]$ reflects the consumer's relative preference over the two forms. The consumer with identity $x=0$ has a strong preference for the physical form, whereas the consumer with identity $x=1$ has a strong preference for the digital form. For consumers $x \in(0,1)$, the acquisition of one of the forms generates a disutility that depends on the 
identity $x$ and the parameter $\tau>0$. The parameter $\tau$ captures the amount of heterogeneity in consumers' tastes. Finally, $C(n)$ represents the costs of downloading when the resulting network is of size $n \in[0,1]$.

$$
C(n)=\sigma \cdot(1-n)^{2},
$$

where $\sigma>0$ represents the generic cost factor of downloading, incorporating a collection of factors that may affect downloading costs, for instance, the degree of legal enforcement of intellectual property rights.

The main differences from Chapter 2 are in the following assumptions on the relevant parameters.

Assumption 3.2.1. $\beta>\gamma$.

Personal preferences aside, the objective product quality of the original physical form is higher than that of the digital form (which is "ripped" from the original).

Assumption 3.2.2. $\gamma>\sigma$.

There is at least one consumer (namely, the one located at $x=1$ ), who prefers joining P2P to not consuming at all even if the network is empty. Or to put it differently, the consumer who prefers the digital form the most is always willing to start a network. This implies that there is always a P2P network, unless the firm charges such a low price that even the consumer located at $x=1$ prefers to buy the physical form, in which case the market is fully served by the firm. In other words, the consumer located at $x=1$ is always served.

Note that we do not impose any assumptions on the value of $\tau$.

\subsection{Consumers' choice}

We start the analysis with determining the possible Nash equilibria for each of the subgames. Then, we consider the firm's pricing behavior in the first stage and analyze some interesting subgame-perfect Nash equilibrium outcomes.

As explained in Chapter 2, there are in total three equilibrium network sizes in the second stage of the game. The maximum network and the zero 
network are stable, and the critical-mass network is instable. This gives rise to the concept of coordination failure in network forming. Within our terminology, a network may experience coordination failure only when both the maximum and the critical-mass network exist, given the relevant price. In other words, if for a given price, the critical-mass network does not exist, coordination failure can not occur. In the majority part of the analysis in this chapter, the maximum network is the only equilibrium network size. A formal computation of the solutions of the critical-mass and maximum network in the fully- and partially-served markets can be found in Appendix 3.9.1.

Second-stage equilibrium market structures can differ in two dimensions: the degree to which consumers are served and the platforms that are actively used. Regarding the first dimension, the market can be fully served or partially served as is displayed in Figure 3.1 for a multi-platformed market. Regarding the second dimension we can have a multi-platformed mar-
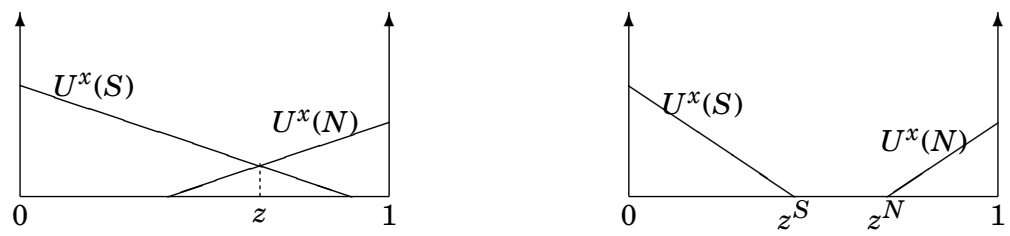

Figure 3.1: Fully-served market and partially-served market.

ket or a single-platformed market with either only the firm or only the P2P network. Note that the market can not be partially served by the store alone due to Assumption 3.2.2, i.e. [S/ $\varnothing]$ does not exist. For the second stage, we can therefore restrict our attention to the five market structures depicted in Table 3.1. Observe that, the situation in the graph on the left of Figure 3.1 is denoted by $[S / N]$; and the one on the right by $[S / \varnothing / N]$.

\begin{tabular}{lccc}
\hline & \multicolumn{2}{c}{ single-platformed } & \\
\cline { 2 - 3 } & firm & network & multi-platformed \\
\hline fully-served & {$[S]$} & {$[N]$} & {$[S / N]$} \\
partially-served & & {$[\varnothing / N]$} & {$[S / \varnothing / N]$} \\
\hline
\end{tabular}

Table 3.1: All possible market structures in stage two. 
Which one of these five possible market structures prevails in the second stage depends, apart from the price $p$ set in the first stage, on the parameter values. The set of parameter values determines the group of possible secondstage equilibrium market structures.

Recalling Assumption 3.2.2, we have $\gamma>\sigma$, but no restrictions on their relations with $\tau$. Not putting any assumptions on $\tau$ results in three possible scenarios: high heterogeneity scenario $(\tau>\gamma>\sigma)$, medium heterogeneity scenario $(\gamma>\tau>\sigma)$, and low heterogeneity scenario $(\gamma>\sigma>\tau){ }^{2}$ These three different scenarios in turn yield very different equilibrium market outcomes in the second stage given the price set by the firm in the first stage. Depending on the level of taste heterogeneity, the market can be fully or partially served, by single or multiple platforms. In the following analysis we will examine the scenarios separately .

Proposition 3.3.1. Given the price $p$ set in the first stage by the firm, all second-stage equilibrium market structures in the high heterogeneity scenario $(\tau>\gamma>\sigma)$ are as depicted in Figure 3.2.

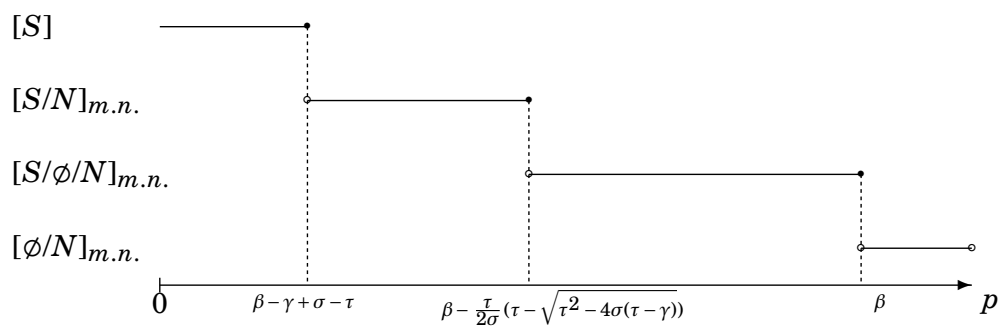

Figure 3.2: High heterogeneity scenario

The horizontal axis depicts the first-stage price that the firm charges, and the corresponding market structures are shown on the vertical axis. ${ }^{3}$

As the firm raises its price, it gradually loses its market share. When it charges a price lower than $\beta-\gamma+\sigma-\tau$, it fully serves the market alone and deters the formation of the network. Note that if $\beta-\gamma+\sigma-\tau<0$, the

\footnotetext{
${ }^{2}$ We ignore the specific analysis of boundary cases where $\tau=\gamma$ or $\tau=\sigma$, since they produce degenerate results without additional insights.

${ }^{3}$ The proofs of Propositions 3.3.1, 3.3.2 and 3.3.3 can be found in Appendix 3.9.2.
} 
market structure $[S]$ will disappear and $[S / N]$ will start at the price of 0 instead. But since this condition does not affect the equilibrium outcome, we shall not go through the trouble to treat it with separate scenarios. At a price between $\beta-\gamma+\sigma-\tau$ and $\beta-\tau\left(\tau-\sqrt{\tau^{2}-4 \sigma(\tau-\gamma)}\right) / 2 \sigma$, the firm accommodates the network and together they serve the market fully. At a price between $\beta-\tau\left(\tau-\sqrt{\tau^{2}-4 \sigma(\tau-\gamma)}\right) / 2 \sigma$ and $\beta$, it ignores the network and acts as a monopoly to serve its own monopolistic market share. In this case the market is partially served. Again, if $\beta-\tau\left(\tau-\sqrt{\tau^{2}-4 \sigma(\tau-\gamma)}\right) / 2 \sigma<0$, both $[S]$ and $[S / N]$ will disappear from the graph, and $[S / \varnothing / N]$ will start at the price of 0 . And finally at a price higher than $\beta$, it can not manage to make any sales.

Notice that there is a unique second-stage equilibrium market structure for each first-stage price $p$. Specifically, in the price range where the network might form, it is only the maximum network and not the critical-mass network that exists. This is due to the fact that $\tau>\sigma$. The implication is that coordination failure never occurs. In the partially-served market $[S / \varnothing / N]$, this is caused by the parameter setting of Assumption 3.2.2, $\gamma>\sigma$. Since the firm is not directly involved at the digital end of the market (where $x$ is close to 1 ), the fact that there are fanatic P2P users joining even if no one else joins helps eliminate the possibility of a potential coordination failure. On the other hand, in the fully-served market $[S / N]$, the lack of coordination failure is caused by the parameter setting within this scenario, $\tau>\sigma$. When the degree of taste heterogeneity is high relative to the cost factor of downloading and the market is still fully served, it must be that the network is inherently so strong that the required size of a critical-mass network is extremely low (indeed negative) and therefore always fulfilled.

The existence of the partially-served, multi-platformed market structure $[S / \varnothing / N]$ is also caused by the parameter setting $\tau>\sigma$. The consumers are sufficiently differentiated such that a section of the market in the middle can be left unserved in equilibrium. Furthermore, the fact that $\gamma>\sigma$ leads to the direct result that the digital end of the market is always served, leaving the market structure $[S / \varnothing]$ an impossibility.

In order to determine which price the firm will actually choose in stage one, one needs to investigate the profit levels of these different market structures in stage one. This will be dealt with in the next section, and we con- 
tinue this section with analyzing the possible equilibrium market structures that can result from the firm's pricing in the other two scenarios.

Proposition 3.3.2. Given the price $p$ set in the first stage by the firm, all second-stage equilibrium market structures in the medium heterogeneity scenario $(\gamma>\tau>\sigma)$ are as depicted in Figure 3.3.

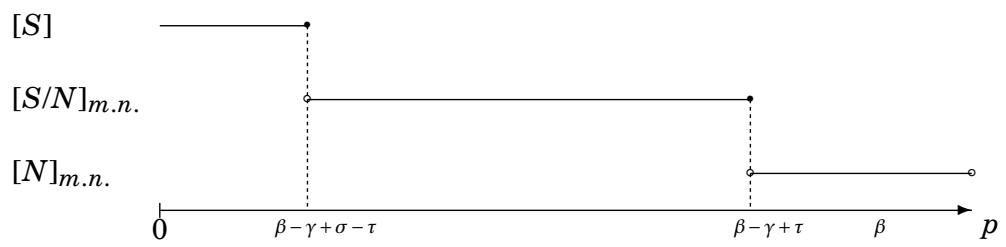

Figure 3.3: Medium heterogeneity scenario

Similarly to Proposition 3.3.1, there is a unique second-stage equilibrium market structure for each first-stage price $p$. Notice again in particular the lack of critical-mass network and coordination failure in network forming. This proposition also bares some other similarities with Proposition 3.3.1.

The firm is still able to serve the whole market here if it charges a price $p \leq \beta-\gamma+\sigma-\tau$. Again, if $\beta-\gamma+\sigma-\tau<0$, market structure $[S / N]$ will start at the price of 0 . The resulting market structure is then single-platformed fully-served market $[S]$. With a price any higher than that, a network will form and for prices $p \leq \beta-\gamma+\tau$ the resulting market structure will be $[S / N]$. Notice that, unlike in the high heterogeneity scenario (Proposition 3.3.1), the market structure $[S / \varnothing / N]$ does not exist in this scenario. This is the case because the consumers are not sufficiently differentiated for the firm to act like a local monopoly and partially serve the market. In other words, the firm is not able to ignore the network and act as a monopoly due to the lower level of taste heterogeneity $\tau$ in this scenario.

For all prices $p>\beta-\gamma+\tau$ the firm fails to make any sales and the market will be fully served by the network, hence the market structure $[N]$. Notice that this price is lower than $\beta$, which is the lower-bound price of the zerosales market structure $[\varnothing / N]$ in Proposition 3.3.1. This reflects the fact that, comparing to the high heterogeneity scenario, the firm is now facing less differentiated consumers and hence more direct competition from the network. 
It now has less freedom in price setting since it will lose all its consumers at an even lower price, comparing to the high heterogeneity scenario.

Proposition 3.3.3. Given the price $p$ set in the first stage by the firm, all second-stage equilibrium market structures in the low heterogeneity scenario $(\gamma>\sigma>\tau)$ are as depicted in Figure 3.4.

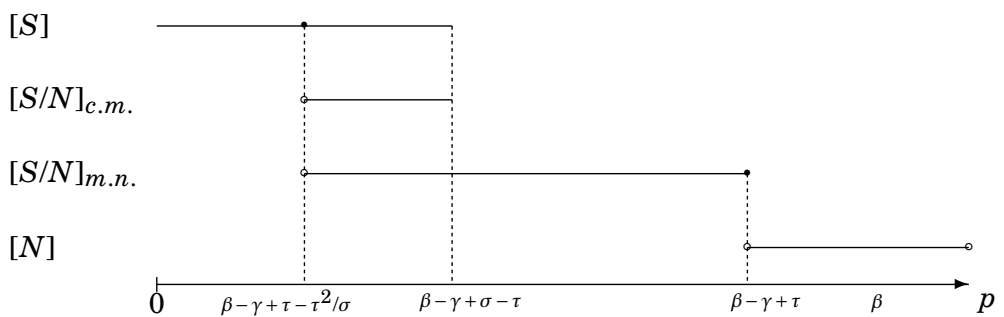

Figure 3.4: Low heterogeneity scenario.

The first striking difference here from the previous propositions is that there are multiple equilibria. Secondly, unlike in the two previous propositions, we find a critical-mass network equilibrium (see Appendix 3.9.2 for the proof). This implies that it is now possible to have a coordination failure in network forming, specifically within the price range $\left[\beta-\gamma+\tau-\tau^{2} / \sigma, \beta-\gamma+\sigma-\tau\right]$. When the firm charges a price in this range, it is possible that the consumers are able to form a network if everyone expects this to be the case, or fail to do so if everyone believes a network will not form.

The upper-bound price of $[S / N]_{m . n}$. is $\beta-\gamma+\tau$, as in Proposition 3.3.2. However, recall that in Proposition 3.3.1 and 3.3.2, where $\tau>\sigma$, the upperbound price for $[S]$ and the lower-bound price $[S / N]_{m . n}$. are the same. Here with $\tau<\sigma$, when solving for the condition that a network must have the size smaller than 1, the resulting inequality gives a smaller lower-bound price for the market structures $[S / N]_{c . m}$. and $[S / N]_{m . n}$. than the upper-bound price for the market structure $[S]$. Therefore, there is a price range where these two market structures overlap, namely, when $\beta-\gamma+\tau-\tau^{2} / \sigma<p<\beta-\gamma+\sigma-\tau$. This is also the price range where the critical-mass network exists. A crucial point here is that the firm's profit levels are not continuous when switching form $[S]$ to $[S / N]$ at the price $\beta-\gamma+\tau-\tau^{2} / \sigma$. The firm can still fully serve 
the market alone if it charges a price no higher than $\beta-\gamma+\tau-\tau^{2} / \sigma$, provided that it is positive.

Note that it must hold that $\beta-\gamma+\tau-\tau^{2} / \sigma>0$, otherwise all three market structures $[S],[S / N]_{c . m}$. and $[S / N]_{m . n}$. start at the price of 0 , and the section in the graph where $[S]$ is the unique market structure will not exist. Moreover, for the section in the graph where $[S / N]_{m . n}$. is the unique the market structure in the price range $[\beta-\gamma+\sigma-\tau, \beta-\gamma+\tau]$, it must hold that $\beta-\gamma+\tau>\beta-\gamma+\sigma-\tau$, which means $\sigma<2 \tau$. Otherwise, all three market structures $[S],[S / N]_{c . m}$. and $[S / N]_{m . n}$. will end at the price of $p=\beta-\gamma+\tau$, where the market structure $[N]$ starts.

\subsection{Firm's decision}

The firm sets a profit maximizing price $p$ in stage one, taking into account the possible realized equilibrium market structure in stage two. Depending on the different scenarios in stage two we discussed in the previous section, the optimum price $p$ can vary in stage one.

In this section, we investigate subgame-perfect equilibrium prices and market outcomes for each of the scenarios analyzed in the previous section. In the next section, we will then summarize and regroup the findings with respect to the equilibrium market structures in order to provide more economic insights and intuitions.

In the low heterogeneity scenario $(\gamma>\sigma>\tau)$, the possible equilibrium market structures in stage two involve multiple equilibria. To deal with the multiplicity of subgame-perfect equilibria, we follow the convention by supposing that, once the price is known, consumers coordinate on the equilibrium continuation that they prefer, which is the one with the largest network size. $^{4}$

Similar to Chapter 2, we adopt the approach where the second-stage equilibrium market structure that corresponds to the firm-worst response of the consumers, given the first-stage price $p$, is captured by a lower envelop. This is depicted in Figure 3.5 below. The lower envelop is the collection of the three solid lines. Notice that within the price range $\left[\beta-\gamma+\tau-\tau^{2} / \sigma, \beta-\right.$

\footnotetext{
${ }^{4}$ See, for instance, (Katz and Shapiro 1986) or (Fudenberg and Tirole 2000).
} 
$\gamma+\sigma-\tau]$, where multiple second-stage equilibria occur, only the one with the lowest profit for the firm, i.e. $[S / N]_{m . n .}$, is on the lower envelop. Here, we do not try to produce a full characterization of all subgame-perfect equilibria. ${ }^{5}$ We will concentrate instead on the equilibrium continuation that consumers prefer the most, i.e. the one with the largest network, and assume that they coordinate on this equilibrium continuation after the price is known. This in fact coincides with the firm-worst situation represented by the lower envelop.

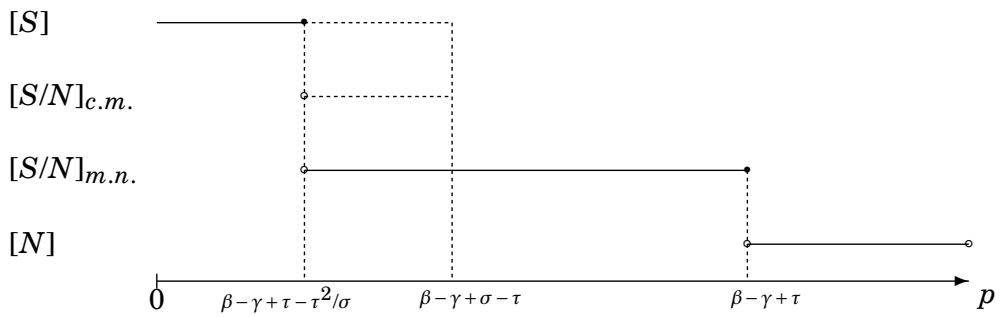

Figure 3.5: The lower envelop of the low heterogeneity scenario.

For the convenience in notation, we define $\delta \equiv 4 \tau^{2}-3 \sigma(\beta-\gamma+\tau)$. Roughly speaking, $\delta$ represents the attractiveness of the network relative to the firm. Indeed, $\delta$ is increasing in $\gamma-\beta$ and decreasing in $\sigma$.

Furthermore, we use $\Pi_{[\cdot]}^{*}$ to denote the maximum profit of any market structure [·] as depicted in Table 3.1.

Proposition 3.4.1. In the high heterogeneity scenario $(\tau>\gamma>\sigma)$, there are parameter settings such that the following four market outcomes and their associated price and profit levels can be supported by a subgame-perfect equilibrium.

1. The firm serves the entire market by charging a price $p=\beta-\gamma+\sigma-\tau$, and makes a profit of $\Pi_{[S]}^{*}=\beta-\gamma+\sigma-\tau$.

2. The firm competes directly with the P2P network by charging a price $p=2(2 \tau-\sqrt{\delta})(\tau+\sqrt{\delta}) / 9 \sigma$ and earns a profit of $\Pi_{[S / N]}^{*}=2(2 \tau-\sqrt{\delta})^{2}(\tau+$ $\sqrt{\delta}) / 27 \sigma^{2}$.

\footnotetext{
${ }^{5}$ For a vigorous derivation of such a full characterization, see Chapter 2.
} 
3. The firm serves precisely up to the last consumer who does not download from P2P by charging a price $p=\beta-\tau\left(\tau-\sqrt{\tau^{2}-4 \sigma(\tau-\gamma)}\right) / 2 \sigma$, and earns a profit of $\Pi_{[S / N]-\text { boundary }}=\left(\tau-\sqrt{4 \gamma \sigma-4 \sigma \tau+\tau^{2}}\right)\left(2 \beta \sigma-\tau^{2}+\right.$ $\left.\tau \sqrt{4 \gamma \sigma-4 \sigma \tau+\tau^{2}}\right) / 4 \sigma^{2}$.

4. The firm acts as a local monopoly and charges the price $p=\beta / 2$ and earns a profit of $\Pi_{[S / \varnothing / N]}^{*}=\beta^{2} / 4 \tau$.

Proof.

It is helpful to look back at Figure 3.2. First of all, any price $p>\beta$ can not be profit-maximizing, since under the market structure of $[\varnothing / N]$ the firm's sales quantity and profit are invariably 0 , and the firm can always do better by charging a price that yields a positive sales quantity. We therefore conclude that this market structure can not be supported by any subgame-perfect equilibrium.

Secondly, any price $p<\beta-\gamma+\sigma-\tau$ can not be profit-maximizing, since the resulting sales quantity is always 1 (the entire market), and the profit function is continuous and monotonic in price. This makes $p=\beta-\gamma+\sigma-\tau$ strictly more profitable than any lower price. Therefore, the highest profit under the market structure $[S]$ corresponds to this price $p=\beta-\gamma+\sigma-\tau$. Moreover, the profit levels are continuous at this price where the market structure switches from $[S]$ to $[S / N]$. The first derivative of the profit function with respect to price under the market structure $[S / N]$ is not strictly positive under the condition $\beta-\gamma \geq 3(\tau-\sigma)$, which implies that the profit at this price can be a global maximum profit and hence may be supported by a subgame-perfect equilibrium. However, when $\beta-\gamma<3(\tau-\sigma)$, the profit levels at this price is both continuous and monotonic, in which case the profit at this price can not be a global maximum.

The profit levels under the market structure of $[S / N]$, can be non-monotonic with a local maximum value of $\Pi_{[S / N]}^{*}=2(2 \tau-\sqrt{\delta})^{2}(\tau+\sqrt{\delta}) / 27 \sigma^{2}$, at the price of $p_{[S / N]}^{*}=2(2 \tau-\sqrt{\delta})(\tau+\sqrt{\delta}) / 9 \sigma$, under the condition that $\delta \geq 0$. This profit level can be a global maximum, hence may be supported by a subgame-perfect equilibrium.

However, if $\delta<0$, the maximum profit of $[S / N]$ is achieved at the upper boundary price $p=\beta-\tau\left(\tau-\sqrt{\tau^{2}-4 \sigma(\tau-\gamma)}\right) / 2 \sigma$, yielding a profit level of $\Pi_{[S / N]-\text { boundary }}=\left(\tau-\sqrt{4 \gamma \sigma-4 \sigma \tau+\tau^{2}}\right)\left(2 \beta \sigma-\tau^{2}+\tau \sqrt{4 \gamma \sigma-4 \sigma \tau+\tau^{2}}\right) / 4 \sigma^{2}$. 
This can be supported by a subgame-perfect equilibrium only if the profit under the market structure $[S / \varnothing / N]$ at this price is decreasing and the profit function is strictly monotonic across its price range until its upper-bound price $p=\beta$.

Finally, the profit levels under the market structure of $[S / \varnothing / N]$, also can be non-monotonic. Under this market structure, the firm is acting as a monopoly and does not compete directly with the network. The consumer $x$ who is indifferent from buying the physical product in the store and not buying has utility level $\beta-\tau x-p=0$, which implies $x=(\beta-p) / \tau$. Taking this as a demand function and the firm optimizes its price at $p_{[S / \phi / N]}^{*}=\beta / 2$, resulting a profit level of $\Pi_{[S / \phi / N]}^{*}=\beta^{2} / 4 \tau$. For this interior solution to be the maximum profit in this price range, two conditions have to be met. The first one is that $x<1$, which implies that $\beta<2 \tau$ must hold. The second one requires that the price $p_{[S / \phi / N]}^{*}=\beta / 2$ to be in the price range of $[S / \varnothing / N]$, in particular, $\beta<\tau^{2}-\tau \sqrt{\tau^{2}-4 \sigma(\tau-\gamma)}$. This maximum profit can be a global maximum, hence may be supported by a subgame-perfect equilibrium.

In order to demonstrate the existence of parameter settings that qualify the above four numbered items to be subgame-perfect equilibrium outcomes, we provide for each of them a set of example parameters in Table 3.2. Each of the four columns of the table presents a set of parameter values that yield the corresponding profit level as the global maximum, in the order of the four bullets points described in Proposition 3.4.1.

\begin{tabular}{l|cccc}
\hline & \multicolumn{4}{|c}{ Equilibrium outcomes } \\
Parameters & $\Pi_{[S]}^{*}$ & $\Pi_{[S / N]}^{*}$ & $\Pi_{[S / N]}-$ boundary & $\Pi_{[S / \phi / N]}^{*}$ \\
\hline$\beta$ & 19 & 12 & 12 & 10 \\
$\gamma$ & 9 & 8 & 8 & 9 \\
$\sigma$ & 8 & 7 & 7 & 8 \\
$\tau$ & 10 & 10 & 12 & 14 \\
\hline
\end{tabular}

Table 3.2: Examples of parameter values for Proposition 3.4.1 (high heterogeneity scenario)

The relevant profit functions given these four sets of parameter values are as depicted in Figure 3.6. The four pictures represent the four sets of pa- 
rameter values depicted in Table 3.2 respectively. In each picture, the firm's profit level is depicted as a function of its price. Different line styles represent different market structures, which are labeled by the profit legends $\left(\Pi_{[S]}, \Pi_{[S / N]}, \ldots\right)$. The global maximum profits in these four pictures are achieved under the market structures indicated by the market legends at the bottom of each picture ([S], $[S / N], \ldots)$, as described in Proposition 3.4.1.

$\pi$

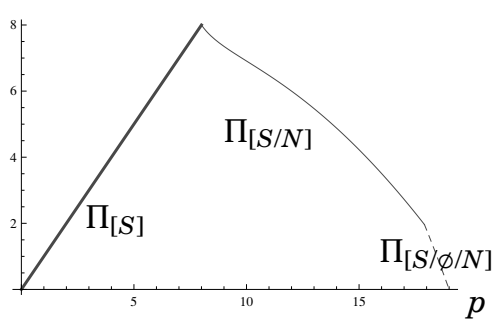

1. $[S]$

$\pi$

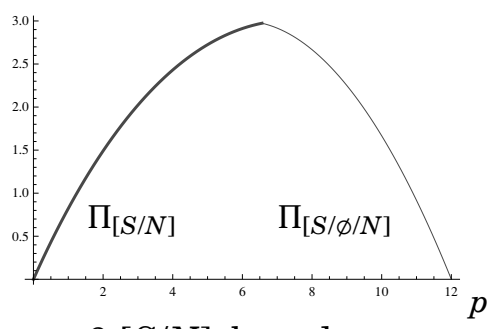

3. $[S / N]$-boundary $\pi$

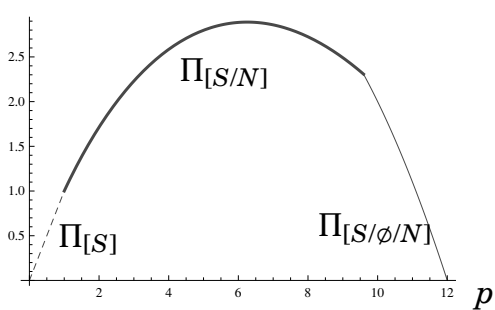

2. $[S / N]$

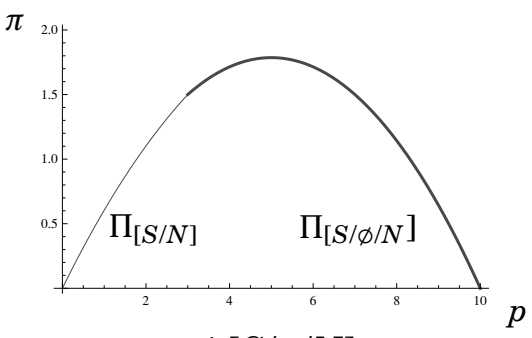

4. $[S / \varnothing / N]$

Figure 3.6: Profit functions and equilibrium outcomes (high heterogeneity scenario)

We only plot the profit levels in the relevant price range, namely, $p \in$ $[0, \beta]$. Notice the continuity of profit levels in all four pictures while rising and falling with prices across different market structures.

Proposition 3.4.2. In the medium heterogeneity scenario $(\gamma>\tau>\sigma)$, there are parameter settings such that the following two market outcomes and their associated price and profit levels can be supported by a subgameperfect equilibrium.

1. The firm serves the entire market by charging a price $p=\beta-\gamma+\sigma-\tau$, and makes a profit level of $\Pi_{[S]}^{*}=\beta-\gamma+\sigma-\tau$. 
2. The firm competes directly with the network by charging a price $p=$ $2(2 \tau-\sqrt{\delta})(\tau+\sqrt{\delta}) / 9 \sigma$ and earns a profit of $\Pi_{[S / N]}^{*}=2(2 \tau-\sqrt{\delta})^{2}(\tau+$ $\sqrt{\delta}) / 27 \sigma^{2}$.

Proof.

In this scenario, as Figure 3.3 shows, there are three possible equilibrium market structures in stage two given firm's price $p$ from stage one.

Similar to Proposition 3.4.1, the maximum profit under the market structure $[S]$ is achieved at the boundary price $p=\beta-\gamma+\sigma-\tau$. Under the condition that $\beta-\gamma \geq 3(\tau-\sigma)$, the first derivative of the profit function $\Pi_{[S / N]}$ at this price is non positive, which implies that this profit level can be a global maximum, hence may be supported by a subgame-perfect equilibrium.

If $\beta-\gamma<3(\tau-\sigma)$, the profit levels under the market structure [S] are strictly dominated by at least one profit level under the market structure $[S / N]$. In this case, The profit function $\Pi_{[S / N]}$ reaches a local maximum $\Pi_{[S / N]}^{*}=2(2 \tau-\sqrt{\delta})^{2}(\tau+\sqrt{\delta}) / 27 \sigma^{2}$, before subsequently decreasing until reaching 0 at the upper-bound price $p=\beta-\gamma+\tau$.

Finally, the market structure $[N]$ with the firm having zero profit can not be sustained by any subgame-perfect equilibrium either, since the firm can always do better by charging a lower price in order to have at least some sales.

Again we demonstrate the existence of parameter settings that qualify the above two numbered items to be subgame-perfect equilibrium outcomes by providing for each of them a set of example parameters in Table 3.3. Each of the two columns of Table 3.3 presents a set of parameter values that yield the corresponding profit level as the global maximum, in the order of the two numbered items described in Proposition 3.4.2.

The relevant profit functions given these two sets of parameter values are as depicted in Figure 3.7. The two pictures represent the two sets of parameter values depicted in Table 3.3 respectively. Notice once again the continuity in profit levels across market structures.

Proposition 3.4.3. In the low heterogeneity scenario $(\gamma>\sigma>\tau)$, there are parameter settings such that the following two market outcomes and their associated price and profit levels can be supported by a subgame-perfect equilibrium. 
Chapter 3. Markets with a strong P2P network

\begin{tabular}{l|cc}
\hline & \multicolumn{2}{|c}{ Equilibrium outcomes } \\
Parameters & $\Pi_{[S]}^{*}$ & $\Pi_{[S / N]}^{*}$ \\
\hline$\beta$ & 16 & 13 \\
$\gamma$ & 11 & 11 \\
$\sigma$ & 9 & 9 \\
$\tau$ & 10 & 10 \\
\hline
\end{tabular}

Table 3.3: Examples of parameter values for Proposition 3.4.2 (medium heterogeneity scenario)

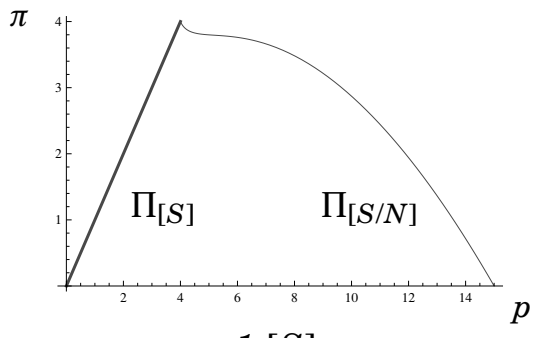

1. $[S]$

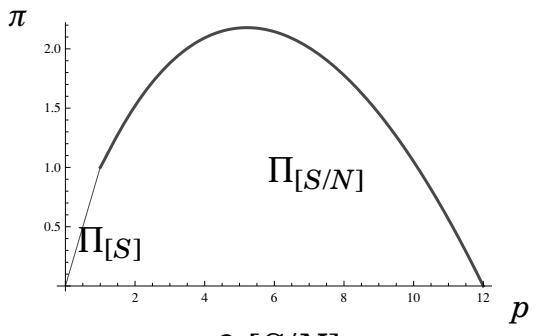

2. $[S / N]$

Figure 3.7: Profit functions and equilibrium outcomes (medium heterogeneity scenario)

1. The firm sets a network-deterring price $p=\beta-\gamma+\tau-\tau^{2} / \sigma$ and serves the entire market alone, making a profit of $\Pi_{[S]}^{*}=\beta-\gamma+\tau-\tau^{2} / \sigma$.

2. The firm competes directly with the network by charging a price $p=$ $2(2 \tau-\sqrt{\delta})(\tau+\sqrt{\delta}) / 9 \sigma$ and earns a profit of $\Pi_{[S / N]}^{*}=2(2 \tau-\sqrt{\delta})^{2}(\tau+$ $\sqrt{\delta}) / 27 \sigma^{2}$.

Proof.

It is helpful to look back at the lower envelop in Figure 3.5 at the beginning of the section.

First of all, as the previous propositions, the last part of the lower envelop representing the second stage equilibrium $[N]$ clearly can not yield any possible subgame-perfect equilibrium outcome, because the firm is making zero profit. However, the other two sections, namely $[S]$ and $[S / N]_{m . n}$, can.

The highest profit level on the $[S]$ section of the lower envelop is achieved 
at the right-hand extreme of the line at the price of $p_{[S]}^{*}=\beta-\gamma+\tau-\tau^{2} / \sigma$, under the condition that it is positive. Since sales quantity is 1 in a fullyserved market, this yields a profit of $\Pi_{[S]}^{*}=\beta-\gamma+\tau-\tau^{2} / \sigma$, which can be a global maximum profit and hence may be supported by a subgame-perfect equilibrium.

The profit function on the section $[S / N]_{m . n}$. is similar to the previous two propositions. However, observe the discontinuity of the profit levels from the market structure $[S]$ to $[S / N]_{m . n}$. at the price level $p=\beta-\gamma+\tau-\tau^{2} / \sigma$. In fact, the profit level at this price under the market structure $[S / N]_{m . n}$. is exactly a fraction $\frac{\tau}{\sigma}$ of the profit under the market structure of $[S]$. In other words, a small increase in price triggers a network of the size $1-\frac{\tau}{\sigma}$ to form and significantly hurts the sales quantity of the firm. The profit function $\Pi_{[S / N]_{m . n} \text {. }}$ does increase in price though, until reaching its maximum $\Pi_{[S / N]_{m . n}}^{*}=2(2 \tau-$ $\sqrt{\delta})^{2}(\tau+\sqrt{\delta}) / 27 \sigma^{2}$ at the price $p_{[S / N]}^{*}=2(2 \tau-\sqrt{\delta})(\tau+\sqrt{\delta}) / 9 \sigma$, before decreasing again all the way to zero, under the condition $\delta \geq 0$. This can be a global maximum profit and hence may be supported by a subgame-perfect equilibrium.

Notice that the maximum profit under the market structure $[S / N]$ is reached at its lower-bound price if $\delta<0$, which implies $\beta-\gamma+\tau-4 \tau^{2} / 3 \sigma>0$. This fulfills the condition that $\beta-\gamma+\tau-\tau^{2} / \sigma>0$. In that case the firm will indeed charge a price at $p_{[S]}^{*}=\beta-\gamma+\tau-\tau^{2} / \sigma$ to deter the network.

As with the previous propositions, we demonstrate the existence of parameter settings that qualify the above two numbered items to be subgameperfect equilibrium outcomes by providing for each of them a set of example parameters in Table 3.4.

\begin{tabular}{l|cc}
\hline & \multicolumn{2}{|c}{ Equilibrium outcomes } \\
Parameters & $\Pi_{[S]}^{*}$ & $\Pi_{[S / N]}^{*}$ \\
\hline$\beta$ & 14 & 12 \\
$\gamma$ & 13 & 11 \\
$\sigma$ & 12 & 9.5 \\
$\tau$ & 10 & 9 \\
\hline
\end{tabular}

Table 3.4: Examples of parameter values for Proposition 3.4.3 (low heterogeneity scenario) 
The relevant profit functions given these two sets of parameter values are as depicted in Figure 3.8. The two pictures represent the two sets of parameter values depicted in Table 3.4 respectively. Notice this time the discontinuity in profit levels across market structures.

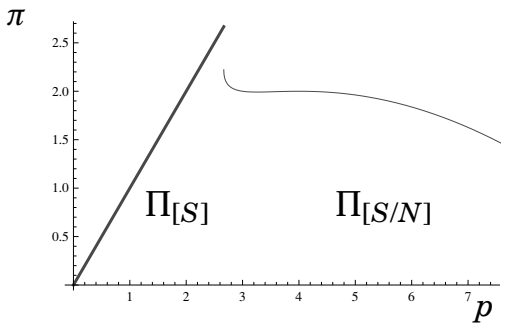

1. $[S]$

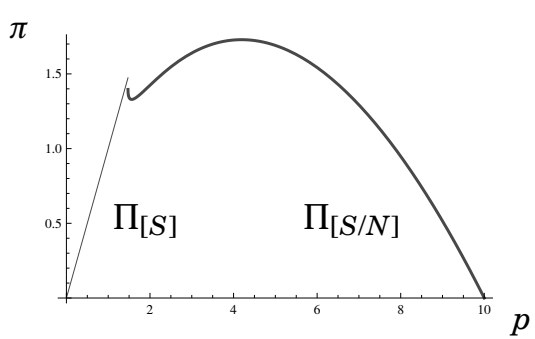

2. $[S / N]$

Figure 3.8: Profit functions and equilibrium outcomes (low heterogeneity scenario)

\subsection{Equilibrium market structures}

We summarize the analysis of the scenarios above by means of the following five corollaries, in which we show the optimal prices of the firm and the resulting market structures that are supported by a subgame-perfect Nash equilibrium.

Corollary 3.5.1. Partially-served local monopoly.

When $\tau$ is sufficiently large $(\tau>\gamma>\sigma$ ), the firm can act as a monopoly while ignoring the P2P network by charging a price $p^{*}=\beta / 2$ and making a profit of $\Pi_{[S / \phi / N]}^{*}=\beta^{2} / 4 \tau$. The outcome is a multi-platformed partially-served market $-[S / \varnothing / N]$.

This result is stemming from the high heterogeneity scenario. When taste heterogeneity $\tau$ is high enough, the consumers are sufficiently differentiated so that in equilibrium the firm can safely disregard the network at the other end of the market (those who prefer the digital form will therefore download) and charge its monopolistic price to its "own" consumers. The two platforms will, therefore, co-exist and not compete; and the market will be partially served. 
Corollary 3.5.1, in combination with Chapter 2, shows that $\tau>\gamma$ is a necessary condition for a multi-platformed partially-served market. One could interpret $\tau>\gamma$ as the situation that tastes differ so much across consumers that some consumers would not have the illegal version even if downloading it costs zero time and effort.

Corollary 3.5.2. Fully-served monopoly.

When $\tau$ is not too small, the firm can serve the entire market at the profitmaximizing price level $p^{*}=\beta-\gamma+\sigma-\tau$ and a profit level of $\Pi_{[S]}^{*}=\beta-\gamma+\sigma-\tau$, and the outcome is a single-platformed fully-served market $-[S]$.

This result is stemming from the high and medium heterogeneity cases. The firm's natural profit maximizing sales quantity is the entire market. This is mainly due to the fact that the basic utility of the physical product is so high that taste heterogeneity cost becomes insignificant in comparison. Although this is theoretically possible, in reality taste heterogeneity is probably high enough that in equilibrium not everyone will be naturally served by the monopoly. Therefore, we will not dig too much deeper in the analysis of this market outcome.

Corollary 3.5.3. Network deterrence.

When $\tau$ is very small $(\gamma>\sigma>\tau)$, the firm can serve the entire market and thereby deter the entry of the network by charging a price of $p^{*}=\beta-\gamma+\tau-$ $\tau^{2} / \sigma$, making a profit of $\Pi_{[S]}^{*}=\beta-\gamma+\tau-\tau^{2} / \sigma$, and the outcome is a singleplatformed fully-served market $-[S]$.

This result is stemming from the low heterogeneity scenario. Note that opposite to Corollary 3.5.1, where $\tau$ is the biggest, in Corollary 3.5.3, $\tau$ is the smallest. This proposition demonstrates that under certain parameter conditions, there is a possibility for the firm to deter the network from forming by an aggressive pricing strategy. Observe that this is only possible when taste heterogeneity is very small. Since consumers never fail to coordinate on forming a network (owing to Assumption 3.2.2), the only way for the firm to deter the network is to serve the whole market itself, even if, by doing so, it can no longer charge a monopoly price. Indeed, under these conditions, if the firm ignores the network and charges the normal monopoly price, the network would form and reach its maximum equilibrium size and 
cause some serious damage on the firm's sales. The firm is, therefore, better off deviating from the monopoly price and setting a network-deterring price.

\section{Corollary 3.5.4. Market sharing}

When $\tau$ is very large, the firm serves precisely all consumers who do not download by charging a price $p=\beta-\tau\left(\tau-\sqrt{\tau^{2}-4 \sigma(\tau-\gamma)}\right) / 2 \sigma$, and earns a profit of $\Pi_{[S / N]-\text { boundary }}=\left(\tau-\sqrt{4 \gamma \sigma-4 \sigma \tau+\tau^{2}}\right)\left(2 \beta \sigma-\tau^{2}+\tau \sqrt{4 \gamma \sigma-4 \sigma \tau+\tau^{2}}\right) / 4 \sigma^{2}$. The market outcome is a fully-served multi-platformed market without the two platforms competing with each other - $[S / N]-$ boundary.

This is a rather special market outcome, where the firm and the network together "share" the whole market without actually competing with each other. It relies on the fact that taste heterogeneity is sufficiently large such that the firm does not have to fight hard with the network for the indifferent consumer; but at the same time not so large such that the firm enjoys being a local monopoly as in Corollary 3.5.1. Note that the firm is actually pricing higher than its monopolistic price due to the presence of the network. For at the monopolistic price, the firm would face competition from the network and thereby unable to achieve monopolistic quantity. Thus, the firm sets the price at exactly the level so as to leave the network alone and served the entire residual demand of the market.

Corollary 3.5.5. Network accommodation.

Regardless of the value of $\tau$, there are parameter settings where the firm competes directly with the P2P network by charging a price $p^{*}=2(2 \tau-\sqrt{\delta})(\tau+$ $\sqrt{\delta}) / 9 \sigma$, making a profit of $\Pi_{[S / N]}^{*}=2(2 \tau-\sqrt{\delta})^{2}(\tau+\sqrt{\delta}) / 27 \sigma^{2}$, and the market outcome is a multi-platformed fully-served market $-[S / N]$.

This corollary captures all the rest of the parameter space which is not covered in Corollary 3.5.1, 3.5.2, 3.5.3 and 3.5.4. It is essentially the relation $\gamma>\sigma$ that makes the network readily available, and that makes network accommodation (co-existence) a prominent phenomenon.

The corollaries summarize the essential findings of this chapter and tells a rather compelling story. When consumers view the two versions as very different ( $\tau$ being large), the firm may enjoy the luxury of not having to deal with the network since its own clientele is by no means affected. For this to happen, the basic utility of the illegal version $\gamma$ should be relatively small 
and/or the generic cost factor of downloading $\sigma$ should be relatively high. In this case, aggressive price competition is not called for. When the taste differences are less pronounced, however, price competition with the network cannot be avoided. The firm has to accommodate the network, and they fight with each other for the marginal consumer. Together they serve the entire market. The situation changes even further when taste heterogeneity reaches a very low level. In that case, consumers no longer have high disutility for consuming the least preferred version, and the firm may have a chance to price aggressively in order to grab hold of the whole market and deter the network from forming.

Furthermore, $\delta=4 \tau^{2}-3 \sigma(\beta-\gamma+\tau)$ plays an important role in determining market outcomes. Recall that $\delta$ corresponds to the attractiveness of the network relative to the firm. For the firm to ignore the existence of the network, $\delta$ has to be relatively small. This requires, among other things, $\gamma$ to be relatively small and $\tau$ to be relatively large. It is consistent with what is required by Corollary 3.5.1. Alternatively, for the firm to be able to deter the entry of the network, $\delta$ has to be small as well. But Corollary 3.5.3 requires $\gamma$ to be large and $\tau$ to be small, which makes $\delta$ relatively large. This implies that $\sigma$ and $\beta$ have to be relatively large for $\delta$ to be small. Intuitively, if consumers do not care too much about the difference between versions, the firm can only deter the network if its product is much more superior in quality and the generic factor of downloading is relatively large. This may in reality prove hard to achieve.

\subsection{Comparative statics}

The previous section shows how taste heterogeneity $\tau$ can affect the possible equilibrium outcome in the market. It is essentially a comparative static analysis across equilibrium market structures. It may be interesting also to look at comparative statics of all the main parameters of the model in given equilibrium market structures. In Chapter 2 , the comparative statics of many market structures have been extensively studied, including the single-platformed as well as the multi-platformed fully-served markets. The main findings there are the following. The larger the generic cost factor of downloading is, the less likely a P2P network will form, and the higher the 
firm's profit will be. Once the market exhibits co-existence of the two platforms, the smaller the cost factor is, the higher the price set by the firm. Despite this pricing behavior, the firm's profit unambiguously declines as the cost factor decreases. In the partially-served monopolistic market with network-deterring pricing and the fully-served market with multi-platform co-existence, the total welfare decreases in the cost factor. For a comprehensive overview of this, we refer to Chapter 2.

Due to the different parameter settings in this chapter - smaller $\sigma$ and less restrictions on $\tau$ - two additional market outcomes can be supported by a subgame-perfect equilibrium. One is a naturally fully-served monopoly market $[S]$, and the other a multi-platformed partially-served local monopoly market $[S / \phi / N]$. We focus on the latter more interesting one and look at its comparative statics. Here the firm is ignoring the network all together. It charges a monopoly price $p^{*}$, serving a market the size of $s^{*}$, and making a profit of $\Pi^{*}$. The network lives at the other end of the market with a size of $n^{*}{ }^{6}$ We will also look at the effects on consumers' surplus and the total welfare, denoted by $C S^{*}$ and $W^{*}$, respectively.

Proposition 3.6.1. The signs of the first order partial derivatives of the equilibrium values with respect to the parameters in the case of the monopoly market are as shown in Table 3.5.

\begin{tabular}{lllll}
\hline & $\beta$ & $\gamma$ & $\tau$ & $\sigma$ \\
\hline$p^{*}$ & + & 0 & 0 & 0 \\
$s^{*}$ & + & 0 & - & 0 \\
$n^{*}$ & 0 & + & - & - \\
$\Pi^{*}$ & + & 0 & - & 0 \\
$C S^{*}$ & + & + & - & - \\
$W^{*}$ & + & + & - & - \\
\hline
\end{tabular}

Table 3.5: Multi-platformed partially-served (local monopoly) market.

Proof.

The comparative statics of all parameters on $p^{*}=\beta / 2, s^{*}=\beta / 2 \tau$, and $\Pi^{*}=$

\footnotetext{
${ }^{6}$ See Appendix 3.9.1 for the derivations.
} 
$\beta^{2} / 4 \tau$ are straight forward. Furthermore, the equilibrium network size $n^{*}=$ $1-\left(\tau-\sqrt{\tau^{2}-4 \sigma(\tau-\gamma)}\right) / 2 \sigma$ does not depend on $\beta$, and it is easy to see that it increases in $\gamma$. The first derivative of $n^{*}$ with respect to $\tau$ and $\sigma$ can be shown to be both negative.

The consumers' surplus can be divided into two components: the surplus for the buyers and the surplus for the downloaders, which we denote by $C S_{S}^{*}$ and $C S_{N}^{*}$, respectively. The expression for the former $C S_{S}^{*}=\beta^{2} / 8 \tau$. It is clear that the signs of its derivatives with respective to $\beta, \gamma, \tau$ and $\sigma$ are $+, 0,-$ and 0 . The expression of $C S_{N}^{*}$ is slightly more involved, but we know that it is the integral of the utility functions of the downloaders, which is $\gamma-\tau(1-x)-\sigma(1-n)^{2}$, with respect to $x$. It is clear that $\tau$ and $\sigma$ both have negative effects on each downloader's utility. Moreover, we know that they also have negative effects on the network size $n$. This shows that the total effects of $\tau$ and $\sigma$ must be negative on downloaders' surplus $C S_{N}^{*}$. Combined with the effects on the buyers' surplus $C S_{S}^{*}$, it can be seen that the effects of $\beta, \gamma, \tau$ and $\sigma$ on consumers' surplus $C S^{*}$ are,,++- and -, respectively. The effects on the total welfare are then simply the sum of the effects on consumers' surplus $C S^{*}$ and the firm's profit $\Pi^{*}$, which are unambiguous.

The intuition of the negative effect of the generic cost factor of downloading $\sigma$ on the equilibrium network size $n^{*}$ is self-evident: a higher cost factor makes the network less attractive and therefore resulting in a smaller equilibrium network. The cost factor does not have an effect on the surplus of buyers of the physical product, but does have a negative affect on that of the downloaders. This is reflected both by the direct multiplicative effect of downloading cost and the indirect negative effect of $\sigma$ on the equilibrium network size $n^{*}$. Since $\sigma$ does not affect the firm's profit, it does not inflict further harm to the society other than crippling the downloaders.

The negative welfare effects of taste heterogeneity $\tau$ are rather logical. Firstly, $\tau$ has a negative effect on the firm's profit $\Pi^{*}$, because for given basic utility $\beta$, a high $\tau$ leads to a lower sales quantity while the price remains constant. Thus, despite the fact that the firm has a monopoly position caused by taste heterogeneity, its profit suffers when $\tau$ increases. Next, the consumers' surplus of the buyers of the physical product is negatively affected by $\tau$, not 
because of the price, but because of the disutility of transportation cost for each consumer and the smaller equilibrium sales quantity $s^{*}$. The surplus from the downloaders is even more negatively affected by $\tau$, not only because $\tau$ represents the transportation cost deducted from the utility, but also because $\tau$ decreases the equilibrium network size $n^{*}$ which is in turn reflected in a higher downloading cost (a negative utility). Taste heterogeneity in this case is undoubtedly bad for the society as a whole.

\subsection{Discussion}

It might be helpful to combine the findings of this chapter and those of Chapter 2 into the same context, in order to have a complete picture of the matter at hand. The key issues here obviously are the legal anti-piracy enforcement (captured by the generic cost factor of downloading) and the difference in taste in the society with regard to legal and illegal copies (captured by taste heterogeneity), and the way the society is influenced by these factors, both the consumers and the firm.

When the legal enforcement level is high (high cost factor of downloading), networks may fail to form and the firm in a lot of cases may have the possibility to deter the network using aggressive pricing or to ignore the network all together. In those cases, the firm has little worries. However, when the enforcement level is low, as we have seen in this chapter, the firm is in much worse shape. Most of the network-deterring conditions will not be met and the firm has to accommodate the network and compete in price and sacrifice both on price and sales quantity, and thereby profit.

Only when taste heterogeneity is low, the firm may have a chance to deter the network but only if it is ready to cover the entire market with aggressive pricing. This will only work if the level of legal enforcement is reasonably high. Needless to say, the firm should ensure the superior quality of the physical product, like in our newspaper market example.

At the other end of the scale, where taste heterogeneity is so huge that the two plat-forms co-exist without competing for consumers leaving a section of market unserved is probably easier to conceive since most real-life markets are partially served.

Finally, it might be worth stressing that the total welfare in all of the 
possible market outcomes we have found in the model unambiguously benefits from low legal enforcement and low taste heterogeneity. The reason for the former is that low enforcement fosters a strong potential network, which either directly provides consumers' surplus by means of downloads or indirectly cuts deadweight loss by putting pressure on the price and thereby increasing sales quantity. The reason for the latter is simply the fact that taste heterogeneity can be considered as a sort of transportation cost that is subtracted from the consumers' surplus without going into anyone else's surplus.

To sum up all the analysis that have been done around this model, it is the probably realistic to consider in most of the markets for information goods today the firms are accommodating networks. Furthermore, the analysis has given us some insights on how the market outcomes can be different when some of the factors we observe today were to change. It also gives suggestions to the impact on the firm's profits and the total welfare, as a result of these possible changes.

\subsection{Concluding remarks}

This chapter adopts the model from Chapter 2 and changes the parameter settings to accommodate the cases with inherently strong P2P networks. In particular, we consider that there are always some "die-hard" P2P fans who contribute to the network's existence, and we allow the cases for relatively high level of consumer taste heterogeneity. As a result, the fight against a P2P network becomes much tougher as the consumers rarely fail to coordinate on forming a network. We find in total five equilibrium market structures, although under most conditions, the network will prevail and the firm can do nothing else than accommodating it and pricing against it to serve the entire market together. In this case, the firm's profit suffers and the total welfare benefits from the existence of P2P.

There may be some special conditions under which the firm still might have the upper hand. The firm can, for instance, find itself in the position to want to optimally serve the whole market, if the value of its product is significantly higher than that of the pirated version, and taste heterogeneity is low. Another example is when the subjective taste heterogeneity becomes 
very small, coupled with a relatively high value of the physical form comparing to the digital form, the firm might be able to charge a low enough price to deter the network from forming. One practical example could be the newspaper market. The value of a particular newspaper might drop drastically when it is one-day old. Therefore the value of an original newspaper may be considerably higher than a copied version. Thus if the newspaper is priced competitively enough, it might completely prevent anyone from having any incentives to share it on the P2P networks.

Another rather special market structure is market-sharing, which happens when taste heterogeneity is just so that the firm chooses to precisely serve the consumers who prefer not to download. In this situation the firm is actually pricing higher than its monopolistic price due to the presence of the network. For at the monopolistic price, the firm would face competition from the network and thereby unable to achieve the monopolistic quantity. Thus, the firm sets the price at exactly the level such that the consumer who is indifferent between downloading and not consuming will actually buy the physical form of the product from the firm. In other words, the firm lets the network form to its maximum size and serves the residual demand of the market.

The other end of spectrum is when taste heterogeneity is huge. In this case, the products are sufficiently differentiated and the firm and the network are co-existing as local monopolies without competition. Under this multi-platformed partially-served market structure, the quality of the two versions of the product positively affect the price, the sales quantity and the profit of the firm, as well as the consumers' surplus and hence welfare as a whole. Taste heterogeneity unambiguously hurts the sales, the profits and consumers' surplus and hence total welfare, although it has no effect on the price. It is clear that it is the large taste heterogeneity that gives rise to this monopolistic market structure in the first place, so it is not surprising that it hurts welfare, although it is interesting to observe that it also hurts the firm's profit. The generic cost factor of downloading $\sigma$ decreases total welfare because it hurts the downloading consumers but does not benefit the purchasing consumers or the firm's profit.

Finally, it is useful to stress that except for these special conditions mentioned above, the firm has no other option but to face the network and com- 
pete head-on with it in price. The resulting equilibrium market structure is the multi-platformed fully-served market where the firm and the network co-exist and compete for the marginal consumer. This is much studied in Chapter 2, and most important results in that chapter still hold here, except for the fact that the parameter space for this equilibrium market structure may be more pervasive in this chapter.

The pervasiveness of the co-existence market structure in this chapter may explain why, despite all the effort from the authorities and the publishing firms in the chasing and hunting of $\mathrm{P} 2 \mathrm{P}$ users, the results have not been hugely impressive, and internet piracy is still by and large an eminent phenomenon. Perhaps it is precisely those few, who believe in and stand by the principle of sharing and continue to provide the vital "critical mass" to the rest, that contribute to the prevalence of such file-sharing communities. And given the technology and the dispersion of the user base of such networks, it is less and less likely for anyone to technically eliminate such file sharing. It looks like the information good providers of the 21 st century really have their work cut out for them.

\subsection{Appendix}

\subsubsection{Solutions of the multi-platformed market structures}

\section{Fully-served market}

The consumer $z \in(0,1)$ that separates the consumers choosing for the firm from those choosing for the network, is herself indifferent between the two options. Moreover this consumer should weakly prefer the firm to the option not to acquire the product. So, for a second-stage equilibrium to generate the structure $[S / N]$, it should hold that, given the choices of everyone else,

$$
0 \leq U^{z}(p, S)=U^{z}(p, N) \quad \Longleftrightarrow \quad 0 \leq \beta-\tau z-p=\gamma-\tau(1-z)-\sigma(1-n(p))^{2},
$$

where $n(p)=1-z$, since the market is fully-served. Solving the inequality for $z$ gives us two solutions:

$$
z_{c . m .}(p)=\frac{\tau+\sqrt{\sigma(p+\gamma-\beta-\tau)+\tau^{2}}}{\sigma} \quad \text { and } \quad z_{m . n .}(p)=\frac{\tau-\sqrt{\sigma(p+\gamma-\beta-\tau)+\tau^{2}}}{\sigma},
$$


where we call the solution corresponding to the smaller network size $\left(z_{c . m .}\right)$ the critical-mass network, and the solution corresponding to the larger network size $\left(z_{m . n}\right.$. $)$ the maximum network. The solutions will only be real if $\sigma(p+\gamma-\beta-\tau)+\tau^{2} \geq 0$, which puts a condition on prices and will be discussed later in more detail. Moreover, $z \in(0,1)$ will be a necessary condition for the relevant parameters settings.

\section{Partially-served market}

The consumer $z^{S} \in(0,1)\left(z^{N} \in(0,1)\right)$ that separates the consumers choosing for the firm (network) from those choosing not to acquire any product, is herself indifferent between the two options. Moreover, this consumer prefers both the firm (network) and no acquisition to the option to acquire via the network (firm). We have a partially-served market if there is an interval of consumers that prefers no acquisition to acquisition, meaning that $z^{S}$ is less than $z^{N}$. So, for a second-stage equilibrium to generate the structure $[S / \phi / N]$, it should hold that, given the choices of everyone else,

$$
U^{z^{S}}(p, S)=U^{z^{S}}(p, \varnothing) \text { and } U^{z^{N}}(p, N)=U^{z^{N}}(p, \varnothing)
$$

and

$$
0 \leq z^{S}(p)<z^{N}(p) \leq 1 .
$$

The first two equations are equivalent to

$$
\beta-\tau z^{S}-p=0 \text { and } \gamma-\tau\left(1-z^{N}\right)-\sigma(1-n(p))^{2}=0 .
$$

where $n(p)=1-z^{N}$. Solving the first equation for $z^{S}$ and the latter for $z^{N}$ gives us one solution for $z^{S}$ :

$$
z^{S}(p)=\frac{\beta-p}{\tau},
$$

and two solutions for $z^{N}$ :

$$
z_{c . m .}^{N}=\frac{\tau+\sqrt{\tau^{2}+4 \sigma(\gamma-\tau)}}{2 \sigma} \quad \text { and } \quad z_{m . n .}^{N}=\frac{\tau-\sqrt{\tau^{2}+4 \sigma(\gamma-\tau)}}{2 \sigma} .
$$

Again, these solutions will only be real if $\tau^{2}+4 \sigma(\gamma-\tau) \geq 0$, which puts a condition on the relevant parameter settings and will be explained later in more detail. 


\subsubsection{Supports of Proposition 3.3.1-3.3.3}

\section{Proposition 3.3.1}

The following shows the feasible market structures for the high heterogeneity scenario $(\tau>\gamma>\sigma)$ and the price range for each market structure.

$[S]$ requires that all consumers prefer the physical version to the digital version, including the consumer located at $x=1$ whose choice constitutes the binding condition,

$$
\beta-\tau-p \geq \gamma-\sigma \Longleftrightarrow p \leq \beta-\gamma+\sigma-\tau
$$

Hence, this is the maximum price with which the firm can grab hold of the whole market. A price any higher than this, the market will turn into $[S / N]$, which we will investigate little later on. Note that for this to be an equilibrium market structure, $\beta-\gamma+\sigma-\tau \geq 0$ is a necessary condition.

A necessary condition for the market structure $[N]$ to exist is that the consumer located at $x=0$ must have positive utility from downloading when everyone else is downloading. This requires $\gamma-\tau(1-0)-\sigma(1-1)^{2}>0 \Longleftrightarrow$ $\gamma>\tau$, which is in conflict with the parameter setting in this scenario. We conclude, therefore, that in the high heterogeneity scenario $[N]$ does not exist.

$[\varnothing / N]$ though does exist in principle, and the condition for that is that the firm charges a price so high, i.e. when $p>\beta$, that no one buys the physical product from the firm. But this is not that interesting since in equilibrium $p>\beta$ will never be the case.

Next, under the market structure of $[S / N]$, there is a consumer located at $x=z$ who is indifferent from buying and downloading. The conditions that have to be fulfilled are the utilities of these choices are equal, positive, and the location of $z$ is within $(0,1)$. Formally,

$$
\begin{aligned}
\beta-\tau z-p & =\gamma-\tau(1-z)-\sigma z^{2} \\
0 & <z<1 \\
\beta-\tau z-p & \geq 0
\end{aligned}
$$

As explained in Appendix 3.9.1, solving Equation (3.1) yields two solutions for $z$ :

$$
z_{c . m .}(p)=\frac{\tau+\sqrt{\sigma(p+\gamma-\beta-\tau)+\tau^{2}}}{\sigma} \quad \text { and } \quad z_{m . n .}(p)=\frac{\tau-\sqrt{\sigma(p+\gamma-\beta-\tau)+\tau^{2}}}{\sigma},
$$


In order to guarantee the solution to be real, we need

$$
p \geq \beta-\gamma+\tau-\frac{\tau^{2}}{\sigma} .
$$

It can be easily seen that $z_{c . m .}>1$, as $\sqrt{\sigma(p+\gamma-\beta-\tau)+\tau^{2}} \geq 0$ and $\tau>\sigma$, and hence the critical mass equilibrium does not exist in this scenario.

Let us now look at the unique solution, the maximum network. Inequality (3.2) essentially has two components. The first component $0<z$ can be expressed as

$$
\begin{gathered}
\frac{\tau-\sqrt{\sigma(p+\gamma-\beta-\tau)+\tau^{2}}}{\sigma}>0 \Longleftrightarrow \tau-\sqrt{\sigma(p+\gamma-\beta-\tau)+\tau^{2}}>0 \\
\Longleftrightarrow \sigma(p+\gamma-\beta-\tau)<0 \Longleftrightarrow p<\beta-\gamma+\tau
\end{gathered}
$$

The second component $z<1$ can be expressed as

$$
\begin{gathered}
\frac{\tau-\sqrt{\sigma(p+\gamma-\beta-\tau)+\tau^{2}}}{\sigma}<1 \Longleftrightarrow \sqrt{\sigma(p+\gamma-\beta-\tau)+\tau^{2}}>\tau-\sigma \\
\Longleftrightarrow p+\gamma-\beta-\tau>-2 \tau+\sigma \Longleftrightarrow p>\beta-\gamma+\sigma-\tau
\end{gathered}
$$

Note that this coincides exactly the upper-bound price level of the market structure $[S]$. In other words, this is the price level where $[S]$ stops and $[S / N]$ starts. Finally, Inequality (3.3) requires

$$
\beta-\tau \cdot \frac{\tau-\sqrt{\sigma(p+\gamma-\beta-\tau)+\tau^{2}}}{\sigma}-p \geq 0 \Longleftrightarrow \sqrt{\sigma(p+\gamma-\beta-\tau)+\tau^{2}} \geq \tau-\frac{\beta-p}{\tau} \cdot \sigma .
$$

To further simplify this inequality, we need to examine two cases. The first case is when $\tau-\sigma(\beta-p) / \tau<0$, which implies that Inequality (3.3) would be trivially satisfied. It also means that $p<\beta-\tau^{2} / \sigma$. But for this to hold together with Inequality (3.4), it must be that $\gamma>\tau$, which is in conflict with the parameter settings of this scenario. It leaves us the second case where $p \geq \beta-\tau^{2} / \sigma$, which implies,

$$
\sigma(p+\gamma-\beta-\tau)+\tau^{2} \geq \tau^{2}+\frac{\sigma^{2}}{\tau^{2}}(\beta-p)^{2}-2 \sigma(\beta-p) .
$$

Solving this inequality for $p$ yields,

$$
\beta-\frac{\tau}{2 \sigma}\left(\tau+\sqrt{\tau^{2}-4 \sigma(\tau-\gamma)}\right) \leq p \leq \beta-\frac{\tau}{2 \sigma}\left(\tau-\sqrt{\tau^{2}-4 \sigma(\tau-\gamma)}\right) .
$$


The condition on the left-hand side of Inequality (3.5) is redundant because $\beta-\tau\left(\tau+\sqrt{\tau^{2}-4 \sigma(\tau-\gamma)}\right) / 2 \sigma \leq \beta-\gamma+\sigma-\tau$. The condition on the right-hand side of Inequality (3.5) is the upper bound of the price in $[S / N]$, as it is smaller than $\beta-\gamma+\tau .^{7}$ Naturally for this price to be real, the condition $\tau^{2}-4 \sigma(\tau-\gamma) \geq 0$ must hold.

Finally, when the market structure $[S / \varnothing / N]$ prevails, there will be two indifferent consumers: the one who is indifferent between buying the physical product from the firm and not consuming at all, and the other who is indifferent from downloading from $\mathrm{P} 2 \mathrm{P}$ and not consuming at all. Let the former be located at $x=z^{S}$, then it must be that his utility of buying the physical product is equal to not consuming which is equal to 0 .

$$
\beta-\tau z^{S}-p=0 .
$$

Solving it for $z^{S}$ yields $z^{S}=(\beta-p) / \tau$. Let the consumer who is indifferent from downloading and not consuming be located at $x=z^{N}$, then we have

$$
\gamma-\tau\left(1-z^{N}\right)-\sigma\left(z^{N}\right)^{2}=0 .
$$

Solving it for $z^{N}$, we obtain two solutions: $z_{m . n \text {. }}^{N}=\left(\tau-\sqrt{\tau^{2}-4 \sigma(\tau-\gamma)}\right) / 2 \sigma$ and $z_{c . m .}^{N}=\left(\tau+\sqrt{\tau^{2}-4 \sigma(\tau-\gamma)}\right) / 2 \sigma$, where, as before, $z_{m . n .}^{N}$. refers to the maximum network, and $z_{c . m}^{N}$. the critical mass network. Again it is easy to see that $z_{c . m .}^{N}>1$, i.e. the critical mass network solution does not exist. ${ }^{8}$ The unique solution is then $z_{m . n .}^{N}$, which is only positive if $\tau>\gamma>\sigma$. This corresponds precisely to the parameter settings of this scenario. Furthermore, for the market structure $[S / \varnothing / N]$ to exist, the following condition has to be fulfilled.

$$
0<z^{S}<z_{m . n .}^{N}<1
$$

The left part of the inequality, $z^{S}>0$, is simply $(\beta-p) / \tau>0 \Longleftrightarrow p<\beta$. The right part of the inequality, $z_{m . n .}^{N}<1$, always holds. ${ }^{9}$ Now the middle part of

${ }^{7}$ It is easy to verify that $\beta-\tau\left(\tau-\sqrt{\tau^{2}-4 \sigma(\tau-\gamma)}\right) / 2 \sigma<\beta<\beta-\gamma+\tau$.

${ }^{8}$ It requires $\left(\tau+\sqrt{\tau^{2}-4 \sigma(\tau-\gamma)}\right) / 2 \sigma>1 \Longleftrightarrow \sqrt{\tau^{2}-4 \sigma(\tau-\gamma)}>2 \sigma-\tau$. If $2 \sigma<\tau$, this is trivially true; and if $2 \sigma \geq \tau$, then it requires that $\tau^{2}-4 \sigma(\tau-\gamma)>\tau^{2}-4 \tau \sigma+4 \sigma^{2} \Longleftrightarrow \gamma>\sigma$, which is true.

${ }^{9}$ It requires $\left(\tau-\sqrt{\tau^{2}-4 \sigma(\tau-\gamma)}\right) / 2 \sigma<1 \Longleftrightarrow \sqrt{\tau^{2}-4 \sigma(\tau-\gamma)}>\tau-2 \sigma$. If $\tau<2 \sigma$, this is trivially true; and if $\tau \geq 2 \sigma$, then it requires that $\tau^{2}-4 \sigma(\tau-\gamma)>\tau^{2}-4 \tau \sigma+4 \sigma^{2} \Longleftrightarrow \gamma>\sigma$, which is true. 
Inequality (3.6) is the only thing that puts a restriction on the price range of $[S / \varnothing / N]$. It requires $\left(\tau-\sqrt{\tau^{2}+4 \sigma(\gamma-\tau)}\right) / 2 \sigma>(\beta-p) / \tau \Longleftrightarrow \sqrt{\tau^{2}+4 \sigma(\gamma-\tau)}<$ $\tau-2 \sigma(\beta-p) / \tau \Longleftrightarrow p>\beta-\tau\left(\tau-\sqrt{\tau^{2}-4 \sigma(\tau-\gamma)}\right) / 2 \sigma$.

\section{Proposition 3.3.2}

The following shows the feasible market structures for the medium heterogeneity scenario $(\gamma>\tau>\sigma)$ and the price range for each market structure.

The market structure $[S]$ is still possible, and as conditions are the same as in the high heterogeneity scenario, so is the price range $p \leq \beta-\gamma+\sigma-\tau$. Any price higher than that, we will be in the market structure of $[S / N]$.

For the market structure $[S / N]$, we will again have a unique solution; i.e. the maximum network equilibrium $z_{m . n .}=\left(\tau-\sqrt{\sigma(p+\gamma-\beta-\tau)+\tau^{2}}\right) / \sigma$. This is because $z_{c . m}>1$, hence the critical mass network equilibrium does not exist. Thus, for $[S / N]$ to exist, the following conditions have to be fulfilled.

$$
\begin{gathered}
0<z_{m . n .}<1 \\
\beta-\tau \cdot z_{m . n .}-p \geq 0
\end{gathered}
$$

The left-hand side of Inequality (3.7) requires

$$
\begin{gathered}
\frac{\tau-\sqrt{\sigma(p+\gamma-\beta-\tau)+\tau^{2}}}{\sigma}>0 \Longleftrightarrow \sqrt{\sigma(p+\gamma-\beta-\tau)+\tau^{2}}<\tau \\
\Longleftrightarrow \sigma(p+\gamma-\beta-\tau)<0 \Longleftrightarrow p<\beta-\gamma+\tau .
\end{gathered}
$$

The right-hand side of Inequality (3.7) requires

$$
\frac{\tau-\sqrt{\sigma(p+\gamma-\beta-\tau)+\tau^{2}}}{\sigma}<1 \Longleftrightarrow \sqrt{\sigma(p+\gamma-\beta-\tau)+\tau^{2}}>\tau-\sigma \Longleftrightarrow p>\beta-\gamma-\tau+\sigma
$$

We will now show that Inequality (3.8) does not further restrict the price. Inequality (3.8) is equivalent to $\sqrt{\sigma(p+\gamma-\beta-\tau)+\tau^{2}} \geq \tau-\sigma(\beta-p) / \tau$. In case the right-hand side of this inequality is negative, i.e. in case $p<\beta-\tau^{2} / \sigma$, the inequality is trivially satisfied. Otherwise, $p \geq \beta-\tau^{2} / \sigma$, then Inequality (3.8) implies $\sigma(\beta-p)^{2} / \tau^{2}-(\beta-p)-(\gamma-\tau) \leq 0$, which is equivalent to $\beta-\tau(\tau+$ $\left.\sqrt{\tau^{2}+4 \sigma(\gamma-\tau)}\right) / 2 \sigma \leq p \leq \beta-\tau\left(\tau-\sqrt{\tau^{2}+4 \sigma(\gamma-\tau)}\right) / 2 \sigma$. Because $\gamma>\tau$, the lower bound on $p$ is less than $\beta-\tau^{2} / \sigma$ and therefore satisfied since we are considering the case $p \geq \beta-\tau^{2} / \sigma$. The upper bound is larger than $\beta$. Hence Inequality (3.8) does not further restrict the price in either direction. 
Now we show that the multi-platformed, partially-served market [S/ $\varnothing / N]$ can not exist in the medium heterogeneity scenario. Consider the consumer who is indifferent from downloading and not consuming. Similar to the previous scenario, we solve for the condition of indifference and obtain two solutions: $z_{m . n .}^{N}=\left(\tau-\sqrt{\tau^{2}+4 \sigma(\gamma-\tau)}\right) / 2 \sigma$ and $z_{c . m .}^{N}=\left(\tau+\sqrt{\tau^{2}+4 \sigma(\gamma-\tau)}\right) / 2 \sigma$. It is easy to see that, given $\gamma>\tau, z_{m . n .}^{N}<0$ and $z_{c . m .}^{N}>1$. This means that neither of these two solution exists.

Finally, opposite to the case in the high heterogeneity scenario, the market structure $[\varnothing / N]$ can not exist but $[N]$ can, because $\gamma>\tau$. The condition for this market structure to prevail is that the consumer located at $x=0$ prefers downloading from $\mathrm{P} 2 \mathrm{P}$ to buying from the firm. This implies $\gamma-\tau>\beta-p \Longleftrightarrow p>\beta-\gamma+\tau$.

\section{Proposition 3.3.3}

The following shows the feasible market structures for the low heterogeneity scenario $(\gamma>\sigma>\tau)$ and the price range for each market structure.

The market structure $[S]$ is once again similar as two previous scenarios, and the price range is $p \leq \beta-\gamma+\sigma-\tau$. At a higher price, the market structure will be $[S / N]$. Unlike the previous two scenarios, the market structure $[S / N]$ in this scenario is slightly more involved. In particular, both the critical mass network and the maximum network solutions exist, which causes multiple equilibria in the second stage.

We first look at the maximum network solution $z_{m . n .}$. The requirements for existence are as usual:

$$
\begin{gathered}
0 \leq z_{m . n .}<1 \\
\beta-\tau \cdot z_{m . n .}-p \geq 0
\end{gathered}
$$

The left-hand side of Inequality (3.9) requires, as in the medium heterogeneity scenario, $p \leq \beta-\gamma+\tau$. This is the upper-bound price for the market structure $[S / N]_{m . n .}$. The right-hand side, however, leads to a different condition due to the fact that $\tau<\sigma$.

$$
\frac{\tau-\sqrt{\sigma(p+\gamma-\beta-\tau)+\tau^{2}}}{\sigma}<1 \Longleftrightarrow \sqrt{\sigma(p+\gamma-\beta-\tau)+\tau^{2}}>\tau-\sigma .
$$

Since $\sigma>\tau$ in this scenario, the condition is satisfied whenever the square 
root exists, which is when $p>\beta-\gamma+\tau-\tau^{2} / \sigma$. Similar to the medium heterogeneity scenario, Inequality (3.10) does not further restrict the prices.

The lower-bound price of $[S / N]_{m . n}$. is therefore $\beta-\gamma+\tau-\tau^{2} / \sigma$, which is below the upper-bound price of $[S], \beta-\gamma+\sigma-\tau$. This can be shown by supposing if the opposite were true, namely $\beta-\gamma+\tau-\tau^{2} / \sigma>\beta-\gamma+\sigma-\tau$. After rearranging, we get $2 \tau-\tau^{2} / \sigma-\sigma>0$. Multiplying both sides by $\sigma$ and rearranging yields $(\tau-\sigma)^{2}<0$, which is clearly impossible. Therefore the price in the market structure $[S / N]_{m . n}$. satisfies $\beta-\gamma+\tau-\tau^{2} / \sigma<p<\beta-\gamma+\tau$.

Furthermore, we need to investigate the critical mass solution $z_{c . m}$. in this scenario. The requirements for existence are similar to those of $z_{m . n}$, namely,

$$
\begin{gathered}
0 \leq z_{c . m .}<1 \\
\beta-\tau \cdot z_{c . m .}-p \geq 0
\end{gathered}
$$

The left-hand side of Inequality (3.11) is trivially satisfied. The right-hand side can be simplified to $p<\beta-\gamma+\sigma-\tau$. Inequality (3.12) is equivalent to

$$
\sqrt{\sigma(p+\gamma-\beta-\tau)+\tau^{2}} \leq \frac{\sigma}{\tau}(\beta-p)-\tau .
$$

This condition holds only if the right-hand side of this inequality is nonnegative, that is $p \leq \beta-\tau^{2} / \sigma$, and

$$
\frac{\sigma}{\tau^{2}}(\beta-p)^{2}-(\beta-p)-(\gamma-\tau) \geq 0,
$$

where the latter inequality is equivalent to

$$
p \leq \beta-\frac{\tau}{2 \sigma}\left(\tau+\sqrt{\tau^{2}+4 \sigma(\gamma-\tau)}\right) \quad \text { or } \quad p \geq \beta-\frac{\tau}{2 \sigma}\left(\tau-\sqrt{\tau^{2}+\sigma(\gamma-\tau)}\right) .
$$

Since $\gamma>\tau$, the right-hand side of the second inequality exceeds $\beta$ and hence cannot be satisfied-leaving the first inequality to be satisfied. Moreover, the right-hand side of the first inequality is less than $\beta-\tau^{2} / \sigma$, hence Inequality (3.12) is satisfied if and only if

$$
p \leq \beta-\frac{\tau}{2 \sigma}\left(\tau+\sqrt{\tau^{2}+4 \sigma(\gamma-\tau)}\right) .
$$

Now we need to see if this poses a further restriction on the upper-bound price. Since $\gamma>\tau$, we know that $\sqrt{\tau^{2}+4 \sigma(\gamma-\tau)}>\tau$. Because $\tau<\sigma$, it is then easy to see that $\beta-\tau\left(\tau+\sqrt{\tau^{2}+4 \sigma(\gamma-\tau)}\right) / 2 \sigma>\beta-\tau$. But because $\gamma>\sigma$, 
we also know that $\beta-\gamma+\sigma-\tau<\beta-\tau$. This implies that Inequality (3.12) does not pose further restrictions on the upper-bound price. Therefore, the price boundaries for the market structure $[S / N]_{c . m}$. are $\beta-\gamma+\tau-\tau^{2} / \sigma<p<$ $\beta-\gamma-\tau+\sigma$.

Finally, the market structure $[N]$ is the same as in the medium heterogeneity scenario, namely, $p>\beta-\gamma+\tau$. 



\section{Chapter 4}

\section{A dynamic point of view}

\subsection{Introduction}

The previous chapters are static in nature. ${ }^{1}$ This implies that in the presence of demand side externalities there may be multiple equilibria, which ultimately leads to an equilibrium selection of some sort. Consequently, coordination of the network formation is often assumed. The authors of these models always focus on the maximally achievable network size. These models, therefore, lack insights on how such networks actually form, step by step, from zero to a steady-state network size; how the firm prices strategically to compete with the network every step of the way; and how society is affected during the process.

Only a dynamic model can bring such insights, which is what this chapter is designed to achieve. Dynamic stochastic models with network externalities have been recently studied in the IO literature, mainly using numerical methods. Examples are (Markovich 2008) and (Markovich and Moenius 2009) which study the dynamics caused by the iterations between hardware and software; (Jenkins, Liu, Matzkin, and McFadden 2004) studies a stylized version of the browser war between Netscape and Microsoft, where the entrant may have "grabbed" market shares from the incumbent and thereby tipping the market; (Arie and Grieco 2009) investigate the effect of switching costs on market dominance and equilibrium prices; and (Chen,

\footnotetext{
${ }^{1}$ This chapter is an adapted version of the paper (Herings, Peeters, and Yang 2009).
} 
Doraszelski, and Harrington 2009) studies competing firms' incentives to make their products compatible and the possible effects that may prevent market dominance. A framework for numerically analyzing dynamic interactions in imperfectly competitive industries is proposed by (Doraszelski and Pakes 2007), which provides an excellent summary of the main approach for models of this kind.

In this chapter, we use a dynamic model to solve for the optimal pricing strategy of a firm that releases music CDs and sells them in the store while being exposed to a competing P2P file-sharing network on the internet. The reason why we choose the music industry is two-fold. Firstly, the music industry is allegedly one of the most severely damaged industries by online piracy - at least it has made the greatest amount of protest against $\mathrm{P} 2 \mathrm{P}$ file-sharing. It is therefore a good representative as the information content provider in the context of this thesis. Secondly, numerous empirical studies over the years have tried to explain the relationship between the decline in music sales and the rise of $\mathrm{P} 2 \mathrm{P}$ networks, thus providing us useful insights for calibrating the numerically computed dynamic model in this chapter. (Blackburn 2004), (Liebowitz 2004), (Rob and Waldfogel 2006), and (Zentner 2006) find that downloading (via P2P file-sharing networks) are at least partially responsible for the recent decrease in CD sales. (Liebowitz 2006) concludes that file-sharing has clearly brought significant harm to the recording industry. In contrast, (Oberholzer-Gee and Strumpf 2007) and (Peitz and Waelbroeck 2004) find little robust evidence that P2P has caused the decrease in CD sales for the recent years.

In the dynamic model of this chapter, there is a firm who sets the price of its CDs every period and a continuum of consumers who decide whether to purchase the CD from the store, download the music from P2P, or not acquire the music at all. The timing involves discrete periods with an infinite time horizon. The firm is forward looking and strives to maximize the present value of all future profits by choosing a state-dependent pricing policy; the state being the market shares of the store, the P2P network, and the unserved market. The consumers make one of the three choices based on the price and the state in order to maximize their utility. Consumers are ex ante identical, but receive random utility shocks prior to their purchasing decision every period anew. Switching costs are imposed on the consumers 
who switch to a product, from previously consuming the other product or not consuming at all. The firm's optimal pricing policy is derived numerically and provides insight in the market share dynamics. The parameters of the model have been calibrated in such a way that the model outcomes (such as prices and sales quantities) match the real-world data in the years 1999 and 2003.

An interesting point of this approach is that we can study how networks develop in a dynamic process without having to make any assumptions on consumer coordination as in the conventional static models of network economics. We can also observe exactly how the firm sets its price conditioned on its market share and that of the network. Sometimes it sets the price very low in order to win vital market shares to fight against the P2P network, while other times accommodates the network by setting a high price to reap the profit from its own installed customer base.

We could also use the model to predict future sales quantities and to compute the changes in consumers' surplus and total welfare. For instance, it predicts the sales quantity of the year 2008 rather accurately to be around 374.3 million copies, while the real figure is 384.7 million. It also predicts that the CD sales will have dropped to around 231.2 million copies per year around the year 2020, when according to the model estimations the long-run steady state is approximately reached. More importantly, this model is able to estimate the welfare gain over the years brought by the P2P file-sharing networks. In the year 2003, 4 years after P2P was introduced, while the music industry was suffering from a $\$ 1.6$ billion forgone profit per year, the total welfare was up by $\$ 13.1$ billion per year. By the year 2008 , the industry profit has dropped by a further $\$ 1.4$ billion, and welfare soared by a further $\$ 12.4$ billion per year, making it $\$ 25.6$ billion more than in 1999 . In the long-run steady state (around 2020), the industry profit will have dropped to a mere $\$ 865.5$ million per year, and total welfare are set to have improved since 1999 by a whopping $\$ 29.7$ billion per year.

In the comparative static analysis of the model, one of our most important findings is that total welfare is negatively related to the generic cost factor of downloading. This result coincides with the findings from Chapter 2, 3 and papers such as (Rob and Waldfogel 2006), in the sense that the existence of $\mathrm{P} 2 \mathrm{P}$ actually enhances total welfare. This implies that by 
making file-sharing more difficult for the consumers, the government is effectively curbing the society from enjoying a high welfare level.

The remainder of this chapter is organized as follows. In Section 4.2 the dynamic model is described in detail. Next, Section 4.3 explains how the numerical computations are conducted. In Section 4.4 the model is calibrated and the parameter values for the benchmark scenarios are set. The model outcomes and predictions are then presented in Section 4.5, and comparative statics are analyzed in Section 4.6. Section 4.8 concludes.

\subsection{The model}

Each period, music albums are being offered on CDs by the firm and online via P2P networks. A continuum of consumers decides whether to buy the album at the store $(S)$, to download it via P2P networks $(N)$, or not to acquire it at all $(E)$. A consumer's decision in one period determines her type in the subsequent period. That is, at each period, depending on the decision in the previous period, a consumer is of one of the three possible types: $\theta_{S}$ (store), $\theta_{N}$ (network), or $\theta_{E}$ (empty). The state at a certain period is defined as the distribution of consumers over types. Setting the total mass of consumers to 1 , the state space is given by

$$
\Omega=\left\{(s, n, e) \in \mathbb{R}_{+}^{3} \mid s+n+e=1\right\},
$$

where $s, n$, and $e$ represent the share of consumers of type $\theta_{S}, \theta_{N}$, and $\theta_{E}$, respectively. A typical state in $\Omega$ is denoted by $\omega$.

Every period, given the current state $\omega$, the firm sets a price for its CD in the store $p(\omega){ }^{2}$ This generates an immediate profit of

$$
\pi(\omega, p(\omega))=(p(\omega)-\mu) \cdot s(\omega, p(\omega))
$$

where $\mu$ represents the cost of producing one unit and $s(\omega, p(\omega))$ represents the resulting sales quantity, which equals the number of consumers choosing to buy the album in store given the current state $\omega$ and price $p(\omega)$. Hence,

\footnotetext{
${ }^{2}$ We assume that the firm exhibits stationary pricing behavior, which means that the price depends only on the state. It is well known that our framework possesses an optimal pricing policy in stationary strategies.
} 
in the next period the process will be in a state with $s(\omega, p(\omega))$ consumers of type $\theta_{S}$. When designing an optimal pricing scheme, the firm realizes that the price chosen in the current state does not only affect the immediate profit, but also the state transition and thus potential profits in the future. We assume the firm to be rational and farsighted. That is, in any period, it sets the price as to maximize the present value of the stream of profits discounted by a factor of $\delta$ each period.

Given the current market state $\omega$ and the firm's price at this state $p(\omega)$, the state transition is completely specified by the consumers' decisions. We assume consumers to maximize their utility in the current state. In her decision, a consumer takes into account the price at the store, the expected downloading costs, and possible switching costs $\tau$. The purpose of the switching costs $\tau$ in the model is to promote consumer loyalties to the platforms, reflecting a possible lock-in effect. In our model the expected downloading cost is negatively related to the network size and the switching cost is incurred only when a consumer acquires the product while changing type. Hence, a consumer's decision is determined by her type, the CD price at the store and the expected network size. Regarding the latter, we assume that consumers use last period's network size as a prediction for the network size in the current period. Formally, in state $\omega=(s, n, e)$ a consumer of type $\theta_{S}, \theta_{N}$, and $\theta_{E}$ maximizes her utility:

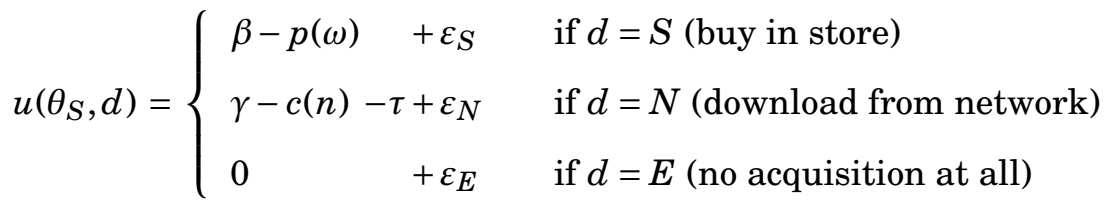

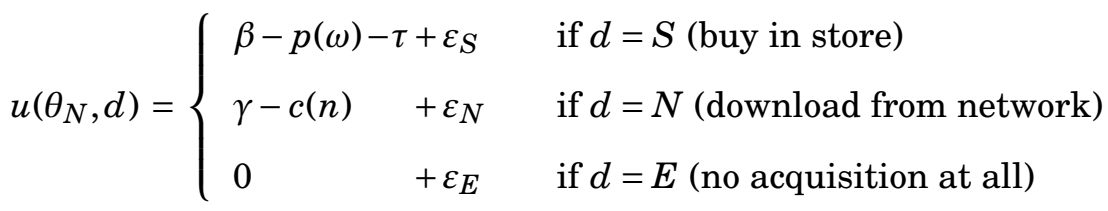

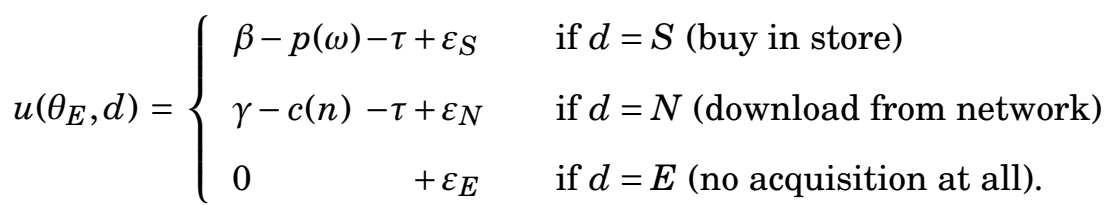

Here, $\beta>0$ and $\gamma>0$ represent the basic utility of the physical and the digital form respectively. The costs of downloading when the resulting network 
is of size $n \in[0,1]$ are represented by $c(n)$. A natural shape of this cost function is for it to be decreasing in the network size at a diminishing rate. This is due to the fact that the more users are sharing this file, the easier it is to acquire it from the P2P network. ${ }^{3}$ In our model we implement the following convex decreasing cost function:

$$
c(n)=\frac{\sigma}{\rho+n}, \quad n \in[0,1] .
$$

Here $\sigma>0$ represents the generic cost factor of downloading, incorporating a collection of factors that may affect downloading costs, for instance, the degree of legal enforcement of intellectual property rights. The parameter $\rho>0$ influences the curvature of the cost function. The smaller $\rho$ is, the steeper $c(n)$ is around the region where $n$ is close to 0 . Note that $\rho$ and $\sigma$ are identical for all consumers and are independent of the network size.

Finally, the terms $\varepsilon_{S}, \varepsilon_{N}$, and $\varepsilon_{E}$ are random terms leading to heterogeneous consumer behavior. We assume that these terms are independently drawn according to a Gumbel extreme value distribution with location parameter 0 and scale parameter $\lambda \geq 0$ for each consumer and each of the three alternative choices every period anew. The scale parameter $\lambda$ is inversely related to the level of heterogeneity among consumers' preferences.

Given the current state $\omega$ and the store's price at this state $p(\omega)$, the probability that a consumer with type $\theta$ is of type $\theta_{d}$ next period is then given by:

$$
q\left(\theta_{d} \mid \theta\right)[\omega, p(\omega)]=\frac{\exp (\lambda u(\theta, d))}{\exp (\lambda u(\theta, S))+\exp (\lambda u(\theta, N))+\exp (\lambda u(\theta, E))},
$$

for $\theta \in\left\{\theta_{S}, \theta_{N}, \theta_{E}\right\}$ and $d \in\{S, N, E\}$. Hence, from state $\omega=(s, n, e)$ with price $p(\omega)$, the process resumes in state $\omega^{\prime}=\left(s^{\prime}, n^{\prime}, e^{\prime}\right)$ next period, where

$$
\begin{aligned}
& s^{\prime}=s \cdot q\left(\theta_{S} \mid \theta_{S}\right)+n \cdot q\left(\theta_{S} \mid \theta_{N}\right)+e \cdot q\left(\theta_{S} \mid \theta_{E}\right), \\
& n^{\prime}=s \cdot q\left(\theta_{N} \mid \theta_{S}\right)+n \cdot q\left(\theta_{N} \mid \theta_{N}\right)+e \cdot q\left(\theta_{N} \mid \theta_{E}\right), \\
& e^{\prime}=s \cdot q\left(\theta_{E} \mid \theta_{S}\right)+n \cdot q\left(\theta_{E} \mid \theta_{N}\right)+e \cdot q\left(\theta_{E} \mid \theta_{E}\right) .
\end{aligned}
$$

We denote this process of state transitions by $Q: \Omega \times \mathbb{R}_{+} \rightarrow \Omega$.

\footnotetext{
${ }^{3}$ Notice that unlike local area networks, contemporary P2P networks are less prone to network congestions.
} 
The firm maximizes the present value of all future profits by implementing the price policy $p: \Omega \rightarrow \mathbb{R}_{+}$, that for all $\omega \in \Omega$ maximizes the value of

$$
V(\omega, p)=\pi(\omega, p(\omega))+\delta \cdot V(Q(\omega, p(w)), p),
$$

the Bellman equation for the firm's profit maximization problem.

The firm's search for the optimal pricing policy constitutes a Markov decision problem. To solve this problem, we turn to numerical methods. More precise, we discretize the state space and apply a policy iteration algorithm to find the optimal pricing strategy. Details on the numerical method are further explained in Section 4.3. The disretized version of our model is guaranteed to possess a stationary optimal pricing policy. The process of state transitions that is induced by this pricing policy provides insight in the implied market share dynamics. In our simulations presented in later sections, the state transition process leads to a unique invariant distribution. The set of states with positive probabilities in the invariant distribution is called the absorbing set. We refer to it as the long-run steady state or just the steady state in the non-technical parts of the chapter.

\subsection{Numerical method}

In this section, we explain how the model described in Section 4.2 is solved numerically. Firstly, we discretize the state space and adapt the firm's problem accordingly. Next, we apply the policy iteration algorithm ${ }^{4}$ on the discretized state space. Finally, we apply a bracketing algorithm ${ }^{5}$ to determine improvements in each state of the price policy.

\subsubsection{Discretization}

Given a natural number $k \geq 1$, we define the discretized state space by

$$
\widehat{\Omega}=\left\{(\widehat{s}, \widehat{n}, \widehat{e}) \in K^{3} \mid \widehat{s}+\widehat{n}+\widehat{e}=1\right\},
$$

where $K=\{0,1 / k, 2 / k, \ldots, 1\}$. Figure 4.1 illustrates graphically (for $k=4$ ) the state space and the discretized state space. The large triangle represents

\footnotetext{
${ }^{4}$ See (Howard 1960); (Blackwell 1962); see also (Judd 1998) p. 416.

${ }^{5}$ Cf. (Judd 1998).
} 
the state space. The corner points of this triangle represent the extreme states with full consumer mass at either Store, Network, or Empty. The interior points refer to states where the full mass of consumers is divided over the three platforms, the actual division being proportional to the proximity to the corner points. The triangulation of the triangle represents the discretized state space. The vertices are precisely the states in $\widehat{\Omega}$.

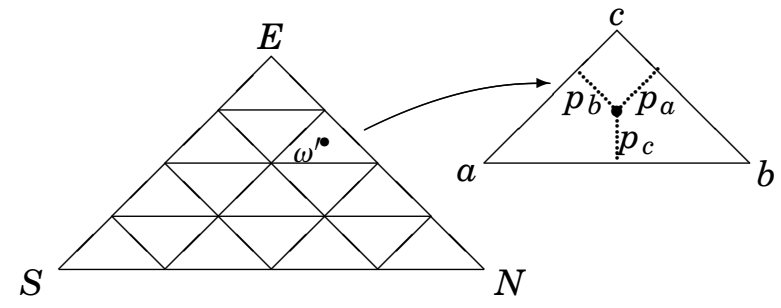

$$
\begin{aligned}
& a=\left(\left\lceil s^{\prime}\right\rceil,\left\lfloor n^{\prime}\right\rfloor,\left\lfloor e^{\prime}\right\rfloor\right) \\
& b=\left(\left\lfloor s^{\prime}\right\rfloor,\left\lceil n^{\prime}\right\rceil,\left\lfloor e^{\prime}\right\rfloor\right) \\
& c=\left(\left\lfloor s^{\prime}\right\rfloor,\left\lfloor n^{\prime}\right\rfloor,\left\lceil e^{\prime}\right\rceil\right) \\
& p_{a}=k \cdot\left(s^{\prime}-\left\lfloor s^{\prime}\right\rfloor\right) \\
& p_{b}=k \cdot\left(n^{\prime}-\left\lfloor n^{\prime}\right\rfloor\right) \\
& p_{c}=k \cdot\left(e^{\prime}-\left\lfloor e^{\prime}\right\rfloor\right)
\end{aligned}
$$

Figure 4.1: An illustration of the discretized state space and the discretized transition mapping.

Next, we define a transition mapping $\widehat{Q}: \widehat{\Omega} \times \mathbb{R}_{+} \rightarrow \Delta(\widehat{\Omega})$ on this discretized state space. Given current state $\widehat{\omega} \in \widehat{\Omega}$ and price policy $p: \widehat{\Omega} \rightarrow$ $\mathbb{R}_{+}$the process resumes in state $\omega^{\prime}=Q(\widehat{\omega}, p(\widehat{\omega}))$. Typically the new state $\omega^{\prime}=\left(s^{\prime}, n^{\prime}, e^{\prime}\right)$ is not an element of $\widehat{\Omega}$. In such a case we allocate probabilities to the nearest states in $\widehat{\Omega}$ with probabilities being proportional to the proximity to these states.

In order to provide a precise formulation of the discretized transition mapping it is convenient to define for a state $\omega^{\prime} \in \Omega$ the sets:

$$
\Delta\left(\omega^{\prime}\right)=\operatorname{ch}\left\{\left(\left\lceil s^{\prime}\right\rceil,\left\lfloor n^{\prime}\right\rfloor,\left\lfloor e^{\prime}\right\rfloor\right),\left(\left\lfloor s^{\prime}\right\rfloor,\left\lceil n^{\prime}\right\rceil,\left\lfloor e^{\prime}\right\rfloor\right),\left(\left\lfloor s^{\prime}\right\rfloor,\left\lfloor n^{\prime}\right\rfloor,\left\lceil e^{\prime}\right\rceil\right)\right\} \cap \Omega
$$

and

$$
\nabla\left(\omega^{\prime}\right)=\operatorname{ch}\left\{\left(\left\lfloor s^{\prime}\right\rfloor,\left\lceil n^{\prime}\right\rceil,\left\lceil e^{\prime}\right\rceil\right),\left(\left\lceil s^{\prime}\right\rceil,\left\lfloor n^{\prime}\right\rfloor,\left\lceil e^{\prime}\right\rceil\right),\left(\left\lceil s^{\prime}\right\rceil,\left\lceil n^{\prime}\right\rceil,\left\lfloor e^{\prime}\right\rfloor\right)\right\} \cap \Omega,
$$

where $\lfloor x\rfloor(\lceil x\rceil)$ refers to the nearest element in $K$ less (larger) than or equal to $x$. Depending on the location of $\omega^{\prime}$, four possible situations can occur. If $\omega^{\prime}$ is a vertex of the triangulated state space, then $\Delta\left(\omega^{\prime}\right)=\nabla\left(\omega^{\prime}\right)=\omega^{\prime}$. If $\omega^{\prime}$ is on an edge, then $\Delta\left(\omega^{\prime}\right)=\nabla\left(\omega^{\prime}\right)$ describes this edge. In case $\omega^{\prime}$ is in the interior of one of the subtriangles, $\omega^{\prime}$ is an element of either $\Delta\left(\omega^{\prime}\right)$ or $\nabla\left(\omega^{\prime}\right)$. When $\omega^{\prime}$ is located in an upward pointed triangle, $\omega^{\prime} \in \Delta\left(\omega^{\prime}\right)$ and $\nabla\left(\omega^{\prime}\right)=\varnothing$. Vice 
versa, when $\omega^{\prime}$ is located in an downward pointed triangle, $\omega^{\prime} \in \nabla\left(\omega^{\prime}\right)$ and $\Delta\left(\omega^{\prime}\right)=\varnothing$.

Contingent on $\omega^{\prime}$ being in $\Delta\left(\omega^{\prime}\right)$ or $\nabla\left(\omega^{\prime}\right)$, we specify the discretized transition probabilities. If $\omega^{\prime}=Q(\widehat{\omega}, p(\widehat{\omega}))$ is an element of $\Delta\left(\omega^{\prime}\right)$, then

$$
\widehat{Q}\left(\widehat{\omega}^{\prime} \mid \widehat{\omega}, p(\widehat{\omega})\right)= \begin{cases}k \cdot\left(s^{\prime}-\left\lfloor s^{\prime}\right\rfloor\right) & \text { if } \widehat{\omega}^{\prime}=\left(\left\lceil s^{\prime}\right\rceil,\left\lfloor n^{\prime}\right\rfloor,\left\lfloor e^{\prime}\right\rfloor\right) \\ k \cdot\left(n^{\prime}-\left\lfloor n^{\prime}\right\rfloor\right) & \text { if } \widehat{\omega}^{\prime}=\left(\left\lfloor s^{\prime}\right\rfloor,\left\lceil n^{\prime}\right\rceil,\left\lfloor e^{\prime}\right\rfloor\right) \\ k \cdot\left(e^{\prime}-\left\lfloor e^{\prime}\right\rfloor\right) & \text { if } \widehat{\omega}^{\prime}=\left(\left\lfloor s^{\prime}\right\rfloor,\left\lfloor n^{\prime}\right\rfloor,\left\lceil e^{\prime}\right\rceil\right) \\ 0 & \text { otherwise. }\end{cases}
$$

If $\omega^{\prime}=Q(\widehat{\omega}, p(\widehat{\omega}))$ is an element of $\nabla\left(\omega^{\prime}\right)$, then

$$
\widehat{Q}\left(\widehat{\omega}^{\prime} \mid \widehat{\omega}, p(\widehat{\omega})\right)= \begin{cases}k \cdot\left(s^{\prime}-\left\lceil s^{\prime}\right\rceil\right) & \text { if } \widehat{\omega}^{\prime}=\left(\left\lfloor s^{\prime}\right\rfloor,\left\lceil n^{\prime}\right\rceil,\left\lceil e^{\prime}\right\rceil\right) \\ k \cdot\left(n^{\prime}-\left\lceil n^{\prime}\right\rceil\right) & \text { if } \widehat{\omega}^{\prime}=\left(\left\lceil s^{\prime}\right\rceil,\left\lfloor n^{\prime}\right\rfloor,\left\lceil e^{\prime}\right\rceil\right) \\ k \cdot\left(e^{\prime}-\left\lceil e^{\prime}\right\rceil\right) & \text { if } \widehat{\omega}^{\prime}=\left(\left\lceil s^{\prime}\right\rceil,\left\lceil n^{\prime}\right\rceil,\left\lfloor e^{\prime}\right\rfloor\right) \\ 0 & \text { otherwise. }\end{cases}
$$

For the first of the two contingencies, the situation is graphically illustrated in Figure 4.1.

\subsubsection{Policy iteration algorithm}

We derive the optimal stationary price policy for the discretized state space, $p: \widehat{\Omega} \rightarrow \mathbb{R}_{+}$. We use the policy iteration algorithm to numerically approximate the optimal stationary price policy for the store.

\section{Policy Iteration Algorithm}

initialize Choose stopping criterion $\varepsilon$ and an arbitrary starting policy $p_{0}: \widehat{\Omega} \rightarrow \mathbb{R}_{+}$. Set $i=1$.

loop 1. Compute the present value from $p_{i-1}$ for each possible starting state in $\widehat{\Omega}$. That is, solve the system of linear equations $V\left(\widehat{\omega}, p_{i-1}\right)=\pi\left(\widehat{\omega}, p_{i-1}(\widehat{\omega})\right)+\delta \sum_{\widehat{\omega}^{\prime} \in \widehat{\Omega}} \widehat{Q}\left(\widehat{\omega}^{\prime} \mid \widehat{\omega}, p_{i-1}(\widehat{\omega})\right) \cdot V\left(\widehat{\omega}^{\prime}, p_{i-1}\right)$.

2. Improve the policy for each state in $\widehat{\Omega}$. That is, solve for each state $\widehat{\omega}$ the problem

$p_{i}(\widehat{\omega}):=\operatorname{argmax}_{z} \pi(\widehat{\omega}, z)+\delta \sum_{\widehat{\omega}^{\prime} \in \widehat{\Omega}} \widehat{Q}\left(\widehat{\omega}^{\prime} \mid \widehat{\omega}, z\right) \cdot V\left(\widehat{\omega}^{\prime}, p_{i-1}\right)$. 
3. Terminate loop if the improvement is negligible:

if $\left\|p_{i}-p_{i-1}\right\|_{\infty}<\varepsilon$ :

then Terminate loop and return $p_{i}$ as the optimal price policy.

else Increase $i$ with one and resume at step 1 .

In step 2 of the loop, given a certain policy, the optimal one-shot deviation with respect to this policy is determined for each possible state. The stationary price policy that is composed from the state prices that induce optimal one-shot improvements guarantees at least the same present value for each possible starting state and, hence, is a better price policy relative to the previous one. This is based on two arguments. First, a one-shot improvement in a state leads to a stationary improvement whenever this state occurs. Second, any improvement implemented in one state implies a weak improvement in all other states. From the one-deviation principle it follows that once no (non-negligible) improvement can be found, an (almost) optimal policy is reached. To sum up, any iteration throughout the running of the policy iteration algorithm guarantees an improvement and, once the algorithm terminates, it returns a (nearly) optimal policy.

In our numerical derivations, we used a bracketing algorithm to numerically solve the maximization problems in step 2 at every iteration. ${ }^{6}$ The procedure underlying this algorithm is analogue to the bisection algorithm for root-solving. Whereas the bisection algorithm starts with two initial points with unequal sign, the bracketing algorithm starts with three initial points (say, $x, y$, and $z$ with $x<y<z$ ) with the property that the middle point $(y)$ has the largest function value. Next, at every iteration, the midpoint of the largest of the two intervals that are determined by the three points (i.e., $[x, y]$ and $[y, z])$ is taken. If the value of the midpoint $(m)$ is less than the value of the middle point $(y)$, then the larger interval is bisected (i.e., $(x, y, z):=(m, y, z)$ or $(x, y, z):=(x, y, m)$ depending on whether the first or the second interval is the largest). Otherwise, the smaller interval is dropped (i.e., $(x, y, z):=(x, m, y)$ or $(x, y, z):=(y, m, z)$ depending on whether the first or the second interval is the largest). This procedure is iterated until the search area has shrunk to a size less than a predefined tolerance level.

One challenge towards implementing the bracketing algorithm is to find three starting points satisfying the property that the middle point implies

\footnotetext{
${ }^{6}$ See (Judd 1998) p. 94.
} 
the largest function value. One starting point $(x)$ we fix at the minimum price of zero. As a second point $(z)$ we take any price sufficiently high for the resulting present value to be either less than the one that results from taking a price of zero or to be below the present value of half that price. Such a price is guaranteed to exist. Finally, we are to search for the middle point $(y)$. Starting in the middle of $x$ and $z$, we half the price until either the present value is above the one that result from taking a price of zero or when the price gets below the predetermined tolerance level for the bracketing algorithm. In the first case, a triple with the desired properties is found and the bracketing algorithm is started; in the second case, a price of zero is optimal in the given state at the given iteration.

\subsection{Model calibration and benchmark outcomes}

In order for the model to make insightful real-life predictions, we need to calibrate the parameters in such a way that it yields static outcomes consistent with real-life data. The model contains two types of parameters. The first type are those whose values are directly retrievable from consumer surveys and financial statements. The second type are not directly retrievable and are calibrated in order for the model outcomes to fit real-life data on market outcomes. We adopt two benchmark scenarios: the situation before P2P was made available to the public and a few years thereafter. More precisely, we take the years 1999 and 2003 for calibration. The year 2003 is chosen because of the abundance of data and the fact that it leaves room for the model to predict market outcomes for later years for which we have the data to verify. The calibration of parameters allows us to analyze, with a reasonable amount of accuracy, the order of magnitude of the societal impacts of the P2P networks on firms' profits and consumers' welfare. Thus, in this model, we speak of numbers in dollar terms.

\subsubsection{Data and evidences}

First of all, we start with the CD prices over the years. According to the aggregate data from (RIAA 2007), the average price of a CD in the U.S. has been kept at around $\$ 14$ over the years. However, the real prices, if one 
takes inflation into account, have been slightly decreasing over the years. In our model, therefore, we should allow the past prices to be slightly higher than the present prices, as long as the average CD price is around $\$ 14$.

Secondly, CD sales. According to the year-end report from (RIAA 2009), the record companies shipped to the consumers, in the year 1999, about 938.9 million copies of $\mathrm{CDs} .{ }^{7}$ That figure has dropped to around 746 million in 2003. This suggests that the CD sales has decreased by about $20 \%$ in 4 years. This implies that in our model, the sales in the store in the fifth period should be about $80 \%$ of those in the first period.

Finally, the P2P network size. When Napster was introduced to the general public in the year 1999, the usage of P2P became instantly wide spread. For simplicity though, we shall take the network size in the year 1999 to be 0 , and assume the network started in the beginning of the year 2000 . Now the question is: how big was the network in 2003? In other words, how many albums are being downloaded via P2P networks in 2003 and beyond? (Blackburn 2004) obtained P2P downloading volume per album from BigChampagne, a company that measures P2P traffic. In the descriptive analysis of the data, it appears that, the average albums were downloaded about 7-10 times more often than they were sold. So the ratio of downloads versus store sales $\frac{n}{s}$ should be around $7-10$.

\subsubsection{Parameter calibration}

In this section we calibrate the model's parameters by assigning real-life values to them. We use existing data and estimates wherever possible, and take the most plausible approximation where we lack real-life evidences. Indeed, with the calibration we do not attempt to achieve pin-point accuracy, but instead aim at a reasonably representative reflection of the music industry. Table 4.1 summarizes the chosen parameter values.

\begin{tabular}{lcrcccccc}
\hline & $\beta$ & \multicolumn{1}{c}{$\gamma$} & $\sigma$ & $\rho$ & $\tau$ & $\lambda$ & $\delta$ & $\mu$ \\
\hline before P2P & $\$ 16$ & $\$-1000$ & $\$ 0.5$ & 0.025 & $\$ 11$ & 0.2 & 0.97 & $\$ 10$ \\
after P2P & $\$ 16$ & $\$ 11$ & $\$ 0.5$ & 0.025 & $\$ 11$ & 0.2 & 0.97 & $\$ 10$ \\
\hline
\end{tabular}

Table 4.1: Calibrated parameter values.

\footnotetext{
${ }^{7}$ See Table 4.5 in Appendix 4.9.1 for an extract of this report.
} 
In the study by (Rob and Waldfogel 2006), surveys suggest that the average valuation of purchased CDs was around $\$ 16$. We therefore take $\beta=16$ in our benchmark calibration. In the same survey, the average valuation of a downloaded album was revealed to be around $\$ 11$. This will lead us to take $\gamma=11$. For the scenario of the year 1999, however, we will set $\gamma=-1000$, since P2P networks were not available. The discount factor $\delta$ can be considered as interest rate, and should be in the realisitc range of $[0.9,1]$. Here we take a modest value of 0.97 , in accordance with the convention of $3 \%$ interest rate. In a study done by (Peitz and Waelbroeck 2005), the authors show a report from IFPI that explains the break-down of the average cost of a CD. This can be estimated to be about $€ 13$ including taxes. However, since in our model we look at all figures in dollar terms, and we know that the CD prices are on average $\$ 14$ in the U.S., a plausible approximation for the per unit cost $\mu$ in our model could be for example $\$ 10$.

The generic cost factor of downloading $\sigma$ and the parameter that captures the curvature of the downloading cost function $\rho$ are model specific and we try to calibrate them in such a way to reflect realistically the downloading costs. As the cost function is $c(n)=\frac{\sigma}{n+\rho}$, we use a calibration with $\rho=0.025$ and $\sigma=\$ 0.5$. This implies that when no one is using the network, it costs $\$ 20$ to download an album, which is more expensive than buying it in the store. However, when $7.5 \%$ of the total market downloads an album, the downloading cost drops significantly to $\$ 5$, which might be tempting for many consumers, though not all. As around half of the market download, the cost drops further towards $\$ 1$, which makes it seriously attractive for everyone. Finally, when the whole population downloads, the cost drops to the minimum of about $\$ 0.5$.

The consumer heterogeneity parameter $\lambda$ is determined by calibration, and is chosen such that both the store and the network achieve realistic amount of quantities in both benchmark scenarios. This leads to $\lambda=0.20$.

The switching cost $\tau$ influences the number of periods it takes for any starting state to converge to the long-run steady state. It takes 4 years (periods) to go from the state of 1999 to the state of 2003. We will therefore choose a value for $\tau$ that yields outcomes that confirm both the situation of 1999 for $\gamma=-1000$ and that of 2003 for $\gamma=11$, and the fact that the transition takes 4 periods. An appropriate value for $\tau$ appears to be 11 . 


\subsection{Model outcomes and analysis}

The numerical method presented in Section 4.3 is programmed into a series of Matlab routines. ${ }^{8}$ We ran the resulting program using the parameter values of Table 4.1. For the discretization, we opt for a grid size of $1 / 20$, i.e. $k=20$; the tolerance level for the price policy to convergence is set to 4 digits after the decimal for the maximum state-wise difference between consecutive iterations. ${ }^{9}$ We obtain outcomes such as price policy, profits, state dynamics, (long-run) probability distribution over states, and welfare.

The triangle of Figure 4.2 in Appendix 4.9.2 displays the optimal price policy in 1999; that is, before the introduction of P2P. The triangulation of the triangle represents the discretized state space for $k=20$. The corner points of this triangle represent the extreme states with full consumer mass at either Store, Network, or Empty. The interior vertices refer to states where the full mass of consumers is divided over the three platforms, the actual division being proportional to the proximity to the corner points. The numbers in the triangle indicate the prices charged by the firm in the different states (231 in total) in the year 1999 before P2P was available. The firm charges higher prices in the states where it has higher market shares, and vice versa, in a monotonic fashion. This seems natural given the presence of the switching cost. Since this case corresponds to $\gamma=-1000$, the value of $s$ is a sufficient state variable for the firm; accordingly, the price policy is a constant on the diagonals from north-west to south-east which represent a particular value of $s$.

Regardless of which distribution of states we start the Markov process from, given the optimal price policy and the induced process of state transition, we always end up in a unique steady state distribution. This is the one depicted in Figure 4.4 in Appendix 4.9.2, where the numbers denote the probability to be in the respective state in the long-run. In this steady state the firm has an expected market share of $23 \%$, the network size is 0 by construction, and the rest of the market $(77 \%)$ is unserved. The expected steady-state price charged by the firm is $\$ 14.60$.

\footnotetext{
${ }^{8}$ The Matlab routines are available upon request.

${ }^{9}$ The tolerance level of the bracketing algorithm for policy improvement is set to 6 digits after the decimal.
} 
Figure 4.3 in Appendix 4.9.2 presents the firm's optimal pricing policy after the introduction of $\mathrm{P} 2 \mathrm{P}$. With the exception of the situation when the network size is 0 , the firm still charges higher prices in states where its market share is higher. Moreover, for the same market share of the firm, the firm charges in general a higher price when the network size is larger. This is perhaps due to the fact that it is more difficult and less profitable to attract consumers from the network than from the unserved market, hence the pricing strategy is less aggressive. The prices charged in the presence of $\mathrm{P} 2 \mathrm{P}$ are in general lower than before $\mathrm{P} 2 \mathrm{P}$ was introduced.

Figures 4.4-4.10 in Appendix 4.9.2 show the evolution and convergence of market shares resulting from the introduction of $\mathrm{P} 2 \mathrm{P}$. These market share dynamics are obtained from following the Markov process induced by the optimal price policy of the situation after the introduction of P2P (Figure 4.3) starting from the steady state distribution of the situation before the introduction of P2P (Figure 4.4). Every iteration represents precisely one state transition and, therefore, one year in reality. The figures indicate that the mass of consumers first stirs slightly at the starting state and then gradually shifts towards a new steady state (Figure 4.10). It takes 20 years (periods) to converge to within $1 \%$ of the steady state. However, the market share division of 2008 (Figure 4.9) is in fact already close to the steady state. The price in the steady state is about $\$ 13.73$ and the firm's market share drops to $6 \%$, while the network size increases to about 75\%: more than 10 times the CD sales quantity; only $19 \%$ of the market remains unserved.

Table 4.2 provides some additional insights into the dynamics of the static model outcomes. The columns are arranged in years, where "Std.St." stands for the steady state. The rows show the model outcomes, where $p$, $\pi, c s$, and $w$ stand for price, profit, consumers' surplus, and welfare, respectively. Notice that the numbers refer to weighted averages of the state outcomes, where the weight on a particular state is the probability that this state is visited in the respective year. All numbers except for the prices are obtained by multiplying the values of the model outcomes with a factor of 4.1 billion. This scaler is estimated by taking the ratio between the total CD sales in the U.S., according to the RIAA's year end report, and the CD sales quantity $s$ in our model.

The years 1999 and 2003 were used to calibrate some of the model's pa- 
Chapter 4. A dynamic point of view

\begin{tabular}{lrrrrrrr}
\hline & \multicolumn{1}{c}{1999} & \multicolumn{1}{c}{2000} & \multicolumn{1}{c}{2001} & \multicolumn{1}{c}{2002} & \multicolumn{1}{c}{2003} & \multicolumn{1}{c}{2008} & \multicolumn{1}{c}{ Std.St. } \\
\hline$p$ (\$) & 14.60 & 14.02 & 14.17 & 14.20 & 14.19 & 13.88 & 13.73 \\
$s$ (billions) & 0.9377 & 1.0262 & 1.0025 & 0.9049 & 0.7843 & 0.3743 & 0.2312 \\
$n$ (billions) & 0 & 0.0554 & 0.2517 & 0.6408 & 1.0951 & 2.5728 & 3.0787 \\
$\pi(\$$ bn) & 4.3206 & 3.9844 & 3.7134 & 3.2480 & 2.7679 & 1.3370 & 0.8655 \\
$c s(\$$ bn) & 16.6120 & 18.8030 & 22.0781 & 26.6213 & 31.2834 & 45.1558 & 49.7650 \\
$w(\$$ bn) & 20.9330 & 22.7874 & 25.7915 & 29.8693 & 34.0513 & 46.4928 & 50.6305 \\
\hline
\end{tabular}

Table 4.2: Evolution of market outcomes over years.

rameters; so the match with real-life figures in these two periods is quite accurate. The remaining numbers in the table are outcomes of the model, but nevertheless provide quite a good fit to the real-life figures (as far as they are available). Perhaps surprisingly, the outcomes not only reflect the general decreasing trend of CD prices and sales as in reality very well, but capture the anomalies of the CD sales increase in 2000 and the CD price increase in 2001 and 2002 as well.

Now, let us scrutinize some of the key numbers across the years. The firm's profit decreases steadily over the years. By the year 2003, this decrease had led to a huge loss of $\$ 1.6$ billion in annual profits, according to the model's estimation. As (RIAA 2009) suggests that the CD sales revenue dropped from around $\$ 12.8$ billion in the highest year 1999 to around $\$ 11.2$ billion in 2003 (see Table 4.5 in Appendix 4.9.1), this profit estimate seems to be in the right range. Consumers' surplus, however, increases in the same period by some $\$ 14.7$ billion per annum. As a result of that, total welfare benefits by around $\$ 13.1$ billion per annum.

So, what does the model predict for the market outcomes for the year 2008 then? This is as far as the data is available, which enables us to verify the accuracy of the predictions. According to the model estimations, the price drops further, CD sales too drop to 374.3 million copies, and the network size continues to grow to around 2.6 billion copies. The official figure from RIAA being 384.7 million copies suggests that, despite various factors in the economy that could influence the CD sales, our model is doing a good job in predicting market outcomes beyond the year 2003. The figures in Table 4.2 suggest a further drop in annual profit by about $\$ 1.4$ billion compared to 2003 , and that makes it nearly $\$ 3$ billion less than in 1999 . Meanwhile, not 
surprisingly, there is a further increase in total welfare by about $\$ 12.4$ billion per annum, comparing to 2003. Finally, in the long-run steady state (around the year 2020), the price will stay around $\$ 13.7$, the CD sales will hoover around 231.2 million, the profit will settle down to around $\$ 865.5$ million, while the total welfare will be around $\$ 29.7$ billion per annum more than in 1999. For more predictions on market outcomes (between 2009-2020), see Table 4.6 in Appendix 4.9.1.

\subsection{Comparative statics}

The previous section focussed on the market outcomes across the years 1999 to 2008 and made predictions on market outcomes for the years beyond 2008 in the steady state. This section extends the analysis towards the effects of small changes in the parameters on the steady state market outcomes. Table 4.4 presents the comparative statics at the steady state. The numbers in the cells refer to the elasticities of market outcomes (in rows) with respect to the model's parameters (in columns). As a reference point, the comparative statics of the benchmark's starting state in 1999 without P2P are included Table 4.3. In this way, the impact of $\mathrm{P} 2 \mathrm{P}$ on certain comparative statics becomes apparent.

\begin{tabular}{lrrrr}
\hline & \multicolumn{1}{c}{$\beta$} & \multicolumn{1}{c}{$\tau$} & \multicolumn{1}{c}{$\lambda$} & \multicolumn{1}{c}{$\mu$} \\
\hline$p$ & 0.2632 & -0.1665 & -0.3829 & 0.5293 \\
$s$ & 2.7418 & -0.9403 & 0.0868 & -1.7097 \\
$e$ & -0.8131 & 0.2789 & -0.0258 & 0.5070 \\
$\pi$ & 3.5843 & -1.4586 & -1.1266 & -2.1913 \\
$c s$ & 0.9945 & -0.1298 & -1.1581 & -0.6232 \\
$w$ & 1.5291 & -0.4041 & -1.1528 & -0.9469 \\
\hline
\end{tabular}

Table 4.3: Comparative statics of steady state outcomes without P2P.

The intuition behind the numbers in Table 4.3 compares well to standard intuitions of a monopoly analysis. Meanwhile, according to Table 4.4, the model outcomes are generally most sensitive towards changes in the parameters $\beta, \gamma$, and $\mu$. In particular, CD sales quantity and the firm's profit increase substantially in $\beta$ and decrease in $\gamma$ and $\mu$. This seems fairly in- 
Chapter 4. A dynamic point of view

\begin{tabular}{lrrrrrrr}
\hline & \multicolumn{1}{c}{$\beta$} & \multicolumn{1}{c}{$\gamma$} & \multicolumn{1}{c}{$\sigma$} & \multicolumn{1}{c}{$\rho$} & \multicolumn{1}{c}{$\tau$} & \multicolumn{1}{c}{$\lambda$} & \multicolumn{1}{c}{$\mu$} \\
\hline$p$ & -0.0815 & 0.1459 & -0.0033 & 0.0002 & -0.0717 & -0.3707 & 0.8517 \\
$s$ & 4.6613 & -3.2408 & 0.1815 & -0.0096 & -0.4208 & -1.3290 & -3.0482 \\
$n$ & -0.3365 & 0.8674 & -0.0517 & 0.0026 & -0.2115 & 0.4559 & 0.2217 \\
$e$ & -0.0531 & -2.4314 & 0.1483 & -0.0073 & 0.9474 & -1.3876 & 0.0285 \\
$\pi$ & 4.4888 & -2.8493 & 0.1742 & -0.0089 & -0.6790 & -2.7032 & -2.7093 \\
$c s$ & -0.1191 & 1.0267 & -0.1314 & -0.0496 & -0.2162 & -0.2354 & 0.0172 \\
$w$ & -0.0403 & 0.9604 & -0.1262 & -0.0489 & -0.2241 & -0.2775 & -0.0294 \\
\hline
\end{tabular}

Table 4.4: Comparative statics of steady state outcomes with P2P.

tuitive, as $\beta$ and $\gamma$ represent the valuation of the $\mathrm{CD}$ and the downloads, and $\mu$ the cost of producing one CD. Less intuitive is the fact that higher per unit cost $\mu$ increases consumers' surplus. This is due to the fact that a higher production cost induces a higher price, which encourages consumers to download and consequently implies a gain in consumers' surplus because of positive downloading externalities. Total welfare, however, does suffer from high costs, which implies that the gain in consumers' surplus is not sufficient to compensate for the loss in the firm's profit. As one would expect, this effect does not exist in the case before P2P was introduced (see Table 4.3).

The effect of $\beta$ is exactly the opposite as the effect of $\gamma$ on all outcomes except for the unserved market $e$ : both a higher $\beta$ and a higher $\gamma$ make the unserved market smaller. The intuition for $\beta$ and $\gamma$ generally having an opposite effect is quite obvious, with the exception of the negative effect of the valuation of CD on consumers' surplus and total welfare. This effect can be explained by the fact that a higher valuation of CD induces consumers to switch from the P2P network to the store, thereby making it more costly to download for those downloaders who stay in the network (network effect). Again, without the presence of $\mathrm{P} 2 \mathrm{P}, \beta$ naturally has a positive effect on the consumers' surplus. A more careful look at the effects of $\beta$ and $\gamma$ on the $\mathrm{CD}$ price reveals even more trickeries. A higher valuation of $\mathrm{CD}$ decreases the $\mathrm{CD}$ price while a higher valuation of downloads increases it. As we will explain in more detail little later on, this is due to the price elasticity of demand of CDs given the convex functional form of the cost of downloading.

Although the model outcomes are relatively inelastic with respect to the 
switching $\operatorname{cost} \tau$, with the exception of the size of the unserved market $e$, a higher $\tau$ induces a decrease in all of the predicted market outcomes. In particular, switching costs have a negative effect on the prices indicating that, for the calibrated values, our model predicts the "investment effect" to dominate the "harvesting effect". ${ }^{10}$ A higher switching cost deters consumers from consuming any product and forces the firm to charge a lower price, resulting in less consumers' surplus and profit. This holds true for the situation with $\mathrm{P} 2 \mathrm{P}$ as well as the situation without $\mathrm{P} 2 \mathrm{P}$.

Consumers' taste heterogeneity is captured by the parameter $\lambda$ : a larger $\lambda$ implies lower heterogeneity. Both tables reveal that a decrease in consumers' heterogeneity $\lambda$ leads to a lower price and a smaller size of the unserved market $e$. In the situation without $\mathrm{P} 2 \mathrm{P}$, despite the fact that these extra consumers go to the store, the profit suffers. This is caused by the large decrease in price that is needed to accommodate the lower heterogeneity. In the situation with $\mathrm{P} 2 \mathrm{P}$, despite the lower price charged by the firm, the extra consumers go to the network, together with some consumers that switch from the store. Both negative effects on prices and sales lead to a decreased profit. Evidentally, high heterogeneity complicates the formation of the P2P network and hence is of benefit to the firm. Total welfare in both situations (with and without $\mathrm{P} 2 \mathrm{P}$ ) is negatively affected by a decrease in heterogeneity, though the effect of $\lambda$ on consumers' surplus should be interpreted with caution, since $\lambda$ determines the sizes of the shocks in utility of all consumers, and as such directly affects consumers' surplus.

The generic cost factor of downloading $\sigma$ has different properties than the switching cost $\tau$. In fact, it has the exact opposite effects as $\gamma$, the valuation of downloads. Higher $\sigma$ implies higher downloading cost for everyone, making P2P less attractive to consumers, similar to the effects of lower $\gamma$. However, the interpretation of $\sigma$ and $\gamma$ are quite different, which makes the implications different as well. The quality parameter $\gamma$ measures the value of a download to a consumer regardless of how the download is acquired. Thus, $\gamma$ only reflects the intrinsic quality of the download copy, e.g. in the case of music, the sound quality. It does not reflect the fact that the copy has to be illegally downloaded and that it costs time and effort. That is what the cost factor $\sigma$ captures. Enforcement of intellectual property rights relates

\footnotetext{
${ }^{10}$ See (Cabral 2009).
} 
to an increased value of $\sigma$ (consumers face a high risk of punishment when using P2P). Table 4.4 shows that a higher $\sigma$ improves the profit of the firm and decreases consumers' surplus and welfare. The total welfare suffers due to the increased size of the unserved market $e$ and the sheer downloading costs that are incurred for those who do download. In other words, a social planner who aims at maximizing welfare should be careful in enforcing intellectual property rights in the context of $\mathrm{P} 2 \mathrm{P}$ file sharing, considering the welfare enhancing effect of $\mathrm{P} 2 \mathrm{P}$ shown in this crude yet vivid demonstration.

Furthermore, an increase in $\sigma$ does not lead to an increase in the price of CDs as intuition would suggest, since high $\sigma$ harms the attractiveness of the P2P network, with which the firm competes in price. In fact, if anything, the price decreases slightly in $\sigma$. This is attributable to the price elasticity of demand for CDs, just as for the effects of $\beta$ and $\gamma$ on the price. To put it simply, when $\sigma$ increases, the P2P network becomes less attractive and there are a lot more potential consumers out there for the firm to win over, and hence it charges a slightly lower price to attract these switching consumers. Conversely, when $\sigma$ decreases, $\mathrm{P} 2 \mathrm{P}$ becomes more attractive and a big chunk of consumers will go to the network, making the remaining consumers in the store relatively inelastic to prices, and hence the higher price. Ultimately, this pricing scheme is caused by the convex form of the downloading cost function with respect to the network size, $c(n)=\frac{\sigma}{\rho+n}$. This functional form induces strong network externalities when the network is relatively small and weak network externalities when the network is large.

\subsection{Discussion}

The data and estimates in this chapter have ignored the sales of legally downloaded songs and albums from online music stores. Indeed, with the introduction of Apple's popular iTunes Music Store in 2004, music sales through digital channel have soared, crowding out to some extent the traditional CD sales. ${ }^{11}$

It can be argued, therefore, that the loss of music sales over the relevant years in this chapter may be overestimated due to the fact that the param-

\footnotetext{
${ }^{11}$ See (RIAA 2009).
} 
eter calibrations in this model does not take into account legal downloads which are plausible substitutes to physical CDs. However, the addition of legal downloads into the model as an extra ingredient is not so straightforward, because it would add another dimension to the state space. Another way to tackle the problem would be to ignore the sales of physical CDs and focus on the battle between P2P downloads and legal downloads alone. In that case, the P2P network would be the incumbent and the online music store the new entrant, although there would be some problems concerning the calibrations of certain parameters since by the time online music store entered the market P2P was not yet at its steady-state size. Consequently, we choose to ignore the effect that legal downloads have had on the music market and focus instead on the long-standing battle between the physical CDs and piracy via P2P.

Although it is beyond the analysis of this chapter, it is worth digressing on the issue of dynamic efficiency. In other words, the very purpose of intellectual property rights law is to protect the creative incentives of the content creators by granting them exclusive rights to distribute and to sell their creations, and in so doing to recuperate the costs of creation and hence to encourage more future creations. Piracy of intellectual properties such as patents or copyrighted materials for the purpose of reselling and regenerating illegal profit for the copier undoubtedly hurts the creative incentives of the content provider. However, how much are the creative incentives hurt by $\mathrm{P} 2 \mathrm{P}$ file-sharing, while no one is reselling the products and hence little profit is redistributed? One should not forget that the content creators and the intellectual property rights owners are very often different entities - the former being the artists and the latter being the publishing firms. Damages incurred to IPR owners do not necessarily imply damages incurred to content creators, and it is the latters' creative incentives that matter. Is it not potentially very profitable to reach an otherwise impossibly large audience even if that means one has to give away some music for free?

After all, the Web 2.0 concept (community-based web environments such as Facebook, Youtube, and Myspace) encourages new content creation by the average public on such a massive scale that the traditional media could never have dreamed of. Yet, rather than fostering such creative activities, the existing copyright laws to a large extent curb them by means of preven- 
tion and prohibition of the use of existing artistic materials for further creations. One may wonder whether a new form of IPR (especially copyrights) law is needed in this fast-paced information-based society. Perhaps one that is much more relaxed with regard to the fair use of copyrighted materials.

\subsection{Concluding remarks}

This chapter uses a dynamic model to solve the optimal dynamic and statedependent pricing strategy for a firm that releases music CDs and sells them in the store to compete with the P2P file-sharing networks on the internet.

An interesting point of this model is that we can observe the dynamics of network formation. We can also observe exactly how the firm sets its price conditional on its market share and the network size. Sometimes it sets the price very low in order to win vital market shares to fight against the P2P network, while other times accommodates the network by setting a high price to reap the profit from its own customers.

Based on data from 1999 and 2003, the model predicts the sales quantity of the year 2008 rather accurately to be around 374.3 million copies, while the real figure is 384.7 million. It also predicts that the CD sales will have dropped to around 231.2 million copies per year around the year 2020, when according to the model estimations the long-run steady state is approximately reached. More importantly, our model is able to estimate the welfare gain over the years brought by the P2P file-sharing networks. In the year 2003, four years after P2P was introduced, while the music industry was suffering from a $\$ 1.6$ billion forgone profit per year, the total welfare was up by $\$ 13.1$ billion per year. By the year 2008 , the industry profit has dropped by a further $\$ 1.3$ billion, and welfare soared by a further $\$ 12.4$ billion per year, making it $\$ 25.6$ billion more than in 1999 . In the long-run steady state (around year 2020), the industry profit will have dropped to a mere $\$ 865.5$ million per year, and total welfare are set to have improved since 1999 by a whopping $\$ 29.7$ billion per year.

In the comparative static analysis of the model, one of our most important findings is that total welfare is negatively related to the generic cost factor of downloading. This result coincides with the findings in Chapter 2, 3 and by (Rob and Waldfogel 2006), in the sense that the existence of P2P ac- 
tually enhances total welfare. This implies that by making file-sharing more difficult for the consumers, the government is effectively curbing the society from enjoying a high welfare level that $\mathrm{P} 2 \mathrm{P}$ technology would generate.

Other findings include an interesting pricing scheme of the firm in the sense that the steady state price decreases when its product becomes more attractive (higher valuation of the $\mathrm{CD}$ or higher generic cost factor of downloading), and increases in the valuation of the competing product (higher valuation of the downloads and lower generic cost factor of downloading). Like in Chapter 2 and 3, this is due to the convex functional form of the cost of downloads, which affects the price elasticity of demand of the CDs.

Finally, it must be clarified that the results of this chapter by no means support the infringement of intellectual property rights or piracy in general. There is little doubt that the industrial profits of information goods have suffered from the presence of P2P file-sharing, although to different extents depending on the specific industries. In the case of music CDs, the monumental drop in sales revenue of CDs over the last ten years is astonishing. On the other hand, thanks to the colossal amount of pirated music that serves the otherwise unserved consumers, the immense gain on total welfare is just as impossible to ignore. The next interesting question then is how dynamic efficiency is affected. When are artists' creative incentives hurt so much that new creations are hindered by file-sharing? An entirely different model of dynamic efficiency would be needed to answer that questions. The model would have to capture the conflict of interests between the publisher and the artist, whilst keeping an accurate measurement for the quality and quantity of the new creations. It would also have to take into account consumers' valuation for product variety. Alternatively, one can investigate this question empirically, by measuring all these variables for the music products over the past years and search for signs of improvement or deterioration. 


\subsection{Appendix}

\subsubsection{Tables}

\begin{tabular}{lrrrrc}
\hline & 1999 & \multicolumn{1}{c}{2000} & \multicolumn{1}{c}{2001} & \multicolumn{1}{c}{2002} & 2003 \\
\hline units (billions) & 0.9389 & 0.9425 & 0.8819 & 0.8033 & 0.7460 \\
value (\$billions) & 12.8163 & 13.2145 & 12.9094 & 12.0441 & 11.2329 \\
\hline & 2004 & 2005 & 2006 & 2007 & 2008 \\
\hline units (billions) & 0.7670 & 0.7054 & 0.6197 & 0.5111 & 0.3847 \\
value (\$billions) & 11.4465 & 10.5202 & 9.3726 & 7.4523 & 5.4713 \\
\hline
\end{tabular}

Table 4.5: RIAA 2008 Year-End U.S. CD sales statistics.

\begin{tabular}{lrrrrrr}
\hline & 2009 & 2010 & 2011 & 2012 & \multicolumn{1}{c}{2013} & 2014 \\
\hline$p$ (\$) & 13.84 & 13.81 & 13.79 & 13.77 & 13.76 & 13.75 \\
$s$ (billions) & 0.3374 & 0.3100 & 0.2895 & 0.2743 & 0.2628 & 0.2546 \\
$n$ (billions) & 2.7031 & 2.8007 & 2.8733 & 2.9270 & 2.9668 & 2.9963 \\
$e$ (billions) & 1.0594 & 0.9893 & 0.9373 & 0.8987 & 0.8700 & 0.8491 \\
\hline & 2015 & 2016 & 2017 & 2018 & 2019 & 2020 \\
\hline$p$ (\$) & 13.75 & 13.74 & 13.74 & 13.73 & 13.73 & 13.73 \\
$s$ (billions) & 0.2485 & 0.2440 & 0.2407 & 0.2382 & 0.2362 & 0.2349 \\
$n$ (billions) & 3.0180 & 3.0340 & 3.0459 & 3.0545 & 3.0611 & 3.0660 \\
$e$ (billions) & 0.8335 & 0.8221 & 0.8134 & 0.8073 & 0.8028 & 0.7991 \\
\hline
\end{tabular}

Table 4.6: Some predictions of market outcomes in the future. 


\title{
4.9.2 Triangles
}

\author{
$E$ \\ 12.70 \\ 13.2312 .70 \\ $\begin{array}{llll}13.71 & 13.23 & 12.70\end{array}$ \\ $\begin{array}{llll}14.07 & 13.71 & 13.23 & 12.70\end{array}$ \\ $\begin{array}{lllll}14.43 & 14.07 & 13.71 & 13.23 & 12.70\end{array}$ \\ $\begin{array}{llllll}14.72 & 14.43 & 14.07 & 13.71 & 13.23 & 12.70\end{array}$ \\ $\begin{array}{lllllll}14.97 & 14.72 & 14.43 & 14.07 & 13.71 & 13.23 & 12.70\end{array}$ \\ $\begin{array}{lllllllll}15.14 & 14.97 & 14.72 & 14.43 & 14.07 & 13.71 & 13.23 & 12.70\end{array}$ \\ $\begin{array}{lllllllll}15.31 & 15.14 & 14.97 & 14.72 & 14.43 & 14.07 & 13.71 & 13.23 & 12.70\end{array}$ \\ $\begin{array}{lllllllllll}15.46 & 15.31 & 15.14 & 14.97 & 14.72 & 14.43 & 14.07 & 13.71 & 13.23 & 12.70\end{array}$ \\ $\begin{array}{lllllllllll}15.55 & 15.46 & 15.31 & 15.14 & 14.97 & 14.72 & 14.43 & 14.07 & 13.71 & 13.23 & 12.70\end{array}$ \\ $\begin{array}{lllllllllllll}15.66 & 15.55 & 15.46 & 15.31 & 15.14 & 14.97 & 14.72 & 14.43 & 14.07 & 13.71 & 13.23 & 12.70\end{array}$ \\ $\begin{array}{lllllllllllll}15.75 & 15.66 & 15.55 & 15.46 & 15.31 & 15.14 & 14.97 & 14.72 & 14.43 & 14.07 & 13.71 & 13.23 & 12.70\end{array}$ \\ $\begin{array}{llllllllllllll}15.82 & 15.75 & 15.66 & 15.55 & 15.46 & 15.31 & 15.14 & 14.97 & 14.72 & 14.43 & 14.07 & 13.71 & 13.23 & 12.70\end{array}$ \\ $\begin{array}{lllllllllllllllll}15.89 & 15.82 & 15.75 & 15.66 & 15.55 & 15.46 & 15.31 & 15.14 & 14.97 & 14.72 & 14.43 & 14.07 & 13.71 & 13.23 & 12.70\end{array}$ \\ $\begin{array}{llllllllllllllll}15.95 & 15.89 & 15.82 & 15.75 & 15.66 & 15.55 & 15.46 & 15.31 & 15.14 & 14.97 & 14.72 & 14.43 & 14.07 & 13.71 & 13.23 & 12.70\end{array}$ \\ $\begin{array}{llllllllllllllllll}16.01 & 15.95 & 15.89 & 15.82 & 15.75 & 15.66 & 15.55 & 15.46 & 15.31 & 15.14 & 14.97 & 14.72 & 14.43 & 14.07 & 13.71 & 13.23 & 12.70\end{array}$ \\ $\begin{array}{llllllllllllllllllll}16.06 & 16.01 & 15.95 & 15.89 & 15.82 & 15.75 & 15.66 & 15.55 & 15.46 & 15.31 & 15.14 & 14.97 & 14.72 & 14.43 & 14.07 & 13.71 & 13.23 & 12.70\end{array}$ \\ $\begin{array}{llllllllllllllllllllll}16.09 & 16.06 & 16.01 & 15.95 & 15.89 & 15.82 & 15.75 & 15.66 & 15.55 & 15.46 & 15.31 & 15.14 & 14.97 & 14.72 & 14.43 & 14.07 & 13.71 & 13.23 & 12.70\end{array}$ \\ $\begin{array}{llllllllllllllllllllllll}16.13 & 16.09 & 16.06 & 16.01 & 15.95 & 15.89 & 15.82 & 15.75 & 15.66 & 15.55 & 15.46 & 15.31 & 15.14 & 14.97 & 14.72 & 14.43 & 14.07 & 13.71 & 13.23 & 12.70\end{array}$ \\ $\begin{array}{llllllllllllllllllllll}16.17 & 16.13 & 16.09 & 16.06 & 16.01 & 15.95 & 15.89 & 15.82 & 15.75 & 15.66 & 15.55 & 15.46 & 15.31 & 15.14 & 14.97 & 14.72 & 14.43 & 14.07 & 13.71 & 13.23 & 12.70\end{array}$
}

Figure 4.2: Stationary optimal pricing policy before P2P. 
$E$

12.27

$\begin{array}{lll}12.28 & 12.93\end{array}$

$\begin{array}{llll}12.78 & 13.47 & 12.86\end{array}$

$\begin{array}{llll}13.22 & 13.80 & 13.42 & 12.80\end{array}$

$\begin{array}{llllll}13.80 & 14.18 & 13.88 & 13.39 & 12.84\end{array}$

$\begin{array}{lllllll}13.75 & 14.49 & 14.20 & 13.84 & 13.37 & 12.82\end{array}$

$\begin{array}{lllllll}14.04 & 14.66 & 14.48 & 14.19 & 13.84 & 13.38 & 12.80\end{array}$

$\begin{array}{llllllll}14.36 & 14.90 & 14.70 & 14.42 & 14.19 & 13.85 & 13.45 & 12.77\end{array}$

$\begin{array}{lllllllll}14.95 & 15.07 & 14.84 & 14.64 & 14.45 & 14.20 & 13.87 & 13.45 & 12.76\end{array}$

$\begin{array}{llllllllll}14.61 & 15.21 & 14.96 & 14.81 & 14.62 & 14.46 & 14.22 & 13.88 & 13.47 & 12.74\end{array}$

$\begin{array}{lllllllllll}14.80 & 15.27 & 15.09 & 14.95 & 14.78 & 14.62 & 14.46 & 14.23 & 13.97 & 13.48 & 12.71\end{array}$

$\begin{array}{llllllllllll}15.34 & 15.40 & 15.19 & 15.04 & 14.91 & 14.78 & 14.66 & 14.49 & 14.27 & 13.99 & 13.50 & 12.69\end{array}$

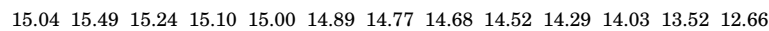

$\begin{array}{llllllllllllll}15.16 & 15.51 & 15.31 & 15.18 & 15.07 & 14.99 & 14.90 & 14.80 & 14.71 & 14.55 & 14.34 & 14.06 & 13.54 & 12.63\end{array}$

$\begin{array}{lllllllllllllll}15.12 & 15.58 & 15.39 & 15.22 & 15.14 & 15.06 & 15.00 & 14.91 & 14.81 & 14.73 & 14.59 & 14.41 & 14.10 & 13.57 & 12.73\end{array}$

$\begin{array}{lllllllllllllllll}15.20 & 15.67 & 15.42 & 15.29 & 15.18 & 15.13 & 15.06 & 15.01 & 14.92 & 14.87 & 14.77 & 14.62 & 14.46 & 14.15 & 13.60 & 12.71\end{array}$

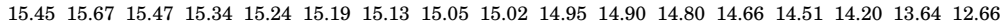

$\begin{array}{llllllllllllllllllll}15.30 & 15.72 & 15.52 & 15.36 & 15.30 & 15.23 & 15.19 & 15.12 & 15.09 & 15.04 & 14.98 & 14.93 & 14.84 & 14.70 & 14.56 & 14.25 & 13.69 & 12.61\end{array}$

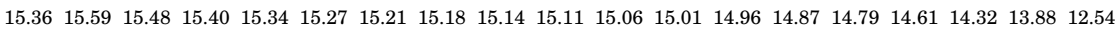

$\begin{array}{lllllllllllllllllllllll}15.68 & 15.63 & 15.52 & 15.44 & 15.36 & 15.32 & 15.25 & 15.23 & 15.19 & 15.15 & 15.13 & 15.09 & 15.03 & 15.00 & 14.92 & 14.84 & 14.67 & 14.38 & 13.96 & 12.44\end{array}$

$\begin{array}{llllllllllllllllllllll}15.51 & 15.66 & 15.55 & 15.46 & 15.39 & 15.31 & 15.29 & 15.26 & 15.24 & 15.20 & 15.17 & 15.16 & 15.12 & 15.09 & 15.03 & 14.96 & 14.89 & 14.73 & 14.47 & 14.02 & 12.32\end{array}$

$S$

Figure 4.3: Stationary optimal pricing policy with P2P.

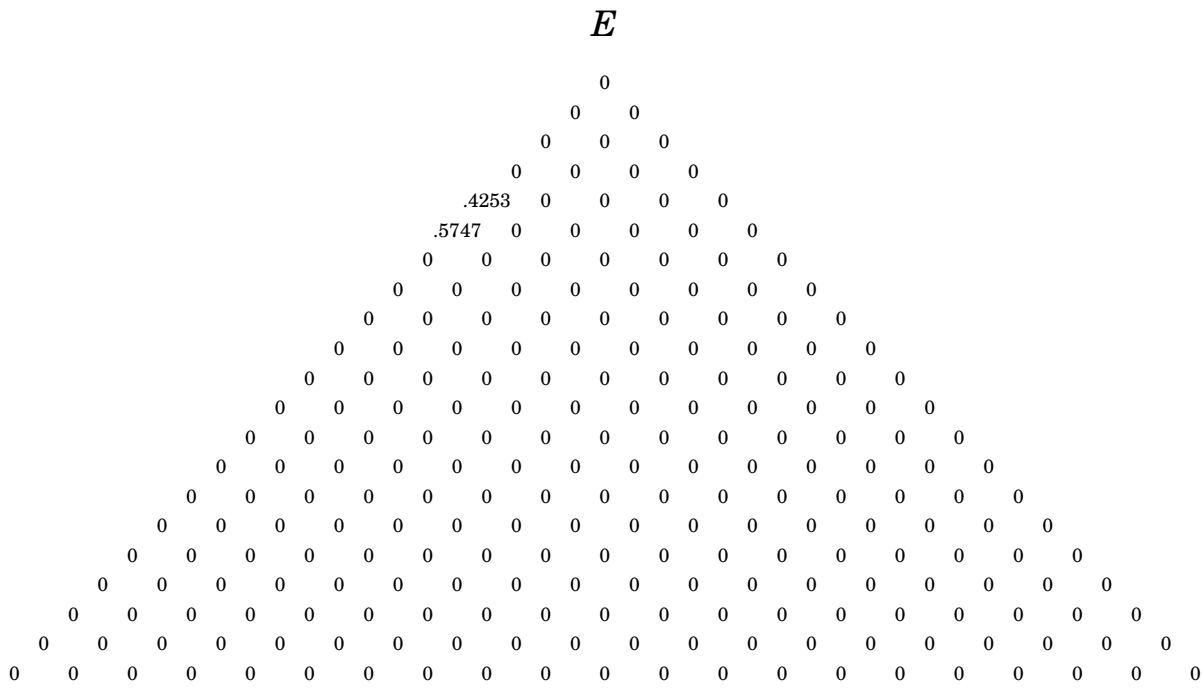

Figure 4.4: Probability distribution over states in steady state just before the introduction of P2P (1999). 


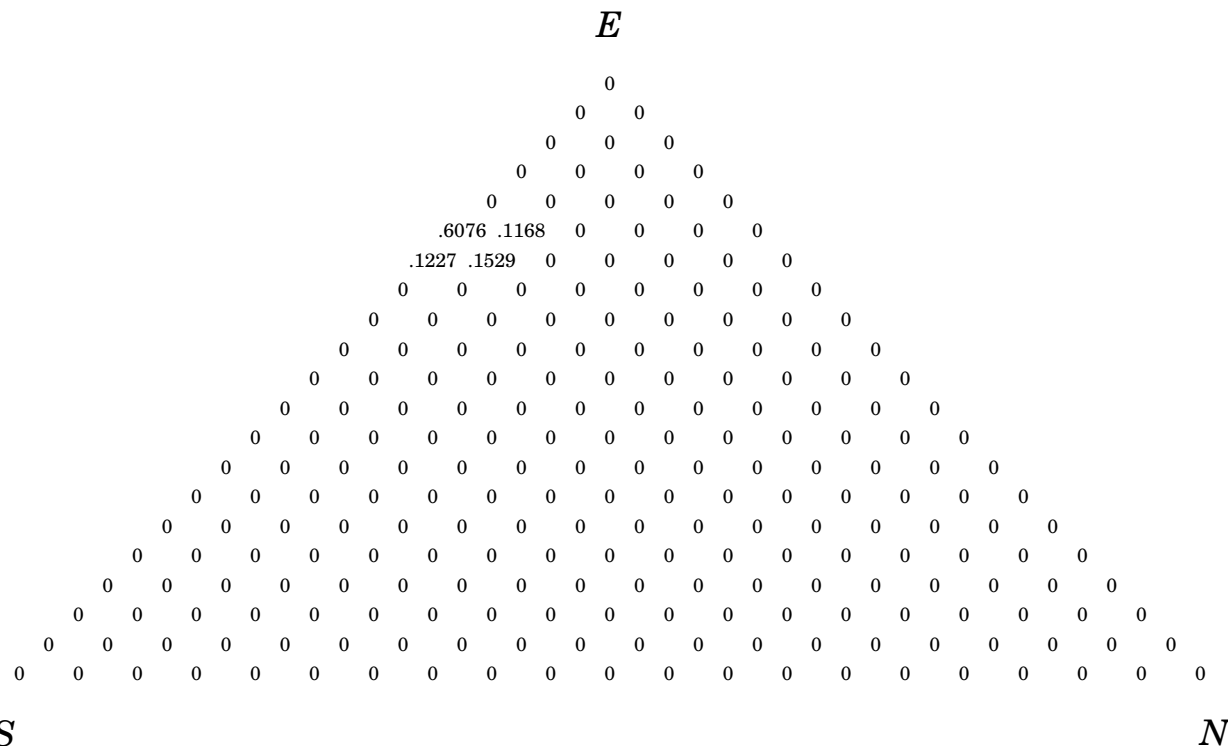

Figure 4.5: Probability distribution over states one year after the introduction of P2P (2000).

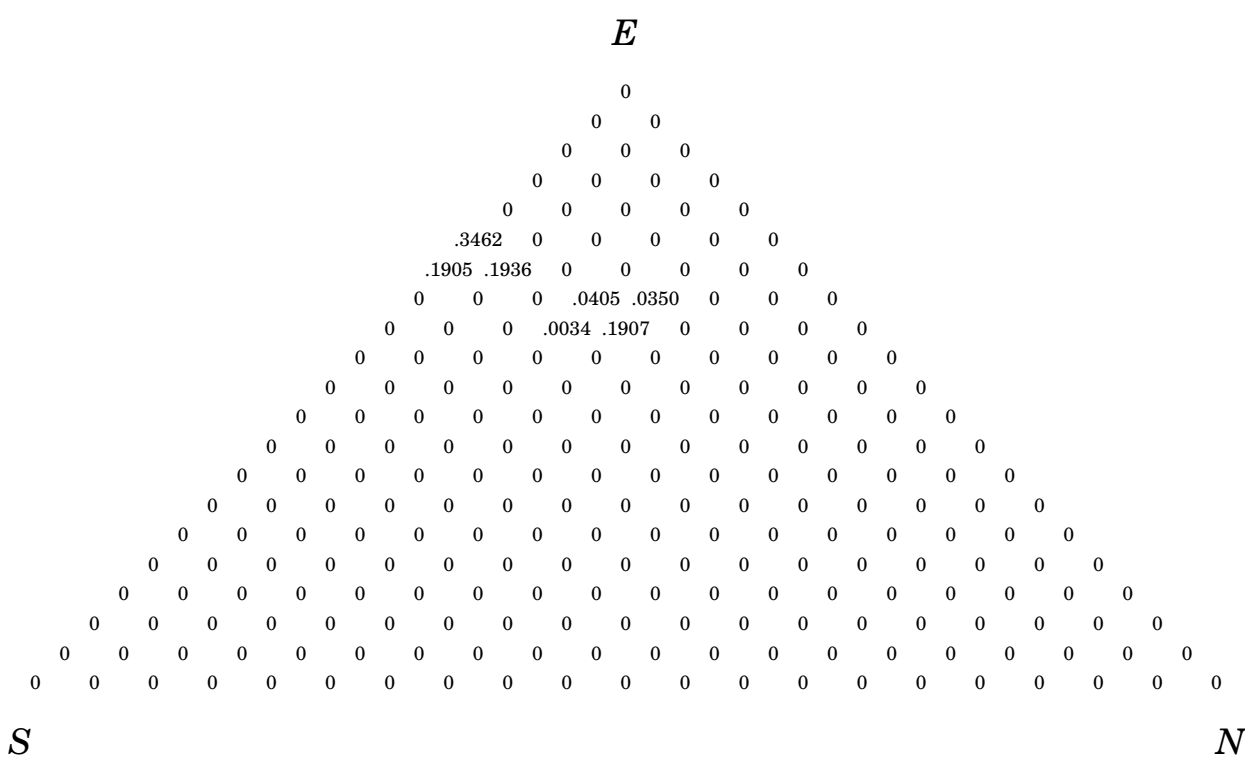

Figure 4.6: Probability distribution over states two years after the introduction of P2P (2001). 


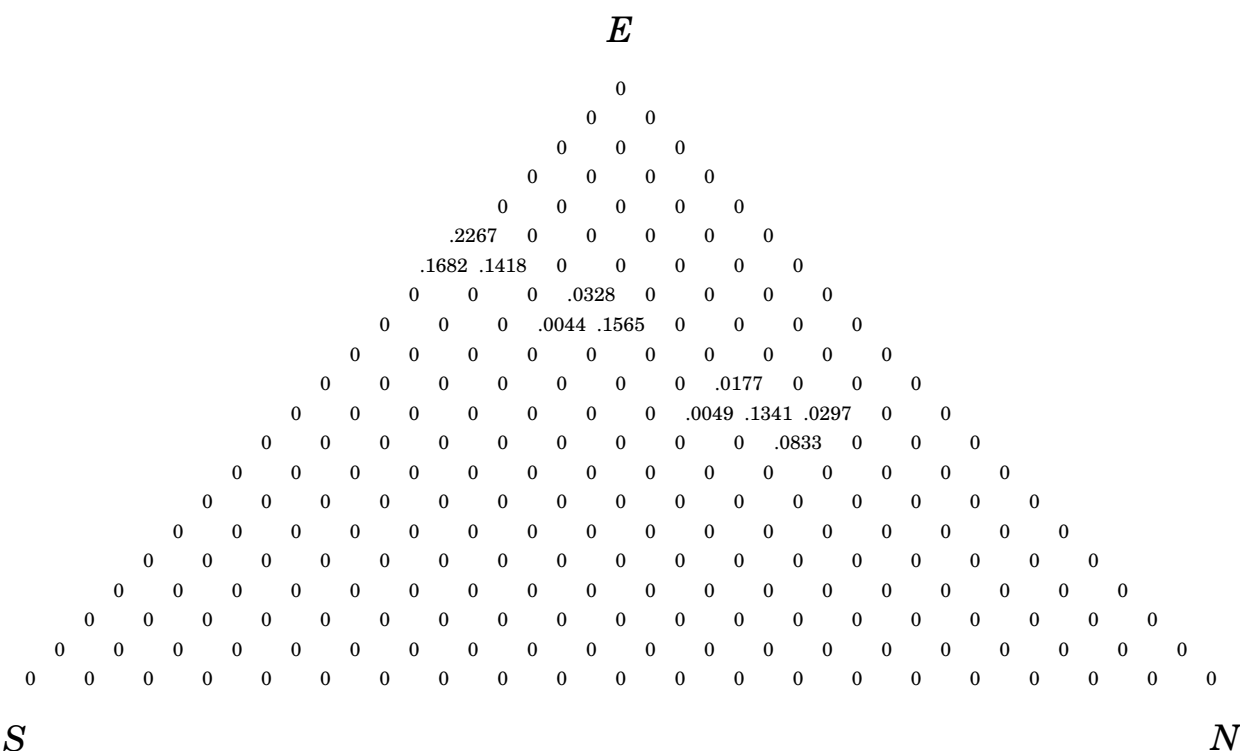

Figure 4.7: Probability distribution over states three years after the introduction of P2P (2002).

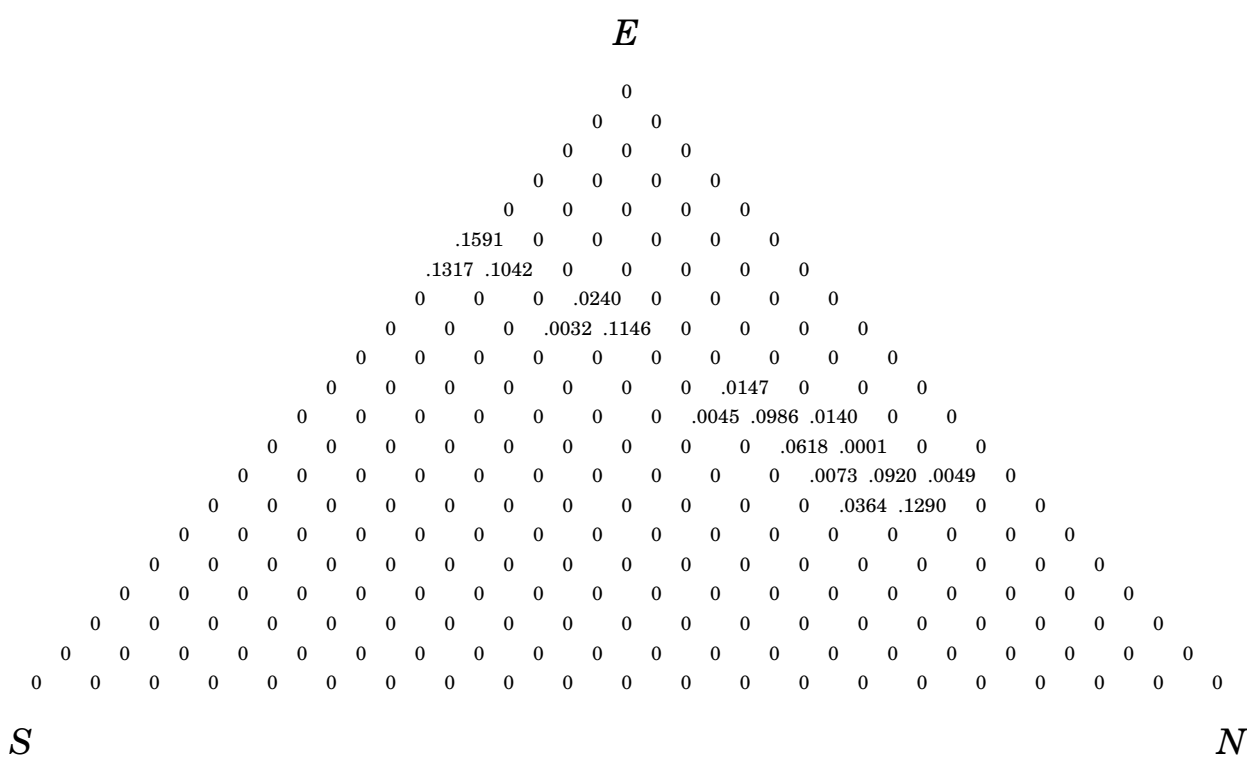

Figure 4.8: Probability distribution over states four years after the introduction of P2P (2003). 


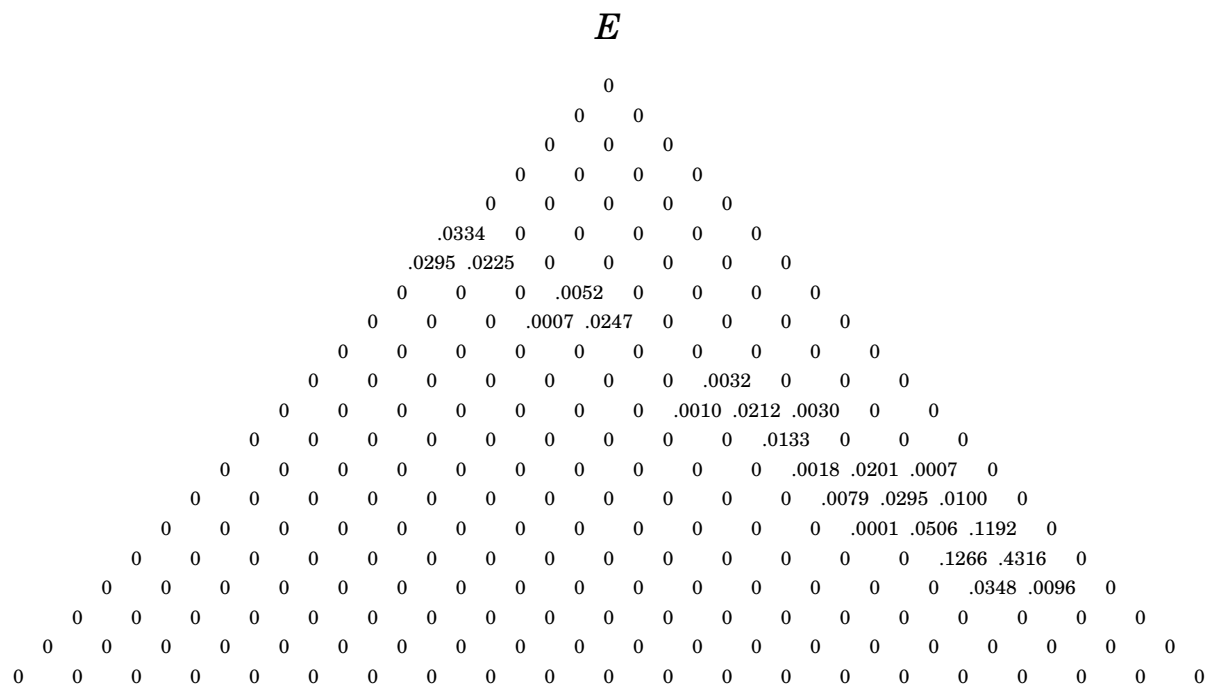

Figure 4.9: Probability distribution over states nine years after the introduction of P2P (2008).

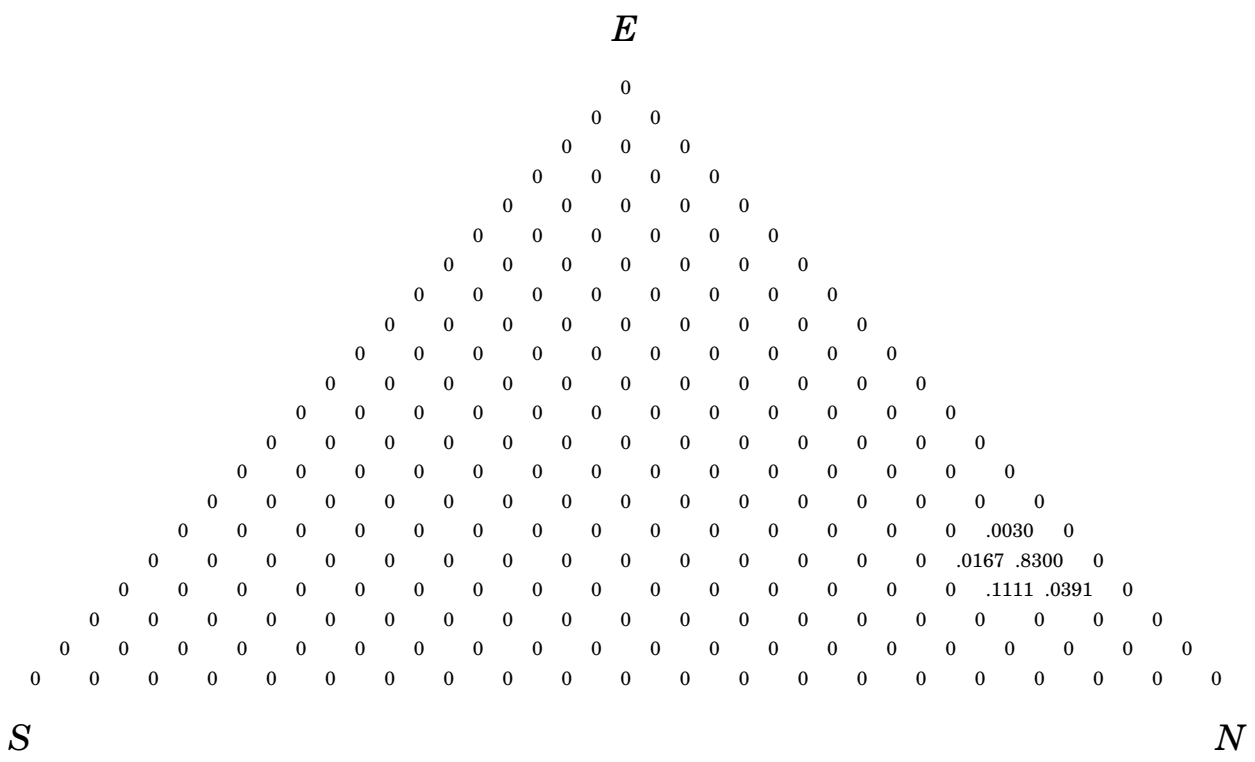

Figure 4.10: Probability distribution over states in steady state after the introduction of P2P (approximately 2020). 



\section{Bibliography}

Arie, G. and P. Grieco (2009). Who pays for switching costs. Working paper: Northwestern University.

Bae, S. and J. Choi (2006). A model of piracy. Information Economics and Policy 18, 303-320.

Belleflamme, P. (2003). Pricing information goods in the presence of copying. The Rate and Direction of Inventive Activity (R Nelson ed.). Princeton University Press.

Blackburn, D. (2004). On-line piracy and recorded music sales. Working Paper: Harvard University.

Blackwell, D. (1962). Discrete dynamic programming. The Annals of Mathematical Statistics 33, 719-726.

Cabral, L. (2009). Small switching costs lead to lower equilibrium prices. Journal of Marketing Research 46, 449-451.

Chen, J., U. Doraszelski, and J. Harrington (2009). Avoiding market dominace: Product compatibility in markets with network effects. Rand Journal of Economics 40, 455-485.

Conner, K. and R. Rumelt (1991). Software piracy: An analysis of protection strategies. Management Science 37, 125-139.

Doraszelski, U. and A. Pakes (2007). A framework for applied dynamic analysis in io. Handbook of Industrial Organization 3. 
Economides, N. (1996). The economics of networks. International Journal of Industrial Organization 14, 673-699.

Farrell, J. and G. Saloner (1985). Standardization, compatibility, and innovation. Rand Journal of Economics 16, 70-83.

Farrell, J. and G. Saloner (1986). Installed base and compatibility: innovation, product preannouncements, and predation. American Economic Review 76, 940-955.

Fudenberg, D. and J. Tirole (2000). Pricing a network good to deter entry. Journal of Industrial Economics 48, 373-390.

Gayer, A. and O. Shy (2003). Internet and peer-to-peer distributions in markets for digital products. Economics Letters 81, 197-203.

Ghosemajumder, S. (2002). Advanced Peer-Based Technology Business Models. MIT Sloan School of Management.

Herings, P., R. Peeters, and M. Yang (2009). Piracy on the internet: Accommodate it or fight it? a dynamic approach. METEOR Research Memorandum RM/09/034, Maastricht University.

Herings, P., R. Peeters, and M. Yang (2010). Competition against peer-topeer networks. Information Economics and Policy, (Forthcoming).

Howard, R. (1960). Dynamic Programming and Markov Processes. Cambridge MA: Technology Press-Wiley.

Jenkins, M., P. Liu, R. Matzkin, and D. McFadden (2004). The browser war: Econometric analysis of markov perfect equilibrium in markets with network effects. Working paper, Stanford University, Stanford.

Johnson, W. (1985). The economics of copying. Journal of Political Economy $93,158-174$.

Judd, K. (1998). Numerical Methods in Economics. Cambridge MA: MIT Press.

Katz, M. and C. Shapiro (1985). Network externalities, competition and compatibility. American Economic Review 75, 424-440.

Katz, M. and C. Shapiro (1986). Technology adoption in the presence of network externalities. Journal of Political Economy 94, 822-841. 
Liebowitz, S. (1985). Copying and indirect appropriability: photocopying of journals. Journal of Political Economy 93, 945-957.

Liebowitz, S. (2004). Will mp3 downloads annihilate the record industry? the evidence so far. Advances in the Study of Entrepeneurship, Innovation, and Economic Growth 15, 229-260.

Liebowitz, S. (2006). File sharing: Creative destruction or just plain destruction? Journal of Law and Economics 49, 1-28.

Markovich, S. (2008). Snowball: A dynamic oligopoly model with indirect network externalities. Journal of Economic Dynamics \& Control 32, 909-938.

Markovich, S. and J. Moenius (2009). Winning while losing: Competition dynamics in the presence of indirect network effects. International Journal of Industrial Organization 27, 346-357.

Nocke, V., M. Peitz, and K. Stahl (2007). Platform ownership. Journal of the European Economic Association 5, 1130-1160.

Novos, I. and M. Waldman (1984). The effects of increased copyright protection: an analytical approach. Journal of Political Economy 92, 236246.

Oberholzer-Gee, F. and K. Strumpf (2007). The effect of file sharing on record sales: An empirical analysis. Journal of Political Economy 115, $1-42$.

Peitz, M. and P. Waelbroeck (2004). The effect of internet piracy on cd sales: Cross-section evidence. Review of Economic Research on Copyright Issues 1, 71-79.

Peitz, M. and P. Waelbroeck (2005). An economist's guide to digital music. CESifo Economic Studies 51, 359-428.

Peitz, M. and P. Waelbroeck (2006a). Piracy of digital products: A critical review of the theoretical literature. Information Economics and Policy 18, 449-476.

Peitz, M. and P. Waelbroeck (2006b). Why the music industry may gain from free downloading - the role of sampling. International Journal of Industrial Organization 24, 907-913. 
RIAA (2007). The cd: A better value than ever. An RIAA report prepared by the Communications and Strategic Analysis Department Of the Recording Industry Association of America.

RIAA (2009). 2008 year-end shipment statistics.

Rob, R. and J. Waldfogel (2006). Piracy on the high c's: Music downloading, sales displacement, and social welfare in a sample of college students. Journal of Law and Economics 49, 29-62.

Takeyama, L. (1994). The welfare implications of unauthorized reproduction of intellectual property in the presence of network externalities. Journal of Industrial Economics 42, 155-166.

Yang, M. (2010). Markets of information goods facing a strong p2p network. Working paper, Maastricht University.

Zentner, A. (2006). Measuring the effect of file sharing on music purchases. Journal of Law and Economics 49, 63-90. 


\section{Nederlandse samenvatting (Dutch summary)}

Dit proefschrift behandelt enkele complexe kwesties omtrent de online piraterij van digitale goederen in het eerste decennium van de 21 ste eeuw. We bekijken het strategisch gedrag van de producent van de digitale goederen, een monopolist, die de bedreiging van online piraterij onder ogen ziet. "Hoe bepaalt de producent de optimale prijs voor zijn producten?" We bestuderen de economische effecten van online piraterij op zowel de consumenten als de producent. "Wie zijn de winnaars en de verliezers, en in welke mate?" We analyseren de effecten van de handhaving van de wet betreffende intellectuele eigendomsrechten. "Is het achtervolgen en het straffen van de downloaders bevordelijk voor het maatschappelijk welzijn?" We onderzoeken voorwaarden die noodzakelijk zijn voor de markt van digitale goederen om bepaalde kenmerken te laten zien. "Wanneer wordt de markt gediend door enige of veelvoudige actieve platforms, en wanneer wordt de markt volledig of gedeeltelijk gediend?" Tot slot bestuderen we de dynamische ontwikkeling van de concurrentie tussen de digitale goederenproducent en het plagiërende platform, vanaf het allereerste begin, en het effect daarvan op de consumenten, de producent en de maatschappij als geheel.

Hoofdstuk 2 is een aangepaste versie van artikel (Herings, Peeters, and Yang 2010), dat markten met een van nature zwak P2P netwerk onderzoekt. Hierbij gebruiken we een parameterveronderstelling waarbij indien een netwerk leeg is, niemand zal toetreden. Hierdoor is het netwerk kwets- 
baar voor een mislukte coördinatie. In het bijzonder, wanneer alle consumenten verwachten dat het netwerk zich niet zal vormen, zal het voor geen enkele consument individueel rationeel zijn zich bij het netwerk aan te sluiten, ondanks het feit dat het voor sommigen beter zou zijn geweest als zij zich allemaal bij het netwerk zouden hebben aangesloten.

We modelleren de interactie tussen de producent en het P2P netwerk als spel met twee stadia. We analyseren het model door zijn zogenaamde subgame-perfecte evenwichten te bestuderen. We verstrekken een volledige karakterisering van de subgame-perfecte evenwichten van het model en beperken onze aandacht tot het subgame-perfecte evenwicht waar het grootst mogelijke netwerk zich vormt. We typeren de evenwichtsmarktstructuren, en analyseren voor elke structuur hoe de winst voor de producent, het consumentensurplus en het totale welzijn worden beïnvloed door verschillende parameters zoals de kostenfactor van het downloaden en de verscheidenheid van voorkeuren van de consumenten.

Er worden gevonden drie mogelijke evenwichtmarktstructuren. Allereerst, kan de producent als monopolist fungeren, die, afhankelijk van de omstandigheden, of gedeeltelijk of volledig de markt dient. In dit geval, vormt het P2P netwerk zich niet. Hiervoor moet de intrinsieke waarde van de fysieke vorm van het product wezenlijk hoger zijn dan dat van de digitale vorm. Ten tweede kan de producent het vormen van het netwerk verhinderen door middel van een netwerk-afschrikkend prijsbeleid. Wederom zal de producent de markt gedeeltelijk of volledig dienen. Tot slot kunnen de producent en het $\mathrm{P} 2 \mathrm{P}$ netwerk naast elkaar bestaan. In dit geval wordt gegarandeerd dat de markt volledig zal worden gediend.

Door middel van comparatief-statische analyses, vinden we dat hoe groter de kostenfactor van het downloaden is, des te minder waarschijnlijk het is dat een P2P netwerk zich vormt, en des te hoger de winst van de producent zal zijn. Indien de twee platforms naast elkaar bestaan, bestaat er opvallend genoeg een negatieve relatie tussen de kostenfactor en de prijs die door de producent wordt bepaald. Dit is een interessante vorm van platformconcurrentie. In ons model verminderen downloadkosten onevenredig met de netwerkgrootte. De eerste consumenten die zich bij het netwerk aansluiten zijn essentieël voor het bepalen van de downloadkosten. Indien de grootte van het evenwichtsnetwerk vrij klein (inherent zwak) is heeft de producent 
een aansporing nodig om "hard" te spelen door middel van het zetten van een lage prijs. Omgekeerd, als de kostenfactor klein is, is het evenwichtsnetwerk groot, en zal het netwerk zich gemakkelijker vormen. In dat geval kan de producent zich beter terugtrekken uit de prijscompetitie en een hoge prijs rekenen om de meeste winst te halen uit de klanten die de fysieke vorm van het product willen kopen. De evenwichtswinst is stijgend met de kostenfactor, ondanks de dalende prijs.

In zowel de gedeeltelijk-gediende monopolistische markt met netwerkafschrikkende tarifering als de volledig-gediende markt met multi-platform co-existentie, vermindert het totale welzijn in de kostenfactor. Dit impliceert dat het welzijn vermindert, indien de autoriteiten de bescherming van intellectuele eigendomsrechten te sterk benadrukken.

Hoofdstuk 3 is een aangepaste versie van het artikel (Yang 2010), dat markten met een inherent sterk P2P netwerk bestudeert. In dit hoofdstuk wordt verondersteld dat er minstens één consument bestaat die zich bij het P2P netwerk zal aansluiten zelfs wanneer het netwerk leeg zou moeten zijn. Deze aanname is gebaseerd op de situatie waar er altijd een aantal fanatieke P2P verdedigers zijn die het netwerk levend houden door te uploaden.

Daarbij nemen we afstand van de veronderstelling dat de verscheidenheid van voorkeuren van de consumenten klein is. Specifieker, consumenten met extreme voorkeuren zullen niet de minst geprefereerde versie van het product gebruiken, zelfs als het kostenloos is. Zodoende, kunnen we het scenario beschrijven waar de markt zowel door de producent als het netwerk wordt gediend.

Onze bevinding is dat de mislukte coördinatie bij het vormen van netwerken geen probleem is zodra we een hoog niveau van verscheidenheid aan voorkeuren toestaan binnen het model. We vinden in totaal vijf mogelijke evenwichtsmarktstructuren in dit hoofdstuk. De meest geobserveerde marktstructuur is die waarin de producent in prijs concurreert. De winst van de producent lijdt onder het bestaan van P2P, terwijl het totale welzijn hiervan profiteert.

De producent kan zorgen dat het netwerk zich niet vormt, indien de verscheidenheid van voorkeuren laag is, de kwaliteit van de fysieke vorm hoog is, en de wettelijke handhaving strikt is. De producent kan ook de hele markt willen dienen, wanneer de waarde van zijn product beduidend hoger 
is dan die van de plagiaat versie en de verscheidenheid van voorkeuren laag is. Een andere speciale marktstructuur is die waarbij de markt gedeeld wordt. Deze marktstructuur ontstaat wanneer de verscheidenheid van voorkeuren zo is dat de producent kiest om precies die consumenten te dienen die niet willen downloaden. De producent laat het grootst mogelijke netwerk onstaan, en dient dan de overblijvende consumenten. De producent en het netwerk dienen samen de markt volledig, zonder dat ze met elkaar concurreren. Tevens is er een mogelijkheid waar de producent het netwerk kan negeren en als monopolist handelt. Dit is het geval wanneer de verscheidenheid van voorkeuren erg groot is. Echter in dit scenario vermindert zowel de winst van de producent als het consumentensurplus in de verscheidenheid van voorkeuren. Als gevolg daalt het totale welzijn. Dit resultaat bevestigt de bevindingen in hoofdstuk 2 .

Hoofdstuk 4 is een aangepaste versie van het artikel (Herings, Peeters, and Yang 2009), dat gebruik maakt van een dynamisch stochastisch model om het optimale prijsbeleid van de bedrijven van de muziekopname te bepalen, in de situatie waar P2P netwerken hun CD verkoop ondermijnen. Een voordeel van deze methode is dat we kunnen bestuderen hoe de netwerken zich ontwikkelen in een dynamisch proces, zonder enige veronderstelling te hoeven maken betreffende de coordinatie tussen consumenten zoals in de conventionele statische modellen in hoofdstuk 2 en 3. We kunnen ook precies waarnemen hoe de producent zijn prijs bepaalt, namelijk afhankelijk van zijn marktaandeel en het marktaandeel van het netwerk. Soms stelt de producent een zeer lage prijs vast om cruciale marktaandelen te winnen om zo tegen het P2P netwerk te vechten. Tegelijkertijd bestaan er situaties waar de producent hoge prijzen vaststelt, en daardoor het netwerk toelaat, om zo een zo hoog mogelijke winst uit zijn klanten te kunnen behalen. Naast het gebruiken van het model om te beschrijven wat in de CD-markt gebeurd zou kunnen zijn sinds 1999 toen P2P werd geïntroduceerd, kunnen we het model ook gebruiken om toekomstige verkoophoeveelheden, en hierdoor ook veranderingen in het consumentensurplus en het totale welzijn, te voorspellen. Het model toont bijvoorbeeld aan dat de verkoophoeveelheid van CD's van het jaar 2008 in de Verenigde Staten 374.3 miljoen exemplaren zou moeten zijn, terwijl het echte cijfer 384.7 miljoen is. Het voorspelt ook dat rond het jaar 2020, het jaar waarin volgens de schattingen van het 
model het lange termijn evenwicht bereikt zou moeten zijn, de CD verkoop ongeveer tot 231.2 miljoen exemplaren per jaar gedaald zal zijn. Bovendien, kan men met dit model de groei in het sociale welzijn schatten die in de afgelopen jaren toegekend kon worden aan de P2P netwerken. In 2003, vier jaar nadat $\mathrm{P} 2 \mathrm{P}$ werd geïntroduceerd, daalde de totale jaarlijke winst in de muziekindustrie met $\$ 1.6$ miljard, terwijl het totale welzijn met $\$ 13.1$ miljard steeg. In 2008 is de jaarlijkse industriewinst verder gedaald met $\$ 1.4$ miljard, en steeg het welzijn met $\$ 12.4$ miljard. In vergelijking met 1999 , is dit dus $\$ 25.6$ miljard meer. In het lange-termijn evenwicht (rond 2020 ), zal de jaarlijkse industriewinst slechts $\$ 865.5$ miljoen bedragen, en zal het totale welzijn met $\$ 29.7$ miljard gestegen zijn. We zijn ons er sterk van bewust dat deze ramingen niet erg nauwkeurig zullen zijn, aangezien de marktvoorwaarden voor een alsmaar veranderende industrie gezien de snel veranderende tijden onmogelijk te voorspellen zijn. Maar dit onderzoek geeft een grove indicatie van wat er in de toekomst zou gebeuren indien alles onveranderd blijft.

In de vergelijkende statische analyse van het model, is één van onze belangrijkste bevindingen dat er een negatieve relatie bestaat tussen het totale welzijn en de kostenfactor van het downloaden. Dit resultaat bevestigt de bevindingen van artikelen zoals (Rob and Waldfogel 2006) en ook hoofdstuk 2 en 3 uit dit proefschrift, dat het bestaan van P2P het totaal welzijn verbetert. Dit suggereert dat door strikte intellectuele eigendomsrechten af te dwingen en het downloaden voor consumenten moeilijker te maken, de overheid de maatschappij impliciet verhindert een hoger welzijnsniveau te bereiken. 



\section{Curriculum Vitae}

Michael Shuo Yang was born on 3 October 1979, in Changsha, China, where he received his high school diploma from ChangJun High School in 1998. Later that year, he started his higher education in European Business Administration at Hogeschool Holland in Diemen, the Netherlands. Upon receiving his propaedeutic certificate in 1999, he switched to study Economics at Maastricht University where he received his Master's degree in Economics majored in Infonomics in 2005. After carrying out half a year of full-time teaching position at the faculty, he received a research grant for a $\mathrm{PhD}$ position in the field of Industrial Organization, Microeconomics, at the department of Economics. The results thereof are presented in this dissertation. After the completion of the $\mathrm{PhD}$, he accepted a position at Frontier Economics, an economic consultancy based in London, starting in December 2010 . 
